\title{
PRENATAL EXPOSURE TO ORGANOPHOSPHORUS PESTICIDES AND CHILDHOOD NEURODEVELOPMENTAL PHENOTYPES
}

\author{
Melissa A. Furlong
}

A dissertation submitted to the faculty at the University of North Carolina at Chapel Hill in partial fulfillment of the requirements for the degree of Doctor of Philosophy in the Department of Epidemiology in the Gillings School of Global Public Health.

Chapel Hill

2016

Approved by:

Stephanie M. Engel

Amy H. Herring

Julie L. Daniels

Larry Engel

Barbara Goldman 
(C) 2016

Melissa A. Furlong

ALL RIGHTS RESERVED 


\begin{abstract}
Melissa A. Furlong: Prenatal Exposure to Organophosphorus Pesticides and Childhood Neurodevelopmental Phenotypes

(Under the direction of Stephanie M. Engel)

Background: Neurodevelopmental traits are often treated as independent in etiological
\end{abstract} studies, although neurological functions exhibit complex correlational structures. Allowing traits to cluster into phenotypes may produce outcomes that are more clinically and biologically relevant. Additionally, environmental exposures often occur in a mixture. Accounting for such a possibility may reduce bias that results from assuming exposures are independent. We sought to estimate associations between organophosphorus pesticides and neurodevelopment after accounting for these possibilities.

Methods: A prospective birth cohort of 404 mother/infant pairs were followed from pregnancy until the children were 6 and 7-9 years of age. Factor analysis was performed on parent-report measures of child executive functioning and behavior, and performance-based measures of IQ. We estimated associations between demographic characteristics, maternal characteristics and environmental exposures during pregnancy and early childhood, and the neurodevelopmental factor scores after accounting for correlations among the factors.

Results: We determined the existence of a seven factor solution. Smoking during pregnancy, canned fish consumption, maternal education, and HOME environment were associated with various factors. Prenatal exposure to $\sum$ DMPs was associated with worse Internalizing factor scores but better Executive Functioning factor scores, while $\sum$ DEPs were associated with worse Working Memory Index scores. Estimates were generally stronger for factor scores than for individual instrument-specific items. 
Conclusions: Associations between prenatal exposure to OPs and worse internalizing behaviors and working memory scores are supported by prior findings in both human and animal studies. Associations with improved executive functioning are not supported by prior literature and may be a result of residual confounding by maternal executive functioning and dietary sources of OPs.

A phenotypic outcome modeling approach may provide advantages over more traditional outcome modeling approaches. 


\section{ACKNOWLEDGEMENTS}

I have the most inspiring mentor who provides amazing guidance and settles for nothing less than my best effort. Stephanie Engel is an extraordinarily talented and caring advisor and has imparted wisdom in so many different aspects of life, both academic and personal. I could not have done even a tenth of this dissertation without her mentorship. My committee has also been invaluable and consistently available to answer questions and offer guidance. The quality of instruction, research, and resources at the University of North Carolina has been excellent and I must acknowledge the institution for enabling my work, and the department of Epidemiology for the world-class instruction in epidemiology. l'd also like to acknowledge the NIEHS T32 training grant for funding me and allowing me to focus on research without the additional strain of a research assistantship. There are no words to describe how grateful I am to my extraordinary husband, Evan MacLean, for being not only a role model but also the most supportive partner I could ever hope for. And finally, I have to acknowledge my daughter Madeleine, for teaching me the invaluable lesson of functioning on broken and limited sleep, giving me firsthand experience in childhood development, and making my life complete. 


\section{TABLE OF CONTENTS}

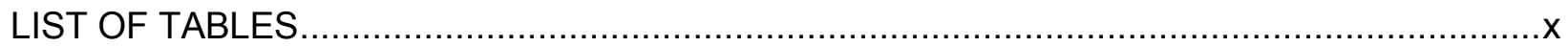

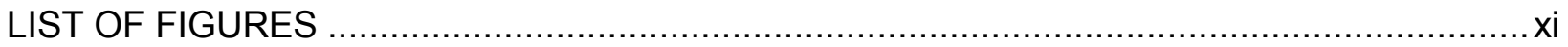

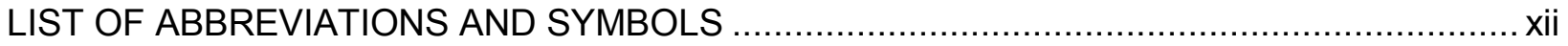

CHAPTER I. INTRODUCTION AND SPECIFIC AIMS .................................................... 1

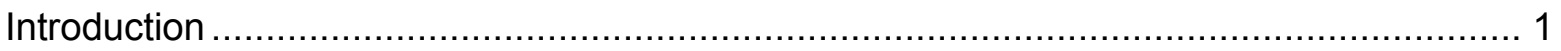

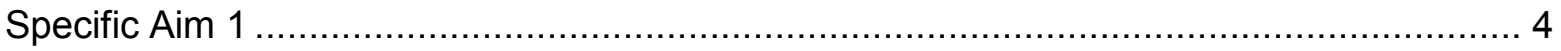

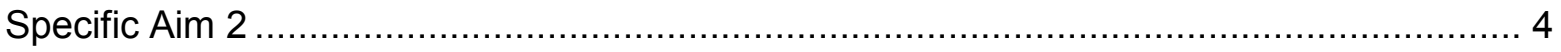

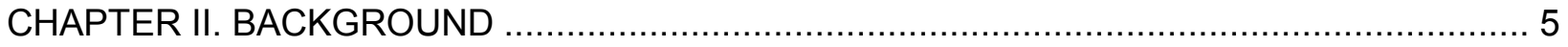

Source, Route, and Estimation of Organophosphorus Pesticide Exposure ........................ 5

Organophosphorus Pesticide Use in the United States .......................................... 5

Environmental Fate, Breakdown, and Metabolism of Ops …….............................. 5

Source of Exposure

Associations between Prenatal Exposure to Organophosphorus Pesticides and

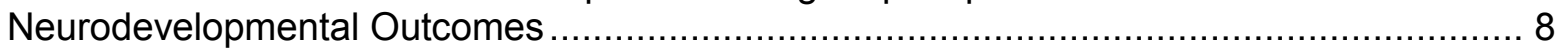

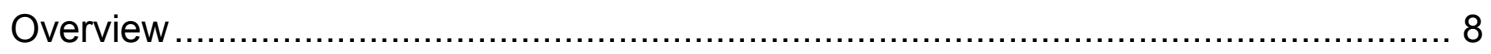

OPs and Neurodevelopment: Birth Cohorts.........................................................10

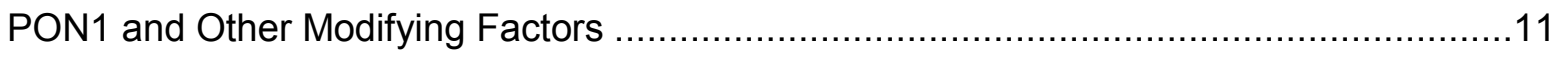

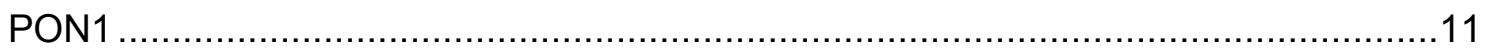

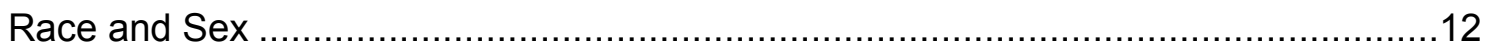

Other Xenobiotics and Neurodevelopmental Outcomes ........................................13

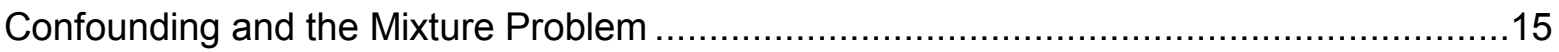

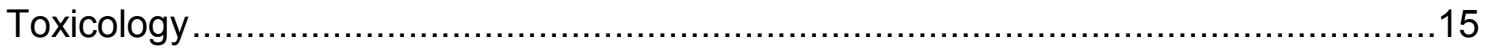

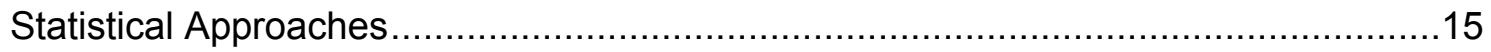




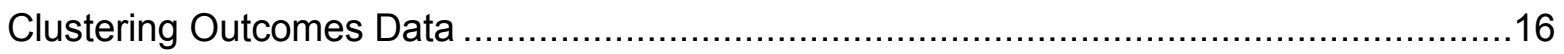

Advantages of Clustering Outcomes Data: Clinically Relevant Outcomes ...................17

Advantages of Clustering Outcomes Data: Relevance to Biological

Interpretations of OP Mechanisms .................................................................18

Advantages of Clustering Outcomes Data: Spotlight on ADHD ...............................19

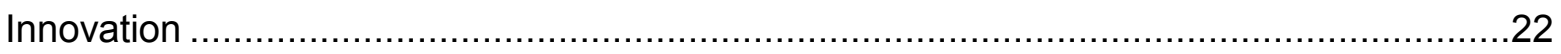

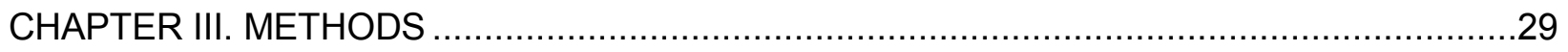

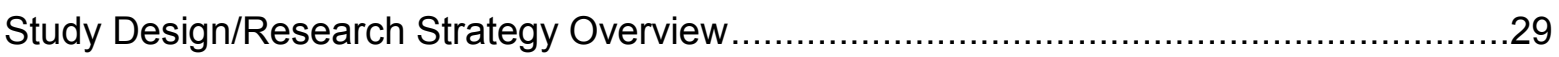

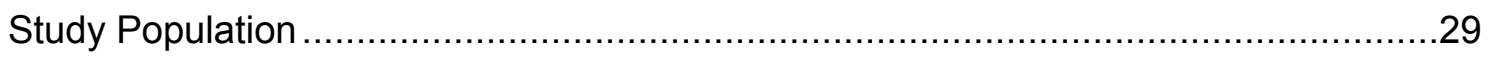

Exposure, Outcome, and Covariate Assessment ……....................................................

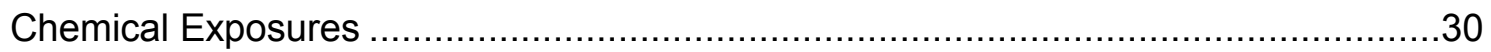

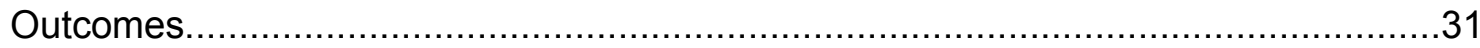

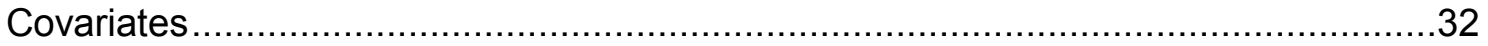

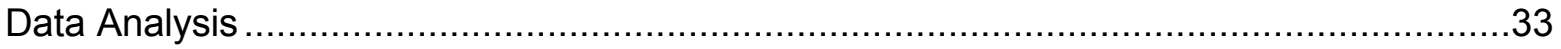

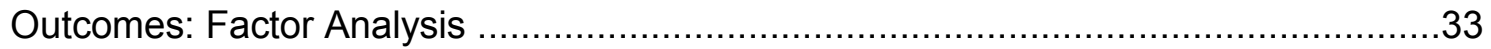

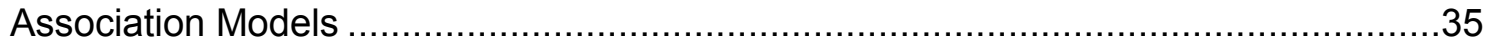

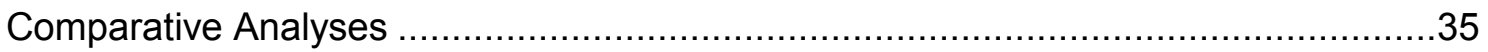

CHAPTER IV. EARLY LIFE CHARACTERISTICS AND NEURODEVELOPMENTAL PHENOTYPES IN THE MOUNT SINAI CHILDREN'S ENVIRONMENTAL

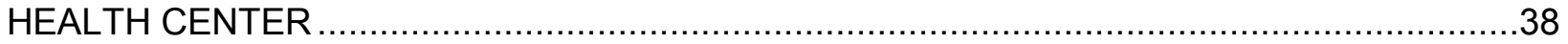

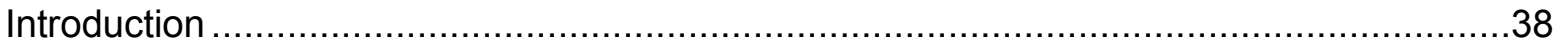

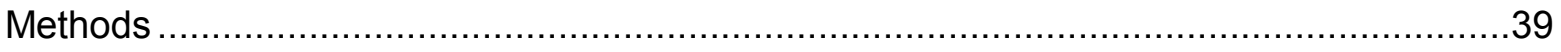

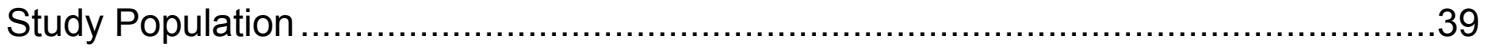

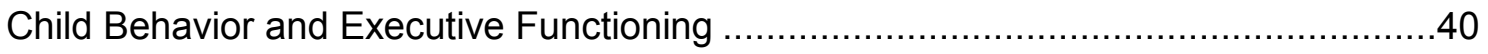

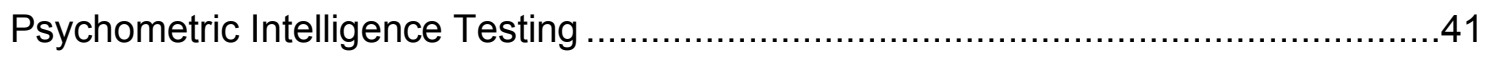

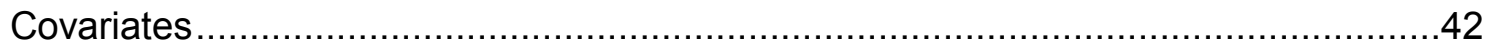

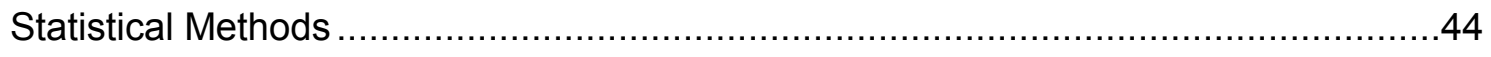

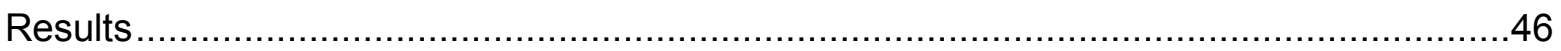


Study Population Characteristics and Follow-up..................................................

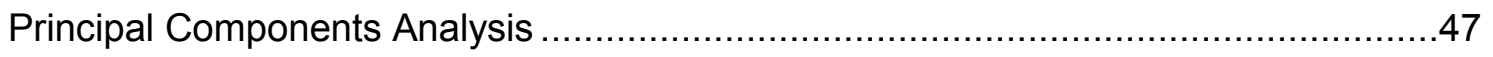

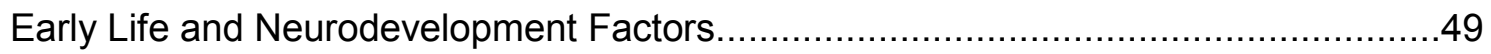

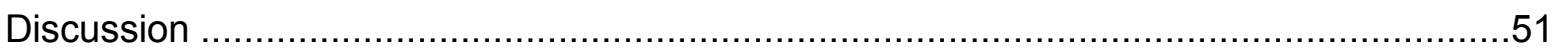

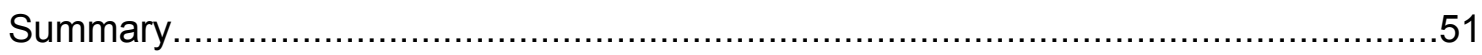

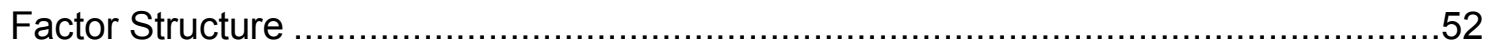

Early Life Characteristics and Neurodevelopmental Factors ...................................53

Maternal Prenatal Smoking and Neurodevelopment .............................................54

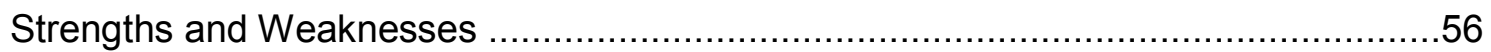

CHAPTER V. ASSOCIATIONS BETWEEN PRENATAL EXPOSURE TO

ORGANOPHOSPHORUS PESTICIDES AND CHILDHOOD

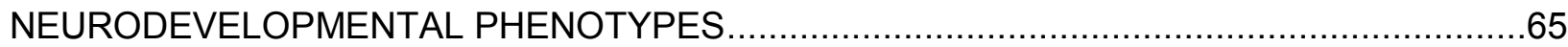

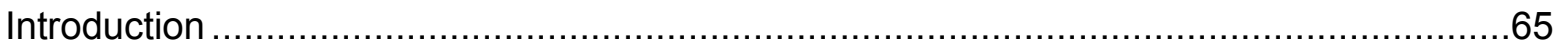

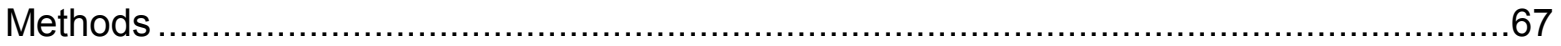

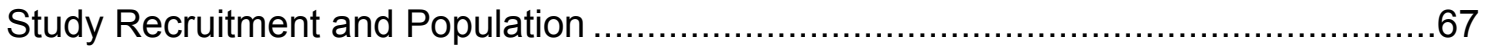

Exposure Biomarker Measurements ………...................................................67

Child Behavior, Executive Functioning, Psychometric Intelligence Testing ..................68

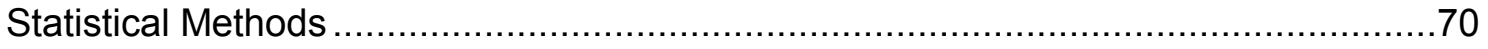

Neurodevelopmental Factor Analysis and Outcomes ….........................................70

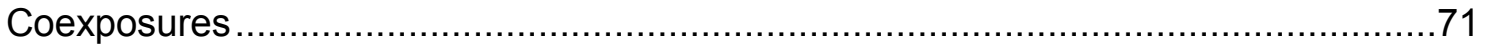

Bayesian Exposure Mixture Analyses …………..................................................71

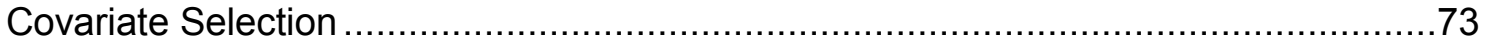

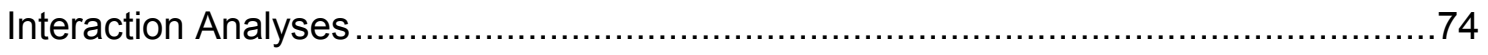

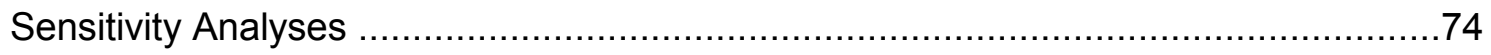

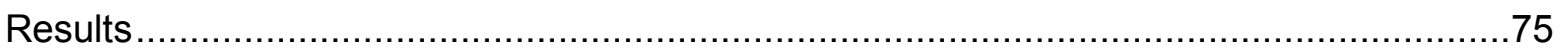

Bayesian Exposure Mixture Modeling of Neurodevelopmental Factors .......................76

Heterogeneity in Associations by Race .............................................................. 


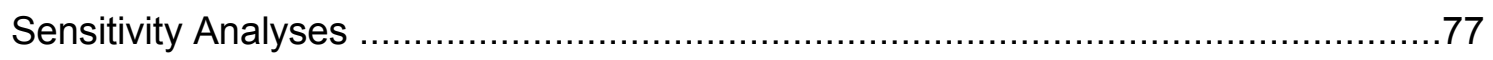

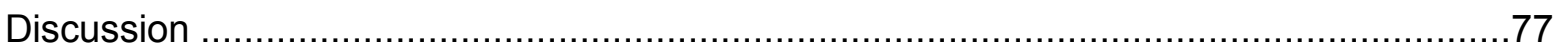

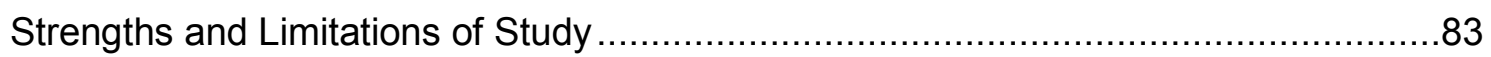

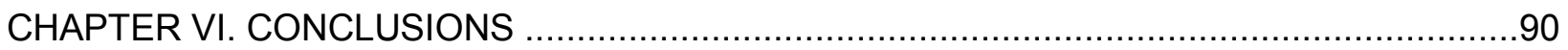

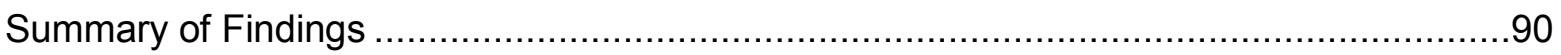

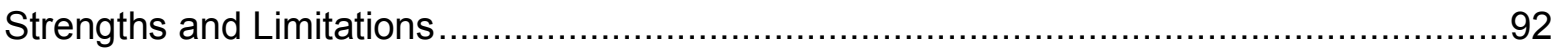

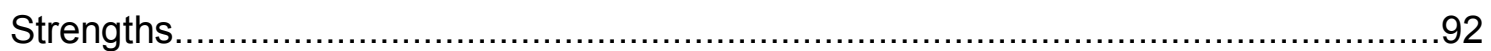

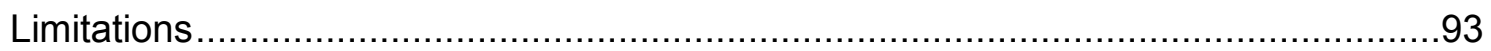

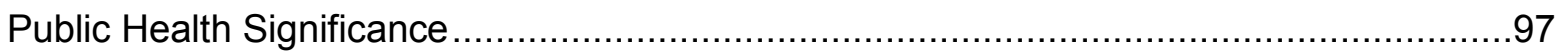

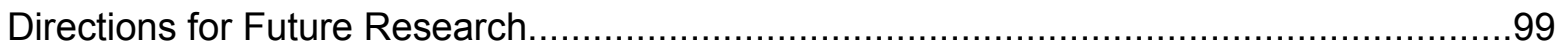

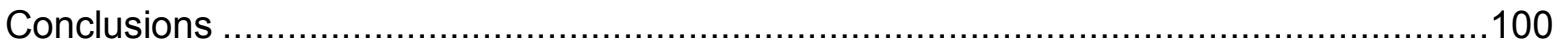

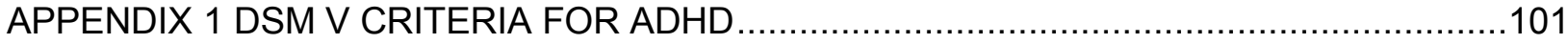

APPENDIX 2 PROMAX ROTATED FACTOR PATTERN STRUCTURE AND

ITEM LOADINGS OF CHILDHOOD NEURODEVELOPMENTAL SCALES

$(\mathrm{N}=210)$.

APPENDIX 3 ASSOCIATIONS BETWEEN SMOKING AND

NEURODEVELOPMENTAL OUTCOMES, BY OUTCOME MEASUREMENT

METHOD

APPENDIX 4 BIVARIATE ASSOCIATIONS BETWEEN EARLY LIFE

CHARACTERISTICS AND NEURODEVELOPMENTAL FACTORS ${ }^{1}$ IN THE

MOUNT SINAI CHILDREN'S ENVIRONMENTAL HEALTH CENTER

APPENDIX 5 FREQUENTIST ASSOCIATIONS BETWEEN OPS AND

NEURODEVELOPMENTAL FACTOR SCORES, WITH AND WITHOUT

ADJUSTMENT FOR CO-EXPOSURES

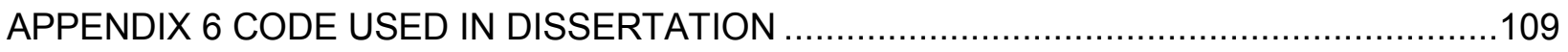

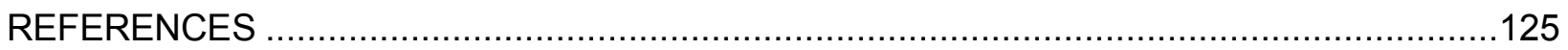




\section{LIST OF TABLES}

Table 1 Common Metabolites of Selected Organophosphorus Pesticides

Table 2 Summary of Prenatal Exposure to OPs and Associations with

Neurodevelopment in Children's Environmental Health Center Cohorts ...

Table 3 Characteristics of Study Population at Enrollment and Follow Up, Mount

Sinai Children's Environmental Health Cohort....

Table 4 Instruments Included in Factor Analysis of Behavior, Executive

Functioning, and IQ.....

Table 5 Varimax Rotated Factor Pattern Structure and Item Loadings of Childhood

Neurodevelopmental Scales $(n=210)$.

Table 6 Covariate-Adjusted Linear Regression Associations Between Prenatal and Early Life Characteristics and Neurodevelopmental Factors In The Mount Sinai Children's Environmental Health Cohort $(n=162)$

Table 7 Comparison of Prenatal Smoking and Neurodevelopment Associations by Analysis Method $(n=162)$.

Table 8 Characteristics of Mount Sinai Children's Environmental Health Center Study Population by Follow Up Status, For Those with Complete Panel of Biomarkers.

Table 9 Distributions of Organophosphorus Pesticide Metabolites and

Co-exposures, Among Participants with Complete Follow-Up Measurements

Table 10 Overall Associations between OPs and Neurodevelopmental Factor

Scores $(n=141)$

Table 11 Associations between DMPs and Individual Factor Items from Executive

Functioning and Internalizing Factors $(n=141)$ 


\section{LIST OF FIGURES}

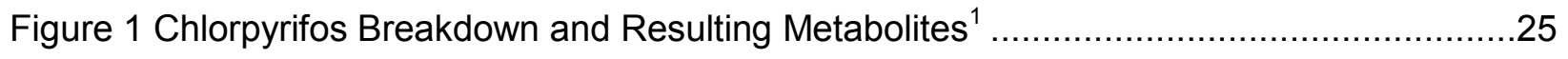

Figure 2 Origination and Follow-up of Participants Included in Factor Analysis and

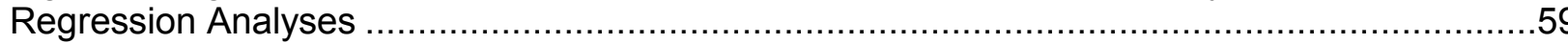




\section{LIST OF ABBREVIATIONS AND SYMBOLS}

ASD Autism Spectrum Disorder

ADHD Attention Deficit Hyperactivity Disorder

BASC Behavioral Assessment System for Children

BSID-II Bayley Scales for Infant Development $2^{\text {nd }}$ edition

CBCL Child Behavior Checklist

BRIEF Behavioral Rating Inventory of Executive Functioning

DAPs Dialkylphosphates

¿DEPs Summed Diethylphosphates

¿DMPs Summed Dimethylphosphates

FA Factor Analysis

EF Executive Functioning

IQ Intelligence Quotient

LOD Limit of Detection

MCMC Markov Chain Monte Carlo

MDI Mental Development Index

PDI Psychomotor Development Index

OCD Obsessive Compulsive Disorder

ODD Oppositional Defiant Disorder

OPs Organophosphorus Pesticides

PDD Pervasive Developmental Disorder

WISC Wechsler Intelligence Scale for Children

WPPSI Wechsler Preschool and Primary Scales of Intelligence 


\section{CHAPTER I. INTRODUCTION AND SPECIFIC AIMS}

\section{Introduction}

Prenatal exposure to organophosphorus pesticides has been associated with a wide range of neurodevelopmental outcomes at different stages of childhood, including deficits in childhood cognition (Bouchard et al., 2011a; S. M. Engel et al., 2015; S. Engel et al., 2011; V. Rauh et al., 2011), social responsiveness (Furlong et al. 2014), and behavior (Marks et al., 2010). The affected domains are either hallmarks of, or are highly associated with, AttentionDeficit Hyperactivity Disorder (ADHD) (Willoughby 2003), a syndrome characterized by inattention, hyperactivity, and impulsive behaviors, suggesting a plausible association between OPs and this overarching disorder. However, epidemiological studies that have considered prenatal exposures and childhood neurodevelopment or ADHD behaviors generally model exposures and outcomes in a 1:1 analytic framework (i.e., one chemical metabolite and one neurodevelopmental scale). There are several limitations to this approach.

Using exposure mixture models can account for confounding by correlated contaminants. Women are actually exposed to complex mixtures of pollutants, and modeling exposures as though they are independent may result in biased estimates. Chemicals may be correlated with each other due to similar sources, such as plasticizers in products or insecticides for pest control (Engel and Wolff 2013). Interestingly, toxicology studies have begun to show that chemicals that initially act via different biological pathways may still result in similar downstream neuronal outcomes (such as increased neurotoxicity or altered neuronal differentiation) and may thus be important components in considering biological interactions or confounding with other chemicals. 
Examining neurological scales and subscales singly is an overly simplistic representation of a complex system. Neurological development and functioning is a complex, interdependent system. Scientists have compartmentalized neurological functioning into bins (i.e., working memory, inhibitory control, perceptual reasoning, aggression), although the human brain evolved as an interdependent system. There are two major advantages in consolidating outcome data and considering phenotypes of neurodevelopment rather than using models that assume independence of each neurodevelopmental outcome. First, examining neurodevelopmental outcomes in the context of other impairments is clinically relevant. Several neurological functions rely on each other (for instance, the "higher-level" inhibitory control relies on more "basic" processing speed capability (Ridderinkhof and van der Molen 1997)), and deficits in one area are oftentimes compensated for by other areas. For instance, a deficit in working memory alone was only associated with poor social skills when accompanied by deficits in attention, hyperactivity, and impulsivity (Kofler et al. 2011), and working memory may only be associated with poorer academic achievement when accompanied by symptoms of inattention (Rogers et al. 2011). Second, patterns of correlations across scales may also point to different clinical phenotypes - when aggression problems are accompanied by high impulsivity, researchers note a different profile of aggressive behavior (reactive-impulsive) than when aggression is accompanied by higher levels of self-control (controlled-instrumental) (Vitiello and Stoff 1997), and these differences are associated with different patterns of behaviors and criminality (Nelson and Trainor 2007). Therefore, examining performance on scales within the context of other instruments can provide clinically relevant profiles of neurodevelopment that are also differentially associated with later life outcomes.

Clustering behavioral and cognitive performance across scales can create factors that reflect common etiological mechanisms and allow a more biologically relevant consideration of the OP-neurodevelopment relationship. Behavioral and cognitive deficits that are clinically and statistically correlated may reflect a common etiological insult. For instance, OPs influence 
serotonergic processing (Aldridge et al. 2005b; Aldridge et al. 2004; Slotkin et al. 2008b; Slotkin and Seidler 2005). Serotonin, in turn, can influence aggression, social behaviors, depression, and ADHD (Cadoret et al. 2003; Eley et al. 2004; Zoroğlu et al. 2002), and these symptoms may cluster in response to a global disruption in upstream processing like serotonergic functioning. Animal and human studies do support that OPs may be associated with those behavioral outcomes (Bouchard et al. 2010; Eskenazi et al. 2007; Furlong et al. 2014; Middlemore-Risher et al. 2010; Ricceri et al. 2003; Ricceri et al. 2006), which are perhaps mediated by serotonergic functioning (Slotkin and Seidler 2008; Venerosi et al. 2010). However, aggression is also influenced by non-serotonergic mechanisms, including disruptions to the frontal and prefrontal cortex, GABA, oxytocin, dopamine, and MAO-A systems (reviewed in (Nelson and Trainor 2007)). OPs do not necessarily influence all of these systems, and patterns of behaviors that are influenced by serotonin are not associated with all of these systems either. However, when outcomes such as aggression are analyzed individually outside of the context of other symptoms, aggression from any neurological source is lumped, including aggression that is the result of non-OP-influenced mechanisms. By instead considering behavioral and cognitive clusters, we may characterize patterns of development (such as ADHD (Castellanos et al. 2006; Mattison and Mayes 2010; Semrud-Clikeman et al. 2010; Sinzig et al. 2008)) that theoretically represent common etiologies.

Using data from the prospective Mount Sinai Children's Environmental Health Center cohort, we take advantage of multi-pollutant exposure and multidimensional and longitudinal neurodevelopmental outcome data to create a model that: 1) is a more accurate depiction of real-life exposure mixtures, and 2) takes into consideration the interdependency of neurodevelopment to create behavioral and cognitive profiles. This model will account for confounding by co-contaminants and take advantage of clustering across scales to create neurodevelopmental profiles that may be more representative of biological pathways affected by OPs than the $1: 1$ analytic approach. 


\section{Specific Aim 1}

To create phenotypes of neurodevelopment, using measures of cognition (Wechsler Preschool and Primary Scale for Infants and Toddlers [WPPSI], Wechsler Intelligence Scale for Children-IV [WISC-IV]), behavior (Behavioral Assessment System for Children [BASC]), and executive functioning (Behavioral Rating Inventory of Executive Functioning [BRIEF]), in the Mount Sinai Children's Environmental Health Center Cohort.

Aim 1.1: To create phenotypes using a principal components analysis with orthogonal varimax rotation.

Aim 1.2: To estimate associations between prenatal and early life characteristics and neurodevelopmental factors created in Aim 1.1.

Aim 1.3: To compare estimates of associations between prenatal smoking and neurodevelopment using 1) factors derived from an orthogonal rotation, 2) factors derived from an oblique rotation, and 3) instrument-specific composite scores.

\section{Specific Aim 2}

To estimate associations of OPs with phenotypes of neurodevelopment in a mixture model, and assess modification by potentially important characteristics.

Aim 2.1: Estimate associations between OPs and neurodevelopmental phenotypes in a mixture model that accounts for possible confounding by other classes of xenobiotic chemicals (phthalates, phenols, and pyrethroids) in a Bayesian framework.

Aim 2.2: Assess modification of the OP-neurodevelopment relationship by race, sex, and paraoxonase $1(P O N 1)$ genotype, a gene integral to the metabolism of organophosphorus pesticides. 


\section{CHAPTER II. BACKGROUND}

\section{Source, Route, and Estimation of Organophosphorus Pesticide Exposure}

\section{Organophosphorus Pesticide Use in the United States}

Organophosphorus pesticides (OPs) refer to a group of pesticides that contain an organic phosphorus(V)-containing compound, which in most cases is $\mathrm{O}, \mathrm{O}$-dialkyl substituted, where the alkyl groups are either dimethyl or diethyl (Mileson et al. 1998). Forty OPs are currently registered for use in the United States. In 2001, the EPA banned OPs for most residential uses, although stocks were allowed to run out and container-bait traps were allowed to remain on the market. OPs also remain in use for agricultural purposes and nonresidential uses such as for golf courses, greenhouses, and for mosquito and fire ant control. The EPA recently proposed revoking food tolerances for chlorpyrifos, one commonly used OP insecticide, and will issue a final ruling in December 2016 (EPA, 2016). In 2000, 88 million pounds of OPs were applied in the United States, and by 2007 , this number had dropped to 33 million pounds (EPA 2011). Biomarker levels of OPs also dropped after 2001, although over $25 \%$ of the population still had detectable levels of OP metabolites in $2003-2004$, as did over $50 \%$ in selected subgroups (mainly Mexican-Americans and non-Hispanic Blacks) (CDC 2009).

\section{Environmental Fate, Breakdown, and Metabolism of Ops}

Use of OPs grew after environmentally persistent organochlorine pesticides were banned in the 1970's, in part because OPs are less persistent in the environment and break down fairly rapidly (within weeks to months) in the presence of sun, water, wind, and microbes. However, when used indoors, OPs may remain stable for months to years (Fenske et al. 2000), with house dust potentially acting as a long-term source of exposure. 
Breakdown and metabolism of OPs is a complex process. Several OPs do not exhibit primary toxicity in the parent pesticide form, but rather confer toxicity after undergoing enzymatic conversion to the oxon form; an oxon is a chemical in which the phosphorus-sulfur bond has been replaced by a phosphorous-oxygen bond. This oxon form is toxic and biologically active due to the electrophilicity conferred on the phosphorous ion. The oxon is then hydrolyzed, and in most cases forms a general dialkyl phosphate (DAP) metabolite (usually either dimethyl or diethyl phosphorous derivatives, including diethylphosphate, diethylthiophosphates, diethyldithiophosphates, dimethylphosphate, dimethylthiophosphate, and dimethyldithiophosphate), along with a specific metabolite moiety (such as t-CPY for chlorpyrifos and methyl chlorpyrifos, or 4-nitrophenol for methyl parathion and parathion). Seventy five percent of OPs break down into 1 to 3 of the general metabolites (Wessels et al. 2003). Figure 1 shows an example of these processes in the metabolism and breakdown of chlorpyrifos (reprinted from (Wessels et al. 2003)). However, any given pesticide-specific metabolite moiety in the urine tends to have a low frequency of detection in the US population relative to the common metabolites (Barr et al. 2004), which derive from several different OPs (see Table 1).

As a result, a common approach to the estimation of OP exposure is through summary urinary biomarker measures of the non-specific diethylphosphate ( $\Sigma \mathrm{DEPs})$ and dimethylphosphate ( $\Sigma \mathrm{DMPs}$ ) metabolites, despite the limitations of these biomarkers as internal dosimeters (Wessels et al. 2003)). In brief, these limitations include that 1) a number of different OP pesticides can be metabolized into the same DEP or DMP metabolites, preventing investigators from making any definitive link to the parent compound of exposure, 2) the different parent compounds have varying levels of toxicities, so summary DAPs may not reflect similar toxicities across populations, and 3) metabolism of the DAPs can occur either in the human body or in the environment. Exposure to a preformed DAP metabolite is non-toxic, while exposure to the parent oxons appears to confer toxicity (Mileson et al. 1998; Wessels et al. 
2003; Zhang et al. 2008). Nonetheless, use of $\Sigma$ DEP and $\Sigma$ DMP metabolites remain the most common method of exposure ascertainment in the general population, since the pesticidespecific moieties have detection frequencies that are too low to warrant widespread measurement.

Individual susceptibility to toxic effects of OPs may also vary by genotype. The conversion of the pesticide to the oxon is in part governed by cytochrome p450 metabolism, and the conversion of the oxon to the metabolites is in part governed by paraoxonase 1 (PON1) (Costa et al. 1999). A number of polymorphisms have been described in PON1 that affect either gene expression or substrate-specific catalytic efficiency. These include the coding polymorphisms Q192R and L55M, as well as the promoter polymorphism -108C>T. Roughly, Q192R determines catalytic efficiency, while L55M and -108C>T are associated with levels of the enzyme, although the latter two are in strong linkage disequilibrium and enzyme levels may only be determined by one of the two SNPs. Those with an M allele in the L55M SNP have lower PON1 levels on average (Brophy et al. 2001).The Q192R SNP is associated with faster catalytic efficiency in a substrate-specific manner. Some pesticides are more efficiently detoxified by the $Q$ allele (diazoxon, sarin, soman), while others are more efficiently detoxified by the R allele (paraoxon) (Costa et al. 1999). Further, OPs that break down into dimethyl moieties may have limited potential for detoxification by PON1 (Geldmacher-von Mallinckrodt \& Diepgen, 1988; Li et al., 2000).There is also significant overlap of enzymatic levels and activities across genotypes, and thus some researchers recommend assessing the overall "PON1 status" of an individual, and considering genotype and phenotype of PON1. Unfortunately, since PON1 levels are inducible, in some cases it is unclear whether the levels or activity reflect responsiveness to OP exposure (and thus also higher overall OP exposure) or simply baseline levels (thus reflecting higher detoxifying potential and lower OP exposure). Further complicating matters, PON1 is also important in protecting low density lipoproteins (LDL) from being oxidized, and thus may impart some protection to oxidative stress (Bhattacharyya et al. 2008; Durrington 
et al. 2001; Li et al. 2003), although the protective alleles often vary by cohort and population (Li et al. 2003).

\section{Source of Exposure}

In the general population, levels of DAPs are likely to predominantly arise from dietary exposure (Lu et al. 2006). Both parent pesticide residues and the dialkylphosphate metabolites are found on fruits and vegetables, with one study suggesting that a slight majority of the molar ratio of OP residues were preformed DAPs rather than the parent oxon or pesticide, from strawberries collected in 2003 (Zhang et al. 2008). Additionally, in a study of OP exposure using the specific metabolites for chlorpyrifos and malathion, median metabolite levels were reduced to non-detectable levels after changing children's fruit and vegetable consumption to completely organic sources for two weeks (Lu et al. 2006). Thus, metabolites measured from dietary exposure likely reflect a combination of exposure to the preformed, non-toxic DAP metabolites, and to the parent pesticide. However, in populations exposed to OPs either through agricultural or residential use (particularly in some highly-exposed residential populations prior to 2001), DAP levels may mainly reflect exposure to the parent pesticide.

\section{Associations between Prenatal Exposure to Organophosphorus Pesticides and Neurodevelopmental Outcomes}

\section{Overview}

OPs may work as developmental neurotoxins through a few different mechanisms (reviewed in (Eskenazi et al. 1999). OPs are primarily cited as acetylcholinesterase inhibitors, which results in a buildup of acetylcholine at the synaptic cleft. In acute OP poisoning, this can also cause overstimulation of nicotinic receptors. Symptoms include muscle weakness, fatigue, muscle cramps, paralysis, anxiety, headaches, and possibly coma or death. OPs also appear to be chronically neurotoxic at much lower doses, through the inhibition of acetylcholinesterase and the generation of oxidative radicals (Qiao et al. 2005), alteration of nuclear transcription 
factors (Crumpton et al. 2000), and/or alterations in serotonergic and dopaminergic synaptic activity (Aldridge et al. 2005a; Aldridge et al. 2005b).

Low level prenatal exposure to OPs has been associated with a wide range of neurological outcomes in both animal and human studies. These include a cross-sectional study that reports associations with ADHD (Bouchard et al. 2010), and prospective biomarker-based studies that report associations with decrements in attention (Barr et al. 2010; Marks et al. 2010; Rauh et al. 2006), IQ (Bouchard et al. 2011; Engel et al. 2011; Rauh et al. 2011), social responsiveness (Furlong et al. 2014), and Pervasive Developmental Disorder (Eskenazi et al. 2007), although Eskenazi et al. also reported no association with measures of attention at 24 months of age. Other studies that relied on occupational histories or self-reported residential exposure have associated OP exposure with decrements in attention, short-term memory, and design copying on the Stanford Binet IQ test (Grandjean et al. 2006; Ruckart et al. 2004). In animals, prenatal OP exposure has been associated with deficits in neurocognitive functioning (Levin et al. 2002; Timofeeva et al. 2008), impulsivity and attention problems (Cardona et al. 2011; Chanda and Pope 1996; Middlemore-Risher et al. 2010), animal models of depression (Aldridge et al. 2005a), and other behaviors (Gupta et al. 1985). Further, many of these associations have been shown to be sex-specific in animals (Levin et al. 2002; Levin et al. 2010; Slotkin et al. 2008b), and race- and sex-specific in humans (Engel et al. 2011; Furlong et al. 2014). Many of these behaviors (inattention, impulsivity, social problems) are characteristic of ADHD, but no prospective epidemiological studies have evaluated this association with a clinically validated case group, or taken advantage of clustered outcome data to examine associations with ADHD-like phenotypes of behavior, cognition, and executive functioning. In fact, no birth cohorts have previously reported on the associations between OPs and executive functioning, which is a hallmark of ADHD. Thus, prenatal exposure to OPs appears to have a wide-ranging influence on several aspects of neurodevelopment, but may actually be associated with ADHD (discussed in further detail in Chapter 2: Spotlight on ADHD). 
OPs and Neurodevelopment: Birth Cohorts

A few children's environmental health centers were formed in the late 1990's and early 2000 's to address questions of associations between prenatal exposure to neurotoxicants and reproductive outcomes and/or childhood development. These included the CHAMACOS cohort in Berkeley, with participants composed predominantly of Hispanic agricultural workers; the Mount Sinai cohort in New York City, a multiethnic cohort with participants recruited both from the Bronx and the Upper East Side of Manhattan; the Columbia cohort, a cohort of Dominican and African American women in New York City; and the Cincinnati Health Outcomes and Measures of the Environment Study (HOME) cohort, with participants recruited from the Cincinnati metropolitan area. Mount Sinai enrolled participants from 1998 to 2001, Berkeley's CHAMACOS enrolled from 1999-2000, Columbia enrolled from 1999-2006, and the Cincinnati cohort enrolled from 2003-2006. These cohorts thus vary by geography, socioeconomic status, and enrollment time period before/after the OP ban.

Reported associations between prenatal OP exposure and child neurodevelopmental outcomes have not been entirely consistent across cohorts (Table 2), which in part may be due to the fact that sources of exposure, prevalence of different parent compounds, and the ratio of of preformed DAP to parent compound exposure may vary across study cohorts. As discussed in section 1.1, cohorts typically use $\Sigma$ DEPs and $\Sigma D M P s$ as a summary measure of OP exposure (with the exception of the Columbia cohort, which has mostly relied on a blood biomarker of chlorpyrifos), but since various parent pesticides may break down into the same metabolites (Table 1), and not all of these parent pesticides have similar toxicities, the toxicity of $\Sigma$ DEPs and ¿DMPs may not be highly comparable across populations with different exposure sources. Participants in the Mount Sinai cohort were primarily exposed to pesticides used for urban pest control measures or through pesticide residues from their diet, while in CHAMACOS, participants were primarily exposed to pesticides in an agricultural setting. CHAMACOS participants had much higher levels of EDMPs than any other cohort. In the Cincinnati HOME 
study, the cohort is primarily semi-urban or suburban, and participants were generally exposed to pesticide residues in their diet, since the cohort began enrollment two years after the ban on most residential uses of chlorpyrifos and diazinon. HOME is the only cohort to have reported a positive association between OP pesticide metabolites and neurodevelopment, which is likely confounded by consumption of fruits and vegetables, the primary vector for OP metabolites in this population. Additionally, fruit and vegetable consumption may result in a high consumption of preformed metabolites, which are non-toxic and reduce the accuracy of the $\Sigma D A P$ metabolites as a measure of exposure to the toxic OP oxon. Findings from this cohort on associations between prenatal OP exposure and later neurodevelopmental outcomes have not yet been published at the time of the present study, thus, it is unknown whether these positive associations are maintained throughout childhood. Differences in source, type, and dose may explain why some studies report different associations with different metabolites (see Table 2 for summary of associations). Indeed, a recent pooled study that incorporated DAPs across the four cohorts reported substantial heterogeneity by cohort, with DMPs from CHAMACOS resulting in the strongest adverse associations with the Bayley's MDI at age 2 (S. M. Engel et al., 2015).

\section{PON1 and Other Modifying Factors}

PON1

Associations of the PON1 polymorphisms (PON1 Q192R, L55M and -108C>T) and PON1 enzyme levels have been assessed as potential modifiers (and as main effects) in the associations between prenatal exposure to OPs and childhood neurodevelopment. However, results have not been consistent with regards to the important polymorphism or the oxons that appear to be modified. For instance, Engel et al (2011) report that Q192R modifies the association between LDEPs and EDMPs and the BSID-II 12-month Bayley MDI in the Mount Sinai Cohort with stronger adverse associations among those with the QR/RR genotype. 
However, this effect is absent in the 24-month MDI and in the 6-to-7-year WISC IQs. In CHAMACOS, Eskenazi et al (2010) reported that the -108T allele itself resulted in lower MDI scores, although there was no interaction between genes and OPs on Bayley MDIs or PDIs among 2-year-olds. They also measured maternal arylesterase and paraoxonase activity levels, and reported that children of mothers with higher activity levels were slightly less likely to display PDD (pervasive developmental disorders). Regardless of inconsistent evidence, PON1 genotype and phenotype may play important roles in modifying the associations between OPs and neurodevelopment in the Mount Sinai cohort.

OPs not only impart toxicity through the mechanisms discussed earlier, but they also generate oxidative radicals (Qiao et al. 2005). Thus, while much of the research on PON1 as a modifier of organophosphorus pesticides has been driven by its potential to detoxify organophosphorus pesticides, PON1 may also be important in reducing oxidative stress responses to these pesticides.

\section{Race and Sex}

In animal models, OPs and other prenatal exposures appear to have sex specific effects (Aldridge et al. 2004; Levin et al. 2010; Slotkin et al. 2008a), and this has been partially borne out in the epidemiological literature on OPs (Furlong et al. 2014). Additionally, a small-scale fMRI study of children who were highly exposed to chlorpyrifos reported suggestive neurological differences by sex (Rauh et al. 2012). In general, the developing male brain may be more susceptible to environmental insults (Barrett 2009), although behavioral issues may be underdiagnosed in females (Begeer et al. 2013; Bruchmüller et al. 2012), possibly leading to substantial outcome misclassification which may obscure associations.

Race may be an important modifier due to differences in culture, genotype frequency, or insofar as it reflects imperfectly measured socioeconomic vulnerabilities. The strength of these differences vary by cohort; in CHAMACOS, almost all mothers were Hispanic; in Columbia, 
cohort members were restricted to blacks and Hispanics (predominantly Dominicans); in Cincinnati, mothers were predominantly upper-middle-class whites; while in Mount Sinai, participants were a mix of both whites from Manhattan and blacks and Hispanics from the Bronx and East Harlem. Notably, Mount Sinai is the only cohort to have reported any modifications by race, probably because this is the only cohort which has sufficient racial/ethnic variability, and also because the racial differences reflect drastic differences in socioeconomic status. In Mount Sinai, studies generally report stronger adverse associations between OPs and neurodevelopment among blacks, and little or no effect of OPs among whites. Cincinnati is the only cohort to have reported positive associations between OPs and any childhood outcome. These differences by race may be due to differences in exposure source, or access to resources. For instance, higher SES mothers may be more likely than lower SES mothers to access early intervention services for their children, which might obscure possible effects of pesticide exposure in these children. Race in this cohort will thus be assessed as a modifier.

\section{Other Xenobiotics and Neurodevelopmental Outcomes}

Other chemicals with different mechanisms of action also have been associated with various aspects of neurodevelopment. Phthalates have been consistently associated with alterations in behavior, executive functioning, and possibly sex development in prospective cohort studies (Engel et al. 2010; Kobrosly et al. 2014; Miodovnik et al. 2011; Swan et al. 2010; Whyatt et al. 2012; Yolton et al. 2011). Associations with IQ have been reported in crosssectional studies (Cho et al. 2010), but results from the prospective cohort studies that report prenatal phthalate associations with IQ in children over 1 year are mixed (Factor-Litvak et al. 2014; Polanska et al. 2014; Téllez-Rojo et al. 2013; Werder 2012). Phthalates may act as endocrine and thyroid disruptors (Colborn 2004), and some studies do report sex-specific effects (Cho et al. 2010; Engel et al. 2009; Kim et al. 2009; Téllez-Rojo et al. 2013). Phthalates 
may be associated with outcomes that are also associated with OP exposures, although potentially through different biological mechanisms.

Phenols (BPA in particular) have been associated with measures of behavior, typically in a sex-selective manner (Braun et al. 2009; Evans et al. 2014; Harley et al. 2013; Perera et al. 2012). Spot urine of BPA measures, however, may be an unreliable measurement of BPA throughout pregnancy, since intra-class correlation coefficients are typically $<0.20$ (Braun et al. 2010; Mahalingaiah et al. 2008).

The literature on prenatal pyrethroid insecticides and neurodevelopment is small, perhaps due to the relatively recent rise in pyrethroid use. Nevertheless, two recent studies have reported associations between prenatal exposure to pyrethroids and adverse neurodevelopment (Qi et al., 2011; Watkins et al., 2016), while another reports associations between exposure during childhood and adverse verbal and working memory scores (Viel et al., 2015). Another study found no association between prenatal permethrin exposure and neurodevelopment, but did report an association between piperonyl butoxide, a permethrin synergist, and adverse scores on the BSID-II (Bayley II) Mental Development Index in 3-yearolds (Horton et al. 2011). Finally, another study examined associations between a permethrin metabolite, PBA, and birth outcomes and head circumference, and reported no association (Berkowitz et al. 2004). In animal models, prenatal exposure to pyrethroids have been associated with altered cholinergic functioning and decreases in learning and memory performance (Sinha et al. 2006), and studies commonly report changes in motor activity and acetylcholinergic receptor density (reviewed in (Shafer et al. 2005)).

Phthalates, phenols, and pyrethroid pesticides are all relatively common environmental exposures with a wide range of suspected impacts on neurodevelopment. These exposures may confound the OP - neurodevelopment associations through correlated sources of exposure. 


\section{Confounding and the Mixture Problem}

\section{Toxicology}

Few epidemiologic studies have taken mixtures into account in the modeling process. As described above, prenatal exposure to several ubiquitous chemicals have been implicated in neurodevelopmental outcomes during childhood. These chemicals may impact neurodevelopment either by disrupting the endocrine system (Colborn 2004), through interference with neurotransmitters (Kwong 2002; Picciotto et al. 1998; Slotkin and Seidler 2008), or by interfering with DNA repair and inducing general neurotoxicity (Jamal et al. 2002). Since exposure is ubiquitous (Castorina et al. 2005; Neta et al. 2010), people are generally exposed to these and other chemicals as a mixture. However, previous studies have modeled associations as if chemical exposures occur independently. This is despite the fact that several of these chemicals have similar mechanisms of action (phthalates and some phenols are endocrine disrupting chemicals), while others have correlated reasons for use (pesticides). Additionally, exposures may be correlated due to demographic features. For example, chemical exposure overall is higher in individuals of lower socioeconomic status (Evans and Kantrowitz 2002), although this might not be true for all chemical classes. In a select few epidemiologic studies, researchers have found that accounting for mixtures produces different results from analyzing them individually (Rull et al. 2006; Wickerham et al. 2012).

In sum, accounting for mixtures may yield different results than considering compounds as if they were independent.

\section{Statistical Approaches}

In the face of substantial collinearity between predictors, standard estimates may have extremely wide variances. Additionally, power to detect an effect will be constrained as the number of covariates increases, and the model may become unwieldy and less informative as more covariates are added. Some statisticians have recommended using penalized maximum 
likelihood, which maximizes the log-likelihood but also implements a penalty favoring coefficients close to zero, in order to address these problems of high dimensionality and collinearity (Genkin et al. 2007; Tibshirani 1996).

Lasso and ridge regression are two methods that rely on penalized maximum likelihood. Lasso penalizes the sum of the absolute values of the regression coefficients, while ridge regression penalizes the sum of the squared coefficients (Genkin et al. 2007; Tibshirani 1996). Both methods stabilize the estimation and result in lower mean squared error than standard maximum likelihood estimation (MLE) by shrinking coefficient estimates toward zero. These methods work well compared to standard MLE when sample size is relatively small and the number of parameters to estimate is relatively large.

In Bayesian models, a prior distribution is assigned and estimates are shrunk towards the prior to an extent determined by the likelihood of the observed data. Estimates from the lasso produce estimates that are equivalent to the posterior estimates in a Bayesian regression that implements a double-exponential prior distribution on the coefficients (Park and Casella 2008; Tibshirani 1996). Use of independent normal priors on coefficients in a Bayesian model results in posterior estimates that are equivalent to those from a ridge regression (Griffin and Brown 2010). Ridge and lasso, however, do not account for uncertainty in the "priors" or penalties, and may result in over-shrinkage of important exposures. In Bayesian modeling, we are free to adopt alternate priors which may avoid this problem and account for uncertainty in prior assignment (Dunson et al. 2008; Herring 2010).

\section{Clustering Outcomes Data}

Prospective epidemiological cohort studies that examine the associations between environmental contaminants and childhood behavior/neurodevelopment have collected a range of neurodevelopmental instruments at various ages, motivated by a desire to obtain a broad view of that child's neurodevelopmental functioning. To date, most studies have assessed 
associations within one instrument at a time, possibly presenting the results of multiple instruments in a single manuscript, but rarely considering results across instruments with phenotypic groupings. For instance, for the Wechsler Intelligence Scale for Children- IV (WISCIV), researchers may report associations with scores on individual scales such as memory, processing speed, verbal comprehension, perceptual reasoning, and also on Full Scale IQ scores, which reflects performance on several scales. Or, if studying behavior and considering the Behavioral Assessment System for Children (BASC), researchers may report individual associations with aggression, hyperactivity, anxiety, depression, somatization, and attention. If a researcher finds associations between OPs and both behavioral measures and IQ subscales, researchers may interpret that OPs have separate impacts on both behavior and cognition. This interpretation might be faulty for several reasons reviewed below.

\section{Advantages of Clustering Outcomes Data: Clinically Relevant Outcomes}

Phenotyping of individuals using data from multiple instruments is not a unique approach. Indeed, qualitatively, this is the approach clinicians use when evaluating children for a neurodevelopmental disability. Since neurological functioning evolved as an interdependent system, functions that govern behavior are highly reliant on each other and may compensate for each other in the event of weaknesses or deficits in specific areas. For instance, "higher-level" inhibitory control relies on more "basic" processing speed capability (Ridderinkhof and van der Molen 1997). Accurate working-memory for conjunctions (or relations), which is typically considered a function of the prefrontal cortex, relies on proper functioning of the hippocampus (Olson et al. 2006). And, higher-level executive functions (EFs) are dependent on less complex EF processes, including attention, memory, perception, and categorization (Eslinger 1996). Deficits may also be compensated for by strengths in other regions; working memory alone is only associated with poor social skills when accompanied by deficits in attention, hyperactivity, and impulsivity (Kofler et al. 2011), and problems with working memory may only be associated 
with poorer academic achievement when accompanied by symptoms of inattention (Rogers et al. 2011).

Patterns of correlations across scales may also point to different clinical phenotypes when aggression problems are accompanied by high impulsivity, researchers see a different profile of aggressive behavior (reactive-impulsive) than when aggression is accompanied by higher levels of self-control (controlled-instrumental) (Vitiello and Stoff 1997), and these differences are associated with different patterns of behaviors and criminality (Nelson and Trainor 2007). Similarly, children with ADHD who display both inattention and hyperactivity, but not hyperactivity alone, have significantly lower cognitive scores than their non-ADHD counterparts (Ramos et al. 2013). Different subtypes of ADHD may have different executive function profiles, although this is a debated topic [reviewed in (Geurts et al. 2005), and discussed in further detail in section 1.5.3]. Therefore, examining performance on subscales within the context of other instruments may provide clinically relevant profiles of neurodevelopment that are also differentially associated with later life outcomes.

Advantages of Clustering Outcomes Data: Relevance to Biological Interpretations of OP Mechanisms

Clustering outcome data may help reveal mechanisms of OP toxicity. Behavioral and cognitive deficits that are clinically and statistically correlated may reflect a common etiological insult. For instance, OPs influence serotoninergic processing (Aldridge et al. 2005b; Aldridge et al. 2004; Slotkin et al. 2008b; Slotkin and Seidler 2005). Serotonin, in turn, can influence aggression, social behaviors, depression, and ADHD (Cadoret et al. 2003; Eley et al. 2004; Zoroğlu et al. 2002), and these symptoms may cluster in response to a global disruption in upstream processing like serotonergic functioning. Animal and human studies do support that OPs may be associated with those behavioral outcomes (Bouchard et al. 2010; Eskenazi et al. 2007; Furlong et al. 2014; Middlemore-Risher et al. 2010; Ricceri et al. 2003; Ricceri et al. 2006), which are perhaps mediated by serotonergic functioning (Slotkin and Seidler 2008; 
Venerosi et al. 2010). However, aggression is also influenced by non-serotonergic mechanisms, including disruptions to the frontal and prefrontal cortex, GABA, oxytocin, dopamine, and MAO-A systems (reviewed in (Nelson and Trainor 2007)). OPs do not necessarily influence all of these systems, and patterns of behaviors that are influenced by serotonin are not associated with all of these systems either. When outcomes such as aggression, are analyzed individually outside of the context of other symptoms, all aggression is lumped, including aggression that is the result of non-OP-influenced mechanisms. By instead considering behavioral and cognitive clusters, we can characterize patterns of development (such as ADHD (Castellanos et al. 2006; Mattison and Mayes 2010; Semrud-Clikeman et al. 2010; Sinzig et al. 2008)) that theoretically represent common etiologies.

While correlated deficits may reflect a common etiological insult, behavioral deficits in one area may also interfere with appropriate development of other behaviors or cognitive functions. For instance, aggression may interfere with the development of intellectual functioning (Huesmann et al. 1987), and the development of successful social skills and emotional selfregulation are potentially reliant on some levels of inhibitory control at earlier ages (Carlson and Wang 2007). Inattention and impulsivity are correlated with lower IQs later (Ramos et al. 2013). The correlation in these deficits may be in part because proper functioning in one area relies on appropriate development of earlier behaviors. Thus treating outcomes that are clinically and statistically correlated as independent may obscure the underlying mechanism of OP toxicity.

\section{Advantages of Clustering Outcomes Data: Spotlight on ADHD}

Attention Deficit Hyperactivity Disorder (ADHD) is a complex, heterogeneous disorder characterized by inattention, impulsivity, and hyperactivity. ADHD is one of the most common childhood neurobehavioral disorders, and in 2011, approximately $11 \%$ of children between 4 and 17 had ever received a clinical diagnosis of ADHD, an increase of $43 \%$ from 2003 (Visser et al. 2014). The disorder appears to be more prevalent in males than females by a factor of 2-3 to 
1, which may reflect diagnostic bias, with notable under-ascertainment among females (Bruchmüller et al. 2012). Risk factors for ADHD include having a relative with the disorder (Biederman et al. 1992), alcohol use or smoking during pregnancy (Milberger et al. 1996) (although there is controversy on whether the smoking/ADHD relationship is confounded by genetics), preterm birth, and possibly prenatal exposure to high levels of PCBs, lead, and pesticides (Polańska et al. 2013; Sioen et al. 2013).

A gold-standard ADHD diagnosis is based on observations from a clinical exam, home, and school reports. The DSM-V criteria states that children must meet at least six of the criteria established for hyperactivity and impulsivity, or six of the criteria established for inattention (criteria included in Appendix 1). Symptoms must be present in at least two different settings, and behavioral or clinical assessments are often given to provide further evidence of behavioral abnormalities.

However, this categorical construct of ADHD has been recently challenged in the literature, with psychologists proposing that a dimensional view of ADHD is a more relevant model than the categorical view (Coghill and Sonuga-Barke 2012). Children with high levels of ADHD symptomatology may not qualify for an ADHD diagnosis, but these children still often experience similar difficulties in social adjustment and academic achievement (Loe and Feldman 2007). Further, deficits that are typically considered specific to ADHD, such as deficits in basic information processing and inhibitory-based executive functioning, are linearly associated with ADHD symptoms across categories of children with no ADHD behaviors, subclinical-ADHD, and ADHD (Salum et al. 2014). In fact, most childhood behavioral disorders exist on a spectrum in the population. Oppositional defiant disorder (ODD), autism spectrum disorder (ASD), and obsessive compulsive disorder (OCD) display varying levels of trait severity among children with the disorder, and symptoms are also present at subclinical levels in the population (Coghill and Sonuga-Barke 2012; Constantino and Todd 2003; Fergusson and 
Horwood 1995). From an etiological perspective, using continuous measures of behaviors associated with ADHD may be more appropriate for assessing degree of symptomatology. Increasing symptomatology likely reflects increasing deficits in neurological functioning, which may itself have a dose-response relationship with an environmental factor, such as OPs. Using continuous measures of trait severity will also lend increased power in statistical analyses.

Recently, psychologists have shifted away from the hypothesis that attention deficits are the primary construct underlying ADHD and have moved towards the theory that ADHD may be a disorder characterized by deficits in EF, with a primary deficit in inhibitory control (Barkley 1997). This general theory has undergone much refinement in the last 15 years. Executive functioning refers to the ability to plan and execute actions with a future goal in mind. Five domains are typically considered in EF: response inhibition, planning, cognitive flexibility/set shifting, working memory, and verbal fluency. However, while deficits in EF are certainly implicated in ADHD (Marije Boonstra et al. 2005; Woods et al. 2002), these deficits also occur in people with Oppositional Defiant Disorder(ODD) and Conduct Disorder (CD), though there is substantial comorbidity among the three disorders (Biederman et al. 1991; Maughan et al. 2004). People with ADHD display deficits in non-EF cognitive areas as well (Marije Boonstra et al. 2005; Woods et al. 2002). While the specificity of EF deficits as a diagnostic tool for ADHD has been debated because of these issues (Willcutt et al. 2005), recent research in psychology has focused on examining how patterns across different domains of executive functioning are associated with $A D H D$, rather than EF as a whole.

Although deficits in executive functioning may be the primary underlying neurological dysfunction in ADHD, EFs are still only one non-specific characteristic behavioral domain of ADHD, and may differ among different ADHD subtypes. ADHD is also associated with a number of other behaviors and cognitive characteristics, and considering all of these alongside EF may enhance specificity of clusters or factors. For instance, higher-level EF functions are dependent on less complex processes, including attention, memory, perception, and categorization 
(Eslinger 1996), and ADHD is associated with other behavioral problems, such as internalizing and externalizing behaviors, aggression, and other behaviors that are assessed by the Behavioral Assessment System for Children (BASC). Social problems have also consistently been associated with ADHD. Deficits in all of these areas are likely not independent in ADHD, however, and considering them together may present a more comprehensive picture of the clinical phenotype. This approach may also help distinguish associations between OPs and specific behavioral patterns, if OPs act on one overarching pathway that affects all of these domains.

Finally, performing dimension reduction results in a small number of discrete phenotypes that can be used as outcomes. This provides an advantage in reducing type I error rates traditional models warrant the testing of associations between an exposure and each composite scale and subscale. This can lead to a high number of tests and results may be subject to multiple testing bias. However, the use of factors as outcomes limits the number of tests with a single exposure to the number of factors - typically this is under 10 tests, compared with the potential for over 50 or even 100 tests if we used the individual instrument-specific scales. Dimension reduction not only improves statistical performance by reducing type I error, but also increases the digestibility of results by only requiring the interpretation of a few results by the reader.

\section{Innovation}

Previous epidemiologic research on prenatal exposures to xenobiotics and childhood neurodevelopment has typically used the 1:1 exposure-outcome approach to describe associations. While some researchers may control for one or two other xenobiotics, such as PCBs and/or lead, most cohorts have limited power to address issues of possible interaction and confounding by more than one or two other co-exposures with the use of traditional statistical methods. Additionally, most previous research on this topic has reported associations 
with individual neurological outcomes, although many of the outcomes are correlated and interpreting associations with single subscales may be less clinically relevant and biologically meaningful. This dissertation is innovative because it a) addresses the issue of potential uncontrolled confounding between OPs and other co-exposures using methods that can account for small sample size, b) considers neurodevelopmental outcomes in a clinically meaningful manner that addresses problems of correlated outcomes and c) creates a potential model for other cohorts to use in assessing associations with neurodevelopment.

Bayesian hierarchical models allow the incorporation of prior knowledge into regression analyses, which may result in more precise "confidence intervals" (actually termed posterior or credible intervals) with less data. These models also provide flexibility in model building when there is little prior knowledge, and allows shrinkage towards chemical class means. This shrinkage process is responsible for the increase in precision and provides some advantages when using a small cohort ( $<400$ mother-child pairs). Thus, this dissertation is innovative in that it addresses problems of small sample size and uncontrolled mixture effects of co-exposures by using novel analytical methods.

Factor analysis tackles problems associated with correlated outcomes and multiple testing. This technique groups variables that are highly correlated and is one method of dimension reduction. Aside from the Faroese birth cohort that examined associations between prenatal mercury exposure and neurodevelopment (Budtz-Jørgensen et al. 2002), such techniques have been relatively under-used in neurodevelopmental epidemiology, despite their potential for collapsing neurobehavioral scores into more meaningful clinical outcomes. Current common practice is to report associations between chemical exposures and each subscale on single instruments, although associations with single subscales have little interpretability and offer no support to hypotheses about biological mechanisms of action. Using factor analysis can theoretically reveal common factors that a) may represent an underlying, meaningful biological mechanism, b) correlate with clinically meaningful neurobehaviors across instruments, and c) 
reduce the number of statistical tests performed. This approach to modeling the outcomes may provide a model for other birth cohort studies to follow, so that epidemiological research into psychological outcomes can take advantage of methods developed by psychologists and psychometricians that are designed to deal with these particular aspects of measuring neurodevelopment. 


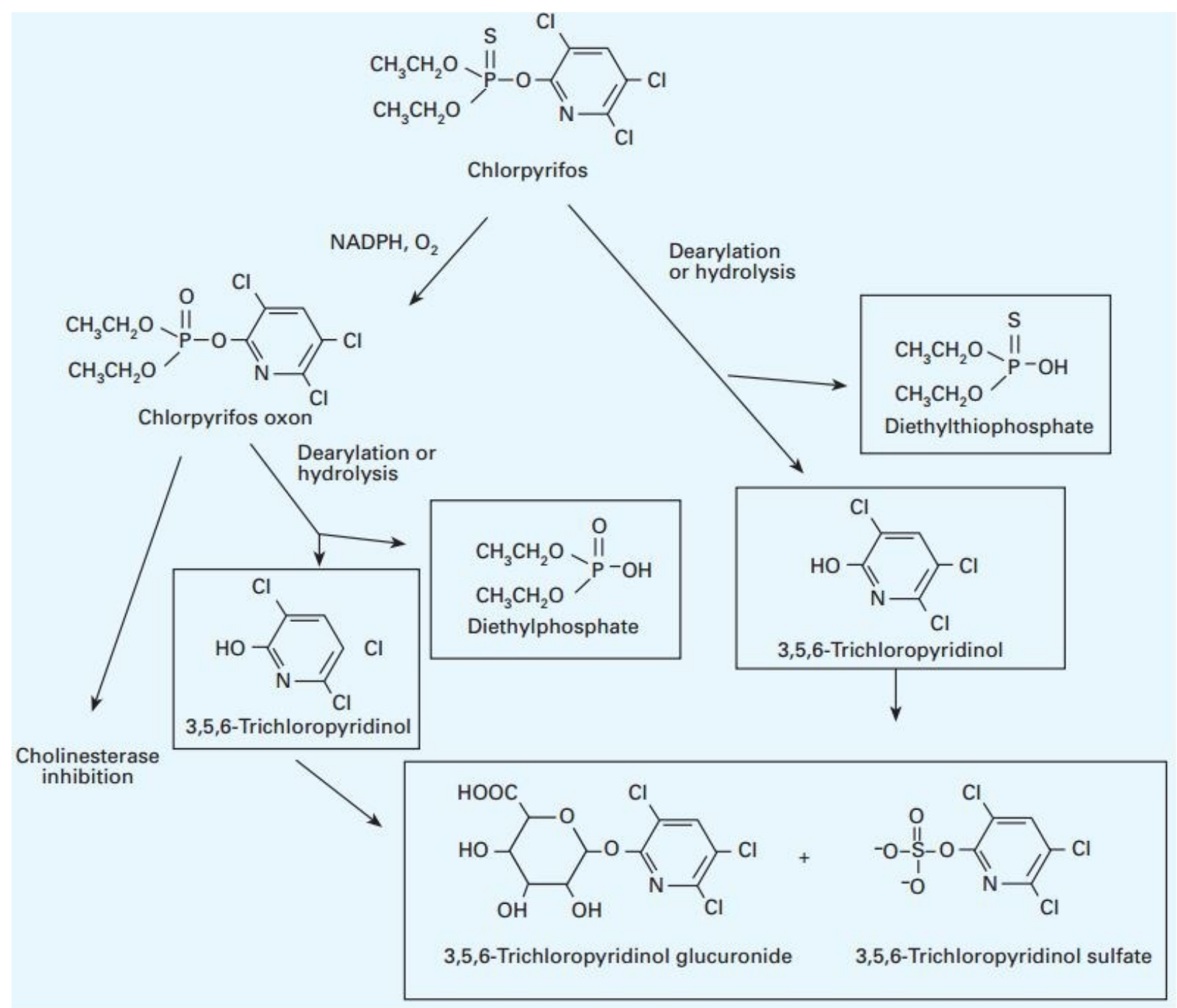

${ }^{1}$ Chemicals enclosed in boxes are excreted in the urine.

Figure 1 Chlorpyrifos Breakdown and Resulting Metabolites ${ }^{1}$ 
Table 1 Common Metabolites of Selected Organophosphorus Pesticides

\begin{tabular}{|c|c|c|c|}
\hline OP & \multicolumn{3}{|c|}{ Dialkylphosphate } \\
\hline \multicolumn{4}{|l|}{ Dimethylphosphate } \\
\hline Dichlorvos & DMP & & \\
\hline Dicrotophos & DMP & & \\
\hline Mevinphos & DMP & & \\
\hline Naled & DMP & & \\
\hline Tetrachlorvinphos & DMP & & \\
\hline Trichlorfon & DMP & & \\
\hline Acephate & DMP & DMTP & \\
\hline Chlorpyrifos methyl & DMP & DMTP & \\
\hline Fenitrothion & DMP & DMTP & \\
\hline Fenthion & DMP & DMTP & \\
\hline Methamidophos & DMP & DMTP & \\
\hline Methyl Parathion & DMP & DMTP & \\
\hline Oxydemeton methyl & DMP & DMTP & \\
\hline Primiphos methyl & DMP & DMTP & \\
\hline Temephos & DMP & DMTP & \\
\hline Azinphos methyl & DMP & DMTP & DMDTP \\
\hline Dimethoate & DMP & DMTP & DMDTP \\
\hline Malathion & DMP & DMTP & DMDTP \\
\hline Methidathion & DMP & DMTP & DMDTP \\
\hline Phosmet & DMP & DMTP & DMDTP \\
\hline \multicolumn{4}{|l|}{ Diethylphosphate } \\
\hline Chlorethoxyfos & DEP & DETP & \\
\hline
\end{tabular}




\begin{tabular}{|llll|}
\hline Chlorpyrifos & DEP & DETP & \\
Coumaphos & DEP & DETP & \\
Diazinon & DEP & DETP & \\
Ethyl parathion & DEP & DETP & \\
Sulfotepp & DEP & DETP & \\
Disulfoton & DEP & DETP & DEDTP \\
Ethion & DEP & DETP & DEDTP \\
Phorate & DEP & DETP & DEDTP \\
Phosalone & DEP & DETP & DEDTP \\
Terbufos & DEP & DETP & DEDTP \\
\hline
\end{tabular}

Reprinted from Duggan et al 2003 
Table 2 Summary of Prenatal Exposure to OPs and Associations with Neurodevelopment in Children's Environmental Health Center Cohorts

\begin{tabular}{|c|c|c|c|c|c|c|c|c|c|}
\hline \multirow[b]{2}{*}{ OP } & \multirow[b]{2}{*}{$\begin{array}{l}\text { Age } \\
\text { at } \\
\text { Test }\end{array}$} & \multicolumn{4}{|c|}{$\begin{array}{l}\text { Birth Characteristics and Measures of } \\
\text { Behavior }\end{array}$} & \multicolumn{4}{|c|}{ Measures of Cognition } \\
\hline & & $\begin{array}{l}\text { Mount } \\
\text { Sinai }\end{array}$ & $\begin{array}{l}\text { Colu } \\
\text { mbia }\end{array}$ & $\begin{array}{l}\text { CHAMAC } \\
\text { OS }\end{array}$ & $\begin{array}{l}\text { Cincinna } \\
\text { ti }\end{array}$ & $\begin{array}{l}\text { Mount } \\
\text { Sinai }\end{array}$ & $\begin{array}{l}\text { Colu } \\
\text { mbia }\end{array}$ & $\begin{array}{l}\text { CHAMAC } \\
\text { OS }\end{array}$ & $\begin{array}{l}\text { Cincinn } \\
\text { ati }\end{array}$ \\
\hline DEPs & $\begin{array}{l}\leq 1 \\
\mathrm{yr}\end{array}$ & $\begin{array}{l}\text { Abnormal } \\
\text { primitive } \\
\text { reflexes, } \\
\text { head } \\
\text { circumfer } \\
\text { ence }^{1} \text {, } \\
\text { birth }^{1} \\
\text { weight }^{1}\end{array}$ & $\begin{array}{l}\text { Birth } \\
\text { length, } \\
\text { birth } \\
\text { weight, }\end{array}$ & $\begin{array}{l}\text { Abnormal } \\
\text { primitive } \\
\text { reflexes }\end{array}$ & $\begin{array}{l}\text { Birth } \\
\text { weight }{ }^{2} \text {, } \\
\text { Improved } \\
\text { attn }\end{array}$ & & $\begin{array}{l}\text { Null } \\
\text { MDI, } \\
\text { null } \\
\text { PDI }\end{array}$ & $\begin{array}{l}\text { Null PDI, } \\
\text { MDI }\end{array}$ & \\
\hline DMPs & $\begin{array}{l}\leq 1 \\
y r\end{array}$ & $\begin{array}{l}\text { Abnormal } \\
\text { primitive } \\
\text { reflexes }^{1} \text {, } \\
\text { birth } \\
\text { length }^{1}\end{array}$ & $\begin{array}{l}\text { Gestati } \\
\text { onal } \\
\text { age }\end{array}$ & $\begin{array}{l}\text { Abnormal } \\
\text { primitive } \\
\text { reflexes, } \\
\text { gestational } \\
\text { age }\end{array}$ & $\begin{array}{l}\text { Gestational } \\
\text { age and } \\
\text { birth } \\
\text { weight }^{2}\end{array}$ & $\mathrm{MDI}^{1,2}$ & & $\begin{array}{l}\text { Null PDI, } \\
\text { MDI, }\end{array}$ & \\
\hline DEPs & $2 \mathrm{yr}$ & & & Null PDD & & $\begin{array}{l}\text { Null } \\
\text { PDI, } \\
\text { MDI }\end{array}$ & $\begin{array}{l}\text { Null } \\
\text { MDI, } \\
\text { PDI }\end{array}$ & $\begin{array}{l}\text { Null PDI, } \\
\text { MDI }\end{array}$ & \\
\hline DMPs & $2 \mathrm{yr}$ & & & PDD & & $\begin{array}{l}\text { Null } \\
\text { PDI, } \\
\text { MDI }\end{array}$ & & $\begin{array}{l}\text { Adverse } \\
\text { MDI }^{1} \text {, no } \\
\text { PDI }\end{array}$ & \\
\hline DEPs & $3 \mathrm{yr}$ & & $\begin{array}{l}\text { Attn/A } \\
\text { DHD } \\
\text { PDD }\end{array}$ & $\begin{array}{l}\text { Null } \\
\text { attn/ADHD }\end{array}$ & & & $\begin{array}{l}\text { MDI, } \\
\text { PDI }\end{array}$ & & \\
\hline DMPs & $3 \mathrm{yr}$ & & & $\begin{array}{l}\text { Null } \\
\text { attn/ADHD }\end{array}$ & & & & & \\
\hline DEPs & $\begin{array}{l}>3- \\
6 \mathrm{yr}\end{array}$ & & & $\begin{array}{l}\text { Attn \& } \\
\text { ADHD } \\
\text { indicators }\end{array}$ & & & & & \\
\hline DMPs & $\begin{array}{l}>3- \\
6 \mathrm{yr}\end{array}$ & & & $\begin{array}{l}\text { Attn \& } \\
\text { ADHD } \\
\text { indicators }\end{array}$ & & & & & \\
\hline DEPs & $\begin{array}{l}>6 \\
y r\end{array}$ & $\begin{array}{l}\text { SRS, } 7-9 \\
\text { years }^{2}\end{array}$ & & & & Null IQ & $\begin{array}{l}\text { Workin } \\
\mathrm{g} \\
\text { Memor } \\
\text { y, } \\
\text { FSIQ }\end{array}$ & $\begin{array}{l}\text { Processing } \\
\text { Speed }\end{array}$ & \\
\hline DMPs & $\begin{array}{l}>6 \\
y r\end{array}$ & $\begin{array}{l}\text { Null } \\
\text { SRS, 7-9 } \\
\text { years }\end{array}$ & & & & $\begin{array}{l}\text { Percept } \\
\text { ual } \\
\text { Reasoni } \\
\mathrm{ng}^{1}\end{array}$ & & $\begin{array}{l}\text { Working } \\
\text { Memory, } \\
\text { Verbal } \\
\text { Comprehen } \\
\text { sion, FSIQ }\end{array}$ & \\
\hline
\end{tabular}

PDI - Psychomotor Development Index of the BSID-II; MDI- Mental Development Index of the BSID-II; PDD - Pervasive Developmental Disorder as measured by the Child Behavior Checklist (CBCL); SRS Social Responsiveness Scale; FSIQ - Full Scale IQ from WISC-IV

${ }^{1}$ Modification by PON1 genotype or PON1 levels

${ }^{2}$ Modification by race and/or sex

*Many of the "null" associations reported here show a trend, but confidence intervals include the null. The Columbia cohort only reports results for chlorpyrifos, so associations with DMPs are not reported in that cohort. 


\section{CHAPTER III. METHODS}

\section{Study Design/Research Strategy Overview}

We used Bayesian hierarchical mixture models to assess the associations between organophosphorus pesticides (OPs) on neurodevelopment in a multiethnic cohort in New York City ( $n=404$ mother/infant pairs at birth, see Table 3 for demographic characteristics). Specifically, we shrunk estimates for individual xenobiotic biomarkers towards a common class mean, and also estimated these associations by assigning a more commonly used prior with a mean of 0 and variance of 1 to the individual biomarkers. The model included the OPs and controlled for phthalates, pyrethroids, and phenols. Other important modifiers, including race, sex, and PON1 genotype, were assessed for interaction with OPs in individual models. Covariates of interest are described below and were included in a DAG to determine inclusion in final models. Outcomes are continuous factor scores derived from scales of instruments collected at 6 and 7-9 years of age (instruments described in further detail in Chapter 3 Section: Outcomes, and factor analysis described in further detail in Chapter 3 Section: Factor Analysis).

\section{Study Population}

The Mount Sinai Children's Environmental Health Study is a prospective multiethnic cohort of primiparous women with singleton pregnancies who delivered at the Mount Sinai Hospital between May 1998 and July 2001 (Berkowitz et al. 2003; Berkowitz et al. 2004). Women were recruited at either the Mount Sinai Diagnostic and Treatment Center, which serves a predominantly minority East Harlem population, or at one of two private practices on the Upper East Side of Manhattan. 404 mother-infant pairs were successfully recruited during pregnancy and met inclusion criteria (i.e., free of medical complications, gestation $\geq 32$ weeks of pregnancy, infant free of genetic abnormalities, continued residence in New York City). At 
enrollment, mothers were predominantly young $(67.9 \%<25$ years $)$, non-white $(68.7 \%)$, unmarried $(71.0 \%)$, with high school educations or less (71.8\%). An additional group of women did not have prenatal data available but did have neurodevelopmental assessments performed for their children $(n=48)$. These women were not included in any analyses that required prenatal data but were included in the factor analysis.

Although the population is highly mobile due to their socioeconomic status and the cohort experienced a relatively high degree of drop out over follow up (188 out of 404 pairs were ultimately re-contacted in follow-up at either the 6 or 7-9 year visit), the missingness of the data has not substantially altered findings and women at follow-up were only slightly different than the cohort at enrollment. Women who returned for follow-up were more likely to be single at enrollment (Table 3).

\section{Exposure, Outcome, and Covariate Assessment}

Exposures, outcomes, and covariates were measured with a variety of instruments, including biomarkers, parent/caregiver surveys, and validated child assessments.

\section{Chemical Exposures}

Exposure to chemicals was assessed with biomarkers in urine or blood. Maternal blood and urine was collected at a mean gestational age of 31.2 (sd 3.7) weeks. Maternal urine samples were analyzed by the Centers for Disease Control and Prevention (Atlanta, Georgia) using gold standard techniques for measuring metabolites of organophosphorus pesticides (Engel et al. 2007), phthalates and phenols (Wolff et al. 2008), and pyrethroids (Barr et al., 2010). In the actual data analysis, values below the LOD were handled with a truncated normal distribution, and concentrations of log values above the LOD were modeled with a normal distribution. 


\section{Outcomes}

Participants were followed up when children were 4.5-5 years, 6-6.5 years, and 7-9 years of age. At follow-up, children were assessed for IQ, behavior, and executive function using validated and age-appropriate scales described below and in further detail in Table 4.

Wechsler Preschool and Primary Scale of Intelligence, 3rd edition (WPPSI-III), and Wechsler Intelligence Scale for Children, 4th edition (WISC-IV) $(\mathrm{n}=210$ children had scores from either the WPPSI or the WISC at 6-9 years; correlations between the WISC-IV and the WPPSIIII historically are 0.89) (Engel et al. 2011). Administered to children under the age of 7, the WPPSI-III provides measures of Verbal, Performance, Processing Speed, and Full-Scale IQ. The WISC-IV provides measures of Composite Verbal, Perceptual Reasoning, Working Memory, Processing Speed, and Full-Scale IQ. These composite scales are also composed of subscales (see Table 4 for full lists).

Behavior Rating Inventory of Executive Functioning (BRIEF) ( $\mathrm{n}=210$ with a measurement at any time point). The BRIEF is an 86 -item parent report questionnaire designed to assess executive cognitive function in children 5-18 years of age. Executive functions are used to achieve goals that require planning and holding in memory a multi-step sequence of thoughts or actions, to monitor and control attention and emotion, to inhibit inappropriate behaviors, and to formulate mental models based on life experiences. The BRIEF is composed of 8 clinical scales, including

1) Inhibit- ability to control impulses,

2) Shift- ability to transition between situations,

3) Emotional control- ability to modulate emotional responses,

4) Initiate- the ability to begin a task,

5) Working memory - the ability to retain information for task completion,

6) Plan/organize - the ability to anticipate future events, set goals, and develop a systematic plan of action, 
7) Organization of materials - the ability to keep workspace orderly, and

8) Monitor - the ability to assess personal performance and to register the effect of one's own behavior on others.

These eight scales generate two broad indices: the Behavioral Regulation Index (BRI) and the Metacognition Index (MI). An overall score, the Global Executive Composite (GEC), is obtained from the raw scores for the $\mathrm{MI}$ and $\mathrm{BRI}$.

Behavioral Assessment Scale for Children (BASC) ( $\mathrm{n}=210$ with a measurement at any point). The BASC is designed to evaluate problematic behaviors in children and adolescents 2.5 to 18 years of age. The BASC includes nine clinical scales to assess a child's adaptive and problem behaviors in home and community settings. Parents respond to 130 items on a 4 point scale that ranges from never to almost always. Externalizing problems is a composite scale derived from the hyperactivity (including both hyperactivity and impulsivity items), aggression, and conduct problems scale items. Internalizing problems is a composite of the anxiety, depression, and somatization scale items. Three additional scales are attention problems, atypicality, and withdrawal. The adaptive behavior skills composite combines information from the adaptability, social skills, and leadership scale items. The Behavioral Symptoms Index (BSI) is the apical summary score that assesses the overall level of behavioral functioning.

Taken together, these instruments provide details on several aspects of neurodevelopment, including IQ, behavior, and executive functioning. Table 4 describes the instruments used and when they were administered. All outcomes were analyzed as continuous variables.

\section{Covariates}

Covariates of interest for the OP-neurodevelopment relationships included maternal education, maternal race/ethnicity, maternal marital status, alcohol use during pregnancy, drug use during pregnancy, maternal age, child sex, maternal IQ, HOME environment scores, and 
maternal smoking during pregnancy. All prenatal covariates were obtained through a questionnaire administered at the time of biomarker collection (mean gestational age 31.2 weeks). Child sex and other features of the delivery were determined through the perinatal database at the hospitals where they were delivered.

\section{Data Analysis}

The proposed aims seek to 1) comprehensively account for correlations among the outcomes and to reduce the dimensionality of these outcomes in a factor analysis, 2) to estimate associations between prenatal and early life characteristics and the neurodevelopmental factors and to 3) consider OPs as part of a mixture, and to account for possible correlations and confounding by other xenobiotics in Bayesian hierarchical models.

\section{Outcomes: Factor Analysis}

Factor analysis (FA) allows items that are highly correlated with each other to cluster. The degree to which any given variable contributes to that overall factor is known as a factor loading. Squared factor loadings represent the amount of variance explained by any given variable for that factor. In deciding what factor loading values indicate an important variable, generally analysts use a rule of thumb of loadings $>0.3$ to indicate important variables (Field, Miles, \& Field, 2012). Loadings are also related to the eigenvalues, which indicate the substantive importance of a factor. Relatively high eigenvalues indicate that a factor should be retained, while relatively low eigenvalues indicate that a factor should be discarded. A general rule of thumb that is commonly used dictates that eigenvalues above 1 should be retained, although more sophisticated methods are also available for choosing number of factors. A scree plot plots each eigenvalue against the factor with which it is associated, and generally is characterized by a steep decline followed by a leveling off. In parallel analysis, a random dataset with the same number of observations and variables is drawn, eigenvalues are estimated, and the scree plots are compared. When the eigenvalues from the observed dataset 
dip below those from the randomly drawn data, this indicates that the later factors are mostly noise. We considered both the eigenvalue cutoff method and the factor solution that is one above and below the number indicated by parallel analysis.

Factor rotation methods impose either a correlated or uncorrelated structure on the factors. In etiological analyses, we primarily adopted an orthogonal varimax rotation, in order to assess associations with factors that are linearly independent of other neurodevelopmental domains. However, in Aim 1 we compared models using orthogonal rotation, oblique rotation, and instrument-specific composite scores, to evaluate the utility of adopting the varimax rotation.

Factor scores are assigned to each participant to measure how highly they rate on any given factor. For instance, if a participant scores highly on a factor that measures problems with attention, then that participant likely displays more problems with attention than the rest of the cohort. Similarly, if a participant has a low score on that factor, then she has fewer attention problems than the rest of the participants in that cohort. These scores can be extracted and used as outcomes in the Bayesian methods described in Chapter 3. To determine factor scores, we used the standardized factor score regression coefficients.

In sum, we used analysis with orthogonal varimax rotation, eigenvalue cutoffs, and parallel analysis to decide on the correct number of factors, factor loadings above 0.30 to determine variable retention, and standardized factor score regression coefficients to determine factor scores. Scores from the neurodevelopmental instruments were scaled using z-scores for comparability in the factor analysis. Due to sample size restrictions, we used the averages of the subscale scores across visits, and included behavioral subscale scores and IQ composite scores into a FA. Since the WPPSI and the WISC do not have exactly overlapping composite scores and subtest scores, we used the composite scores that overlap (Full Scale IQ, Verbal IQ, and Processing Speed), and the overlapping subtest scores for the finer-level dimension reduction (See Table 4 for included scales). 


\section{Association Models}

For Aim 1, we used a series of multivariable linear regressions to estimate associations between prenatal exposure to smoking and other early life characteristics and neurodevelopmental factors. Aim 2, however, involves correlated exposures with a potentially high number of variables relative to sample size. Linear regressions of models with such characteristics can produce effect estimates that are notoriously unstable and inflated. Therefore, in Aim 2 we used a hierarchical Bayesian mixture model (BMM) to account for both correlated variables and to stabilize models with high numbers of parameters relative to the sample size (de Vocht et al. 2012; Herring 2010; Wakefield et al. 2010). Bayesian statistics allows for the incorporation of prior knowledge into analyses, typically with the result of shrinking effect estimates towards the specified prior and creating more precise posterior $95 \%$ intervals. In this dissertation, we shrunk estimates towards a mean of 0 with a variance of 1 , and performed sensitivity analyses where estimates were shrunk towards chemical class means.

Exposure values below the LOD were modeled as truncated normal, with values above the LOD modeled using log normal distributions (Herring 2010). Missing covariate values were imputed using full information maximum likelihood methods under the missing-at-random assumption (MAR). Modeled outcomes were factor scores derived from structural equation models/factor analysis described further in Chapter 3.

\section{Comparative Analyses}

For comparison purposes, we also presented results from a traditional linear regression that considers associations between OPs and the composite scores of the instruments, along with the other instrument-specific items that comprise the factors. These models used covariates in a minimally sufficient set derived from a DAG. 
Table 3 Characteristics of Study Population at Enrollment and Follow Up, Mount Sinai Children's Environmental Health Cohort

\begin{tabular}{|c|c|c|c|}
\hline & $\begin{array}{c}\text { Enrolled at } \\
\text { Birth } \\
N=404\end{array}$ & $\begin{array}{c}\text { Followed-up } \\
\text { ages } 6-9 \\
\text { years } \\
\mathrm{N}=162^{1}\end{array}$ & $\begin{array}{c}\text { Not Followed- } \\
\text { Up ages 6-9 } \\
\text { years } \\
N=242\end{array}$ \\
\hline \multicolumn{4}{|l|}{ Maternal Age at delivery (years) } \\
\hline$<20$ & $142(35.2)$ & $50(30.9)$ & $92(38.0)$ \\
\hline $20-24$ & $132(32.7)$ & $55(34.0)$ & $77(31.8)$ \\
\hline$\geq 25$ & $130(32.2)$ & $57(35.2)$ & $73(30.2)$ \\
\hline \multicolumn{4}{|l|}{ Maternal Race/ethnicity } \\
\hline White & $86(21.3)$ & $30(18.5)$ & $56(23.1)$ \\
\hline Black/other & $118(29.2)$ & 50 (31.9) & $68(28.1)$ \\
\hline Hispanic & $200(49.5)$ & $82(50.6)$ & $118(48.8)$ \\
\hline \multicolumn{4}{|l|}{ Maternal Education during pregnancy } \\
\hline High School or Less & $288(71.8)$ & $118(73.3)$ & $170(70.8)$ \\
\hline Some College or higher & $113(28.2)$ & $43(26.7)$ & $70(29.2)$ \\
\hline \multicolumn{4}{|l|}{ Marital Status during pregnancy ${ }^{2}$} \\
\hline Married & $117(29.0)$ & $37(22.8)$ & $80(33.1)$ \\
\hline Living with Partner & $98(24.3)$ & $37(22.8)$ & $61(25.2)$ \\
\hline Single/Divorced/Widowed & $189(46.8)$ & $88(54.3)$ & $101(41.7)$ \\
\hline \multicolumn{4}{|l|}{ Alcohol use during pregnancy } \\
\hline None & $337(85.1)$ & $132(83.0)$ & $205(86.5)$ \\
\hline Any & $59(14.9)$ & $27(17.0)$ & $32(13.5)$ \\
\hline \multicolumn{4}{|l|}{ Smoking during pregnancy } \\
\hline No smoking during pregnancy & $337(83.4)$ & $134(82.7)$ & $203(83.9)$ \\
\hline Any smoking during pregnancy & $67(16.6)$ & $28(17.3)$ & $39(16.1)$ \\
\hline $\begin{array}{l}\text { Smoking only during } 1^{\text {st }} \\
\text { trimester }\end{array}$ & $46(11.4)$ & $17(10.5)$ & $29(12.0)$ \\
\hline $\begin{array}{l}\text { Any smoking during } 2^{\text {nd }} \text { or } 3^{\text {rd }} \\
\text { trimester }\end{array}$ & $21(5.2)$ & $11(6.8)$ & $10(4.1)$ \\
\hline
\end{tabular}

${ }^{1}$ Population included in the multivariate adjusted regression models of associations between early life characteristics and the factor scores.

${ }^{2}$ Marital status at baseline of mothers from the original birth cohort $(n=404)$ who returned for follow up differed from mothers who did not return $(p=0.03)$. No other characteristics were significantly different at the alpha $=0.05$ level for these two groups. 
Table 4 Instruments Included in Factor Analysis of Behavior, Executive Functioning, and IQ

\begin{tabular}{|c|c|c|}
\hline Instrument & Scales & $\begin{array}{l}\text { Age } \\
\text { Assessed, } \\
\mathrm{N} \text { children }\end{array}$ \\
\hline $\begin{array}{l}\text { Wechsler Preschool and } \\
\text { Primary Scales of } \\
\text { Intelligence (WPPSI-III) }\end{array}$ & $\begin{array}{l}\text { Verbal IQ (subtest: Vocabulary), } \\
\text { Performance IQ (subtests: Block Design, Matrix } \\
\text { Reasoning, Picture Concepts) } \\
\text { Processing Speed Index (subtests: Symbol } \\
\text { Search, Coding) } \\
\text { Full Scale IQ }\end{array}$ & $\begin{array}{l}6 \text { years } \\
(n=162)\end{array}$ \\
\hline $\begin{array}{l}\text { Wechsler Intelligence Scale } \\
\text { for Children (WISC-IV) }\end{array}$ & $\begin{array}{l}\text { Verbal IQ (subtests: Vocabulary), } \\
\text { Perceptual Reasoning (subtests: Block Design, } \\
\text { Matrix Reasoning, Picture Concepts) } \\
\text { Processing Speed Index (subtests: Symbol } \\
\text { Search, Coding) } \\
\text { Full Scale IQ }\end{array}$ & $\begin{array}{l}7-9 \text { years } \\
(n=161)\end{array}$ \\
\hline $\begin{array}{l}\text { Behavior Rating Inventory of } \\
\text { Executive Functioning } \\
\text { (BRIEF) }\end{array}$ & $\begin{array}{l}\text { Behavioral Regulation Index (subtests: Inhibit, } \\
\text { Shift, Emotional Control) } \\
\text { Metacognition Index (Initiate, Working Memory, } \\
\text { Plan/Organize, Organization of Materials, } \\
\text { Monitor) } \\
\text { Global Executive Composite }\end{array}$ & $\begin{array}{l}\text { 4-9 years } \\
(\mathrm{N}=242)\end{array}$ \\
\hline $\begin{array}{l}\text { Behavioral Assessment } \\
\text { Scale for Children (BASC) }\end{array}$ & $\begin{array}{l}\text { Externalizing Problems (Aggression, } \\
\text { Hyperactivity, Conduct Problems) } \\
\text { Internalizing Problems (Anxiety, Depression, } \\
\text { Somatization,) } \\
\text { Adaptive Skills composite (Adaptability, } \\
\text { Leadership, Social Skills) } \\
\text { Other Problems (Atypicality, Withdrawal) } \\
\text { Behavioral Symptoms Index (Aggression, } \\
\text { Hyperactivity, Anxiety, Depression, Attention, } \\
\text { Conduct Problems, Atypicality) }\end{array}$ & $\begin{array}{l}\text { 4-9 years } \\
(\mathrm{N}=238)\end{array}$ \\
\hline
\end{tabular}

210 participants had the BASC, the BRIEF, and either the WPPSI-III or the WISC-IV

BRIEF items and descriptions:

The Behavioral Regulation Index includes these clinical scales:

- Inhibit (the ability to control impulses),

- $\quad$ Shift (the ability to switch between activities and tolerate change),

- Emotional Control (the ability to regulate emotional responses appropriately).

The Metacognition Index includes these clinical scales:

- Initiate (the ability to begin activities and generate problem-solving strategies),

- Working Memory (the ability to hold information when completing a task),

- Plan/Organize (the ability to set goals, develop steps, and anticipate events),

- Organization of Materials (the ability to put work, play, and storage spaces in order), and

- Monitor (the ability to check one's own work and performance). 


\section{CHAPTER IV. EARLY LIFE CHARACTERISTICS AND NEURODEVELOPMENTAL PHENOTYPES IN THE MOUNT SINAI CHILDREN'S ENVIRONMENTAL HEALTH CENTER}

\section{Introduction}

Neurodevelopment is a complex interdependent system, and yet epidemiological studies of neurodevelopment generally consider performance across dimensions of neurodevelopment in isolation. For example, studies may report on the relationship between a sociodemographic variable and childhood intelligence, executive functioning, or behavior, but not on all three dimensions simultaneously. Modern approaches have emphasized the need for "deep phenotyping" (Robinson, 2012). This approach uses statistical models that reflect the known complexity and interrelatedness of neurodevelopmental processes, and considers sources of variability within and across developmental or psychopathological domains (Baynam et al., 2015; Calkins et al., 2015; Robinson, 2012).

One statistical approach to deep phenotyping involves dimension reduction techniques, which take advantage of information in patterns of covariance across instruments. There are major conceptual advantages in jointly modeling domains of neurodevelopment. Accounting for interrelatedness between developmental domains may be more clinically relevant, since behavioral traits rarely present in isolation. This may be particularly true for children with a pathological diagnosis. For instance, the hallmarks of an ADHD diagnosis include problems with attention, hyperactivity, executive functioning, and impulsivity (Barkley, 1997). Patients with ADHD often also present with anxiety, conduct disorder, depression, and difficulties in forming social relationships (Jensen, Martin, \& Cantwell, 1997). Even in a general population sample, traits such as anxiety and depression are often highly comorbid (Sartorius, Ustun, Lecrubier, \& Wittchen, 1996), implying potentially common neurological underpinnings for these traits. 
Despite these co-occurring patterns, there is a trend in etiological studies to either focus on a holistic outcome such as ADHD, or to adopt a trait-driven approach and to assume characteristics that such as anxiety or depression are independent. However, in a general population sample, a more nuanced approach that accounts for correlational patterns across traits may be more neurologically and clinically relevant.

Many perinatal, social, behavioral, environmental, and demographic characteristics have been associated with individual dimensions of neurodevelopment (Bradley, Caldwell, Rock, \& Ramey, 1989; Gale et al., 2008; Indredavik, Brubakk, Romundstad, \& Vik, 2007; Piper \& Corbett, 2012; Willford, Leech, \& Day, 2006). However, few etiological studies have attempted to jointly model domains of neurodevelopment while accounting for their interrelationships. As neurological capabilities scaffold into phenotypes during childhood, examining the associations between prenatal and early life characteristics and neurodevelopmental phenotypes may provide more insights into underlying etiological pathways. Our goal was to estimate associations between perinatal, social, demographic and behavioral characteristics and neurodevelopmental phenotypes while accounting for outcome interdependencies using a phenotyping approach.

\section{Methods}

\section{Study Population}

The Mount Sinai Children's Environmental Health Study is a prospective cohort study of primiparous women with singleton pregnancies who delivered at the Mount Sinai Hospital in New York City between May 1998 and July 2001 (Berkowitz et al., 2003, 2004). Mother/infant pairs were followed from pregnancy until the child was 7-9 years of age. Women were recruited during prenatal visits at either the Mount Sinai Diagnostic and Treatment Center, which serves a predominantly East Harlem population, or at one of two private practices on the Upper East Side of Manhattan. Exclusions have been detailed elsewhere (S. M. Engel et al., 2007). After 
exclusions, there were 404 women with available birth data. Additionally, a small number of women who enrolled after birth and participated in follow-up visits are included in the neurodevelopmental factor analysis $(n=48)$. Participants were invited to return for neurodevelopmental follow-up visits with their child at ages 1, 2, 4, 6, and 7-9 years (Figure 1).

\section{Child Behavior and Executive Functioning}

We measured children's executive functioning with the Behavior Rating Inventory of Executive Functioning (BRIEF). The BRIEF is a parent-report assessment of the child's executive functioning (Gioia, Isquith, Guy, \& Kenworthy, 2000), which consists of 86 items that are rated on a 3-level scale from "never" to "almost always". Validity studies report good reliability with high test-retest reliability (mean $r_{\mathrm{s}}=0.81$ for parents across scales) and internal consistency (Cronbach's alphas range from 0.80-0.98 across scales)(Gioia et al., 2000). Individual items are summarized into eight clinical scales and their age-normed T-scores. Clinical scales are then collapsed into two indices, which also include age-normed T-scores. The Behavioral Regulation Index includes the following three clinical scales: Inhibit (the ability to control impulses), Shift (the ability to switch between activities and tolerate change), and Emotional Control (the ability to regulate emotional responses appropriately). The Metacognition Index includes five clinical scales: Initiate (the ability to begin activities and generate problemsolving strategies), Working Memory (the ability to hold information when completing a task), Plan/Organize (the ability to set goals, develop steps, and anticipate events), Organization of Materials (the ability to put work, play, and storage spaces in order), and Monitor (the ability to check one's own work and performance). The Behavioral Regulation and Metacognition indices are then combined into one overarching Global Executive Composite score. Mothers completed the BRIEF at the 4, 6, and 7-9 year follow-up visits. We used the average T-scores across visits.

We assessed children's problem and adaptive behaviors in the home and community setting with the parent report version of the Behavioral Assessment System for Children (BASC) 
(Reynolds \& Kamphaus, 1998; Sandoval \& Echandia, 1994). Internal consistency reliability of this instrument is good (Cronbach's alphas average 0.80 across scales and ages), and testretest reliabilities are also high (mean $r_{\mathrm{s}}=0.85$ for preschool, mean $r_{\mathrm{s}}=0.87$ for children ages 6-11)(Sandoval \& Echandia, 1994). Parents completed a survey consisting of over 200 items that describe the frequency of a specific behavior on a four point scale from "Never" to "Almost Always". Items are then collapsed into clinical and adaptive scales, which are normed to the general population by child age to produce T-scores. Clinical and adaptive scales are then consolidated into composites, which are also age normed. These composites included Externalizing Problems (Aggression, Hyperactivity, Conduct Problems), Internalizing Problems (Anxiety, Depression, Somatization), Adaptive Skills (Adaptability, Leadership, Social Skills), and the Behavioral Symptoms Index (Aggression, Hyperactivity, Anxiety, Depression, Attention Problems, Atypicality). Withdrawal is the only scale that is not included in a composite. The BASC was completed at the 4, 6, and 7-9 year visits, and we used the average T-scores across visits.

\section{Psychometric Intelligence Testing}

Children were administered the Wechsler Preschool and Primary Scales of IntelligenceIII (WPPSI-III) at age 6 (mean age $=6.2, \mathrm{SD}=0.2$ ), and the Wechsler Intelligence Scales-IV (WISC-IV) between the ages of $7-9$ years (mean age $=7.8, \mathrm{SD}=0.8$ ). The WPPSI-III subtests included in this analysis were Block Design, Matrix Reasoning, and Picture Concepts (for the Performance IQ), Symbol Search and Coding (for Processing Speed), and Vocabulary (for the Verbal IQ). A Full Scale IQ (FSIQ) is generated from the age-normed composites. The assessed WISC-IV subtests were Block Design, Matrix Reasoning, and Picture Concepts (for Perceptual Reasoning), Vocabulary (for Verbal Comprehension), and Coding and Symbol Search (for Processing Speed). Because the FSIQ from the WPPSI-III and WISC-IV are highly correlated $\left(r_{\mathrm{s}}=0.84\right.$ in our population), if a child returned for both visits, we preferentially used 
the WISC-IV scores for all subtests. In our analyses, we were only able to include subscales that were administered in both the WPPSI-III and the WISC-IV. Thus, from the WPPSI-III, we excluded Word Reasoning and Information. From the WISC-IV we excluded Digit-Span, LetterNumber Sequencing, the Working Memory Index, Similarities, and Comprehension (see Table 5 for full list of included scales).

\section{Covariates}

During their third trimester and at follow-up visits, mothers completed questionnaires that assessed a variety of sociodemographic, occupational, environmental, medical history and behavioral characteristics. We classified maternal characteristics as follows: maternal education at follow-up (high school or less vs some college or higher), maternal age $(<20,20-25,>25)$, maternal race/ethnicity (white/non-white), smoking during pregnancy (we examined both a binary form of this variable that included any smoking during pregnancy vs no smoking, and a three-level categorical variable that included no smoking, smoking in first trimester only, and smoking in either second or third trimesters), alcohol use during pregnancy (ever/never), and canned fish consumption during pregnancy ( $\geq 1$ time per week or $<1$ time a week).

A perinatal database at the Mount Sinai Department of Obstetrics, Gynecology, and Reproductive Science was used to abstract delivery characteristics and birth outcomes, including head circumference, birth weight, birth length, and gestational age. We categorized gestational age into preterm ( $<37$ weeks) or term (37 weeks or more) and used a continuous measure of head circumference (centimeters). Birth weight and length were dichotomized at the median ( $<51 \mathrm{~cm}$ vs $\geq 51 \mathrm{~cm}$ for birth length; $<3270$ vs $\geq 3270$ grams for birth weight).

An in-office interview version of the Home Observation for Measurement of the Environment (HOME scale) (Caldwell \& Bradley, 1984) was administered at 12 and 24 months. The HOME subscales include 1) Involvement, which measures how an adult interacts physically with the child (sample items include: parent keeps child within visual range, talks to child while 
doing work); 2) Learning Materials, which measures whether a child has appropriate play materials at home and elsewhere (sample items include: child has one or more large muscle activity toys); 3) Organization, which measures how a child's time is organized outside the house and what the child's personal space looks like (sample items include: safe play environment, regular caregivers); 4) Acceptance, which measures how the adult disciplines the child (sample items include: parent does not shout at child during the visit, parent not overly restrictive of the child's movements), 5) Responsivity, which measures the emotional and verbal sensitivity and responsivity of parent to the child (sample items include: mother caresses or kisses child at least once during visit), and 6) Variety, which measures opportunities for variety in daily stimulation (sample items include: father provides some caregiving every day, family visits or receives visits from relatives approximately once a month).

We used mean overall HOME scores and HOME subscale scores across the 1-2 year visits. The HOME overall score exhibited a wide range with sufficient variability across scores and was included as a continuous variable. However, the HOME subscale scores exhibited a limited range with most observations clustered at the higher, more positive end of the distributions. We thus categorized the HOME subscale scores into tertiles and included them as ordinal categorical variables. The HOME subscale scores met assumptions for linearity when categorized in this fashion.

Maternal intelligence was assessed during pregnancy using the Peabody Picture Vocabulary Test-III (Dunn \& Dunn, 1997) and the covariate was defined as a continuous variable. 


\section{Statistical Methods}

\section{Characteristics by Follow-Up Status}

We examined demographic characteristics of mothers by follow-up and covariate status. We used chi-square goodness-of-fit tests with an alpha cutoff of 0.05 to assess if mothers from the original birth cohort $(n=404)$ who returned for follow-up and completed the BASC, the BRIEF, the WPPSI-III/WISC-IV differed from mothers who did not return for a complete followup visit. Maternal education and marital status at baseline were used to assess differences by follow-up status, since maternal education and marital status at follow-up are unavailable for those who did not return for a follow-up visit.

Factor Analysis

We performed dimension reduction on the BASC, BRIEF, WPPSI-III, and WISC-IV by using a principal components analysis with an orthogonal varimax rotation and present loadings above the cutoff of 0.3. We included standardized versions of both the composite scores and the subscales of the instruments. The neurodevelopmental scales included in this analysis are listed in Table 1. We examined criteria for factorability, including Kaiser's measure of sampling adequacy and Bartlett's test of sphericity, both of which assess the suitability of the data for factor analysis based on correlations among the variables (Dziuban \& Shirkey, 1974). We also examined communalities to assess suitability of items, and average communality size to assess adequacy of sample size (MacCallum, Widaman, Zhang, \& Hong, 1999). To determine the number of factors, we examined factors with eigenvalues greater than one, and also considered parallel analysis to optimize the number of factors selected (Hayton, Allen, \& Scarpello, 2004). Parallel analysis computes eigenvalues from a correlation matrix that is derived from a random dataset with the same numbers of observations and variables as the original data, and compares them against the eigenvalues from the observed data. We then examined the factor solution that was one higher and one lower than that which was indicated by parallel analysis. In order to aid interpretation, we scaled factors so that positive/negative attributes go in the same 
direction across all factors in the regression analyses. Thus, positive scores on all of the factors indicate better outcomes and negative scores indicate more adverse outcomes in all regression analyses. For regression analyses, we standardized all factors to have a mean of 0 and a standard deviation of 1 . Thus, a beta coefficient of one can then be interpreted as an increase of one standard deviation of the factor. In sensitivity analyses, we assessed whether the factor structure differed for whites and non-whites and also assessed the factor structure with a promax rotation.

Association Analyses of Early Life Characteristics with Neurodevelopmental Factors We estimated associations between characteristics hypothesized to be associated with neurodevelopment, and orthogonal varimax-rotated factors, in mutually adjusted analyses. We considered covariates that have previously been hypothesized to be associated with neurodevelopment. These covariates include maternal age at enrollment, maternal education at follow-up, maternal marital status at follow-up, maternal IQ, maternal race, smoking during pregnancy, alcohol consumption during pregnancy, canned fish consumption during pregnancy, birth head circumference, preterm birth, birth weight, birth length, child sex, and HOME environment scores. To address potential issues with collinearity, we eliminated some covariates that were highly correlated (variance inflation factor $>2$ ). When covariates were strongly correlated, we retained the variable that was the strongest predictor in bivariate tests. The birth characteristics of birth length, birth weight, and preterm birth were correlated; of this set, preterm birth was retained. Maternal age, maternal marital status, and maternal IQ were dropped due to their high correlation with maternal race in this dataset, which was the strongest predictor for a number of factors. We did, however, perform sensitivity analyses including these three dropped covariates (maternal age, maternal marital status, and maternal IQ). The final covariate set for the primary analyses included maternal education, maternal race, smoking during pregnancy, alcohol consumption during pregnancy, canned fish consumption during pregnancy, birth head circumference, preterm birth, child sex, and the HOME subscale scores 
of Organization, Learning Materials, Involvement, and Variety. We multiplied imputed missing covariate data and estimated associations between early life characteristics and factor scores in multivariable linear regression models (PROC GLM with PROC MI and MIANALYZE in SAS, 10 imputations). No adjustment for multiple comparisons was performed. In sensitivity analyses, we evaluated whether associations were different by white race.

In order to evaluate whether using the phenotypes resulted in different effect estimates compared to a more traditional approach of using instrument-specific composite scores, we performed a case study of a single exposure with well-characterized associations with neurodevelopment, smoking. We estimated associations between smoking and neurodevelopment as measured by the phenotypes, and also estimated associations between smoking and neurodevelopment as measured by the highest loading composite item from each factor (i.e., the BASC's Externalizing Composite, the BRIEF's Metacognition Index, the BASC'S Internalizing Composite, the WISC-IVMPPSI-III's Perceptual Reasoning IQ score, the BASC's Adaptive Skills Index, the WISC-IV/WPPSI-III's Processing Speed IQ score, and the WISCIV/WPPSI-III's Verbal IQ score). In these analyses, we did not adjust for birth characteristics as they may be intermediate on the causal pathway between smoking and neurodevelopment. The final adjustment set was otherwise the same as in the primary analyses.

All analyses were performed in SAS V9.4.

\section{Results}

\section{Study Population Characteristics and Follow-up}

Of the 404 eligible women who enrolled during pregnancy, 162 returned for at least one visit between 6-9 years and completed the BASC, the BRIEF, and the WPPSI-III/WISC-IV. An additional 48 women who enrolled after birth had at least one complete visit between 6-9 years, although these women had no prenatal data available (Figure 2 and Table 6). 210 participants in total returned for at least one complete visit between ages 6-9 years, and these participants 
were all included in the factor analysis. The majority of the 162 participants with prenatal data were young (64.9\% under 25 at delivery) and non-white (82.5\%). Most participants reported not drinking alcohol (83.0\%), not smoking during pregnancy $(82.7 \%)$, and most had an education of high school or less at enrollment (73.3\%; Table 6). The distributions of education at enrollment, maternal age at delivery, race, alcohol consumption during pregnancy, and smoking during pregnancy were generally similar among those who did and did not return for follow-up, although mothers who were single, divorced, or widowed were more likely to return for follow-up than mothers who were married $(p=0.03)$.

\section{Principal Components Analysis}

We examined several criteria for factorability of the neurodevelopmental outcome data. Kaiser's measure of sampling adequacy was 0.71 , above the standard of 0.60 (Field et al., 2012), and Bartlett's test of sphericity was significant $(x 2(666)=13,875, p<0.01)$. Parallel analysis indicated six factors had eigenvalues greater than those generated from random data, while seven factors had eigenvalues greater than one. After examining the two solutions, we determined that the seven factor solution was almost equivalent to the six factor solution, with the seven factor solution including a separate factor for verbal intelligence. In the six factor solution, the items for verbal intelligence loaded with perceptual reasoning items. We selected the seven factor solution because perceptual reasoning and verbal intelligence capture different aspects of intelligence (W. Johnson \& Bouchard, 2005), and it had both good statistical fit based on the eigenvalues and was in line with previous literature on neurodevelopment. All neurodevelopmental scales loaded on at least one factor at $>0.30$, and all had sufficiently high communalities (all scales had communalities $>0.50$, and the average communality was 0.79 ), thus all scales were retained. Factor structures were similar for varimax and promax rotation. In order, the seven unrotated factors explained $37.92 \%, 13.71 \%, 7.86 \%, 6.33 \%, 5.10 \%, 4.25 \%$, and $3.05 \%$ of the variance in the data, for a total of $78.22 \%$. 
In order of variance explained, these seven factors are herein described as: 1) Impulsivity/Externalizing, 2) Executive Functioning, 3) Internalizing, 4) Perceptual Reasoning, 5) Adaptability, 6) Processing Speed, and 7) Verbal Intelligence (Table 7). The Impulsivity/Externalizing factor was composed primarily of the BASC's Externalizing Problems composite and associated subscales, and the BRIEF's Behavioral Regulation Index (BRI) and BRI subscales. The Executive Functioning factor was primarily characterized by the Metacognition items from the BRIEF, although items from the BASC Behavioral Symptoms Index (BSI) also loaded weakly on to this factor. Items with high loadings for the third factor, Internalizing, included the BASC's Internalizing Problems composite and the associated subscales of Anxiety, Somatization, and Depression. Withdrawal also loaded highly on this factor, although it is not part of the BASC's Internalizing Problems composite. Items that loaded at lower levels on this factor include the BRIEF's BRI and the BRI subscales of Shift and Emotional Control, and the BASC's BSI and Atypicality scale. The Perceptual Reasoning factor was composed entirely of items from the IQ instruments, including the Perceptual/Performance IQ composite score along with the associated subscales of Matrix Reasoning, Block Design, and Picture Concepts. The Adaptability factor was characterized by items from the BASC's Adaptive Skills composite, including Social Skills and Adaptability, and also by improved Attention scores from the BASC. The Processing Speed factor was characterized by the Processing Speed IQ Composite score and associated subscales of the WISC-IV/WPPSI-III, and the Verbal Intelligence factor was characterized by the Verbal IQ Composite score and the Verbal IQ Composite subscale of Vocabulary. In addition, Organization problems from the BRIEF also loaded onto the Verbal Intelligence factor; this seventh factor of Verbal Intelligence then is characterized not just by verbal intelligence but also by somewhat increased problems with organizational skills, although the loading for this item was low.

In sensitivity analyses we examined consistency of the factor structure by race (data not shown). The factor analysis was similar when conducted separately for whites and non-whites, 
with the exception of the seventh factor for verbal intelligence. This is likely because this factor only includes two items to characterize verbal intelligence - verbal IQ and vocabulary -- and thus has less capacity to explain variance. When we included items from the WISC-IV Verbal IQ composite that had been previously excluded (i.e., comprehension and similarities from the WISC-IV), then the seven factor solution in whites contained the same factors in both whites and non-whites, even though the total sample size dropped substantially because these analyses were limited to only white children who were administered the WISC-IV $(n=21)$. Finally, the factor structure was similar when using an oblique promax rotation (Appendix 2). Associations between smoking and neurodevelopment were similar when using promax rotated scores and the instrument-specific composite scores, but slightly different when using the varimax rotated scores (Appendix 3). This suggests the difference in results between the varimax rotated scores and the composite scores is due to the orthogonal rotation of the factors rather than the use of factor scores themselves.

\section{Early Life and Neurodevelopment Factors}

After accounting for the interrelationships among neurodevelopmental outcomes there were several notable associations (Table 6). The strongest associations for modifiable characteristics were for canned fish consumption, education, and preterm birth. Mothers who consumed canned fish at least once a week had children who scored half a standard deviation higher on the Perceptual Reasoning factor $(\hat{\beta} 0.50,95 \% \mathrm{Cl} 0.03,0.97)$. In contrast, children of mothers with a high school education or less had Verbal Intelligence factor scores approximately half a standard deviation lower than children of mothers with a higher education level $(\hat{\beta}-0.47,95 \% \mathrm{Cl}-0.78,-0.17)$. Preterm birth was also associated with more adverse Processing Speed $(\hat{\beta}-0.72,95 \% \mathrm{Cl}-1.31,-0.13)$, and Internalizing $\hat{(\beta}-0.62,95 \% \mathrm{Cl}-1.23,-0.02)$ factor scores. Of the HOME scores, only Organization displayed any associations with neurodevelopment, with a one tertile increase (corresponding approximately to slightly more 
than a one-point increase in the Organization score) resulting in a quarter of a standard deviation improvement in Executive Functioning $(\hat{\beta} 0.26,95 \% \mathrm{Cl} 0.04,0.49)$, and a small tenth of a standard deviation improvement in Adaptability factor scores $(\hat{\beta} 0.10,95 \% \mathrm{Cl} 0.00,0.19)$.

Of the non-modifiable characteristics, white race was associated with stronger Perceptual Reasoning and Verbal Intelligence scores (Perceptual Reasoning $\hat{\beta} 0.68,95 \% \mathrm{Cl}$ $0.25,1.10$; Verbal Intelligence $\hat{\beta} 0.81,95 \% \mathrm{Cl} 0.42,1.20)$, but was not associated with any other neurodevelopmental outcome. Girls averaged much higher Adaptability scores $(\hat{\beta} 0.54,95 \% \mathrm{Cl}$ $0.24,0.84)$ and higher Processing Speed scores $(\hat{\beta} 0.31,95 \% \mathrm{Cl} 0.00,0.62)$. Finally, larger head circumference at birth was associated with worse Executive Functioning factor scores $(\hat{\beta}$ 0.12, 95\% Cl -0.22, -0.01), but better Perceptual Reasoning $(\hat{\beta} 0.10,95 \% \mathrm{Cl} 0.00,0.19)$.

These associations were generally similar in the crude analyses, although some additional characteristics were found to have significant bivariate associations that were attenuated in multivariable adjusted models (Appendix 4). These multivariate regression associations also generally held in strata-specific analyses for whites and for non-whites, and were similar when additionally adjusting for maternal IQ, maternal marital status, and maternal age (data not shown).

As a case study, to compare associations across analysis methods, we examined the relationship of maternal prenatal smoking with neurodevelopment as measured by factor scores and the instrument-specific composite scores (Table 7). Of the 162 participants included in the analyses, 28 mothers reported any smoking during pregnancy, 17 of whom reported quitting before the second trimester.

Any smoking during pregnancy was associated with worse Impulsivity \& Externalizing factor scores in both methods assessed (Impulsivity \& Externalizing factor $\hat{\beta}-0.51,95 \% \mathrm{Cl}$ - 
0.92, -0.10; Externalizing composite $(\hat{\beta}-0.60,95 \% \mathrm{Cl}-1.00,-0.21)$. However, while smoking during pregnancy was not associated with worse Executive Functioning factor scores, it was associated with approximately $1 / 2$ a standard deviation worse Metacognition Index scores (Executive Functioning factor $\hat{\beta}-0.32,95 \% \mathrm{Cl}-0.72,0.10$; Metacognition Index $\hat{\beta}-0.47,95 \% \mathrm{Cl}$ $0.86,-0.09)$. This trend was more pronounced when examining associations between smoking in later pregnancy and associations with executive functioning (EF Factor $\hat{\beta}-0.32,95 \% \mathrm{Cl}-0.94$, 0.30; Metacognition Index $\hat{\beta}-0.55,95 \% \mathrm{Cl}-1.12,0.03)$. The smoking-neurodevelopment associations were generally stronger for participants who smoked during late pregnancy. While smoking in later pregnancy was negatively associated with the Adaptive Skills composite (Adaptive Skills composite $\hat{\beta}-0.66,95 \% \mathrm{Cl}-1.23,-0.10)$, the association was closer to the null for the corresponding factor (Adaptability factor $\hat{\beta}-0.27,95 \% \mathrm{Cl}-0.83,0.31$ ). Smoking was not associated with the Internalizing composite or Internalizing factor scores, or with any of the IQ composites or factor scores. Overall, accounting for the correlations among outcome measures by rotating factors to be linearly independent of one another resulted in attenuation of estimates for all but the Impulsivity and Externalizing factor, though all estimates were on the same side of the null.

\section{Discussion}

\section{Summary}

We identified seven factors that together captured $78 \%$ of the variation in a principal components analysis of the BASC, BRIEF, and WPPSI-III/WISC-IV in children between 6 and 9 years old. These factors were: 1) Impulsivity/Externalizing, 2) Executive Functioning, 3) Internalizing, 4) Perceptual Reasoning, 5) Adaptability, 6) Processing Speed, and 7) Verbal Intelligence. Although these factors roughly align with the composite indices of the included instruments, items from both the BASC and the BRIEF loaded onto all of the first three factors. 
This implies the existence of a meaningful correlational structure that is perhaps as strong across the BASC and the BRIEF as it is within-instrument: the Behavioral Regulation Index of the BRIEF and the Externalizing Index of the BASC appear to measure a similar underlying domain and load together, while the Metacognition Index of the BRIEF and the attention subscale from the BASC appear to measure another unique domain and load together.

Additionally, etiological interpretations were different for factor scores and composite scores. Although smoking in later pregnancy was associated with externalizing, executive functioning, and adaptive skills when using the composite scores, it was associated with only the Impulsivity \& Externalizing factor when using the orthogonal varimax rotated factor scores, which rotates factors to be linearly independent of one another. This implies that at least part of the association between smoking during pregnancy and the Metacognition Index and the Adaptive Skills Composite may be due to the correlation between those constructs and the Externalizing Composite. Accounting for such correlations by using factor scores may portray associations that are more accurate than using instrument-specific composites alone. Finally, the modifiable characteristics of education, canned fish consumption during pregnancy, preterm birth, and HOME Organization were associated with different neurodevelopmental factors.

\section{Factor Structure}

The factors to some extent aligned with measurement method. Specifically, scales from the BASC and the BRIEF loaded together without IQ items on four of the seven factors, while IQ items in the Processing Speed and Perceptual Reasoning factors loaded independently of the BASC and the BRIEF. The only exception was that organizational deficits from the BRIEF loaded positively on the Verbal Intelligence factor. Importantly, these clustering patterns also aligned with the measurement constructs of the instruments: the BRIEF and the BASC are both based on parent-report, while the WPPSI-III/WISC-IV are performance-based and assessed by research personnel. A limitation of the analysis is that we were unable to include multi-method 
measurements of the same neurodevelopmental outcome; for instance, our analysis included parent-report measures of executive functioning but lacked performance-based assessments of executive functioning. Previous factor analyses on performance-based and parent-report measures of executive functioning have shown that measurement method may explain more variance than the underlying domain (Bodnar, Prahme, Cutting, Denckla, \& Mahone, 2007; McAuley, Chen, Goos, Schachar, \& Crosbie, 2010). In those studies, parent-reported executive functioning and performance-based executive functioning loaded on separate factors, and the correlation between them was low, suggesting they measured different underlying features of executive functioning (Bodnar et al., 2007; McAuley et al., 2010). It is possible that this difference in measurement method drove the differential loadings of performance-based intelligence and parent-reported behavior and executive functioning in this analysis. Since factor structures of neurodevelopment vary according to the included instruments, specific phenotypes may vary across studies as different studies adopt different measurement tools. However, generalizable phenotypes may emerge from factor analyses of various instruments, such as the Impulsivity and Externalizing phenotype, which may help in interpreting etiologic associations across different studies.

\section{Early Life Characteristics and Neurodevelopmental Factors}

Several maternal and behavioral characteristics, as well as features of the home environment, were associated with factor scores after accounting for their interdependence. These associations were generally consistent with prior literature. Of the modifiable characteristics, there were particularly strong associations for consuming canned fish at least once a week during pregnancy and Perceptual Reasoning factor scores in childhood, higher maternal education and Verbal Intelligence factor scores, and preterm birth and Internalizing and Processing Speed factor scores, all of which are consistent with prior literature (Bhutta, Cleves, Casey, Cradock, \& Anand, 2002; Daniels, Longnecker, Rowland, Golding, \& ALSPAC, 
2004; S. Johnson \& Marlow, 2011; Oken et al., 2005; Sameroff, Seifer, Barocas, Zax, \& Greenspan, 1987). Although canned fish is often a source of other contaminants which have been associated with adverse neurodevelopment, such as PCBs and mercury (Gilbert \& GrantWebster, 1995), fish is also a source of other beneficial nutrients such as polyunsaturated fatty acids. Several studies of prenatal fish consumption support our findings that the benefits of fish consumption may outweigh the negative effects of environmental contamination (Cohen et al., 2005; Daniels et al., 2004; Hibbeln et al., 2007; Oken et al., 2005).

The only association that appeared inconsistent with prior findings was the relationship between head circumference at birth and Executive Functioning. Interestingly, while larger birth head circumference was associated with more deficits in Executive Functioning factor scores, it was also associated with better Perceptual Reasoning factor scores, in both unadjusted and adjusted analyses. While children with autism have larger head circumferences during childhood than average (Courchesne, Carper, \& Akshoomoff, 2003; Woodhouse et al., 1996), and have more problems with executive functioning (Christ, Holt, White, \& Green, 2007; Geurts, Verté, Oosterlaan, Roeyers, \& Sergeant, 2004), these children also have normal or smaller head circumferences at birth (Courchesne et al., 2001, 2003; Lainhart et al., 1997). In the animal literature, larger brain size across species has been associated with higher levels of inhibitory control (MacLean et al., 2014), although this ecological data does not necessarily apply within-species or at birth. This area of research is therefore relatively unexplored, and further research is necessary before drawing more definitive conclusions.

\section{Maternal Prenatal Smoking and Neurodevelopment}

Consistent with the prior literature, children of participants who reported smoking during later pregnancy had worse behavioral scores for the BASC's Externalizing composite, the BRIEF's Metacognition Index, and the BASC's Adaptive Skills composite, after adjustment for maternal education and race. However, after applying a rotational method to the factors that 
implemented statistical independence of the factors, only the association between smoking and the Impulsivity \& Externalizing factor remained in our study. The relationship between prenatal exposure to cigarettes and externalizing behaviors, ADHD, and executive functioning deficits in childhood has been frequently reported (Daseking, Petermann, Tischler, \& Waldmann, 2015; Langley, Rice, van den Bree, \& Thapar, 2005; Linnet et al., 2003; Piper \& Corbett, 2012). However, observed relationships between prenatal cigarette smoking and deficits in executive functioning may partially reflect associations between smoking and impulsivity and externalizing behaviors, due to the high correlation between these two constructs $\left(r_{s}\right.$ for the Externalizing composite and Metacognition Index in our population $=0.62$ ). While alternative methods may also be available to account for high correlation among outcomes (e.g., controlling for the correlated outcomes), applying orthogonal rotation techniques requires fewer degrees of freedom and is easily implemented while performing a factor analysis.

It should be noted that the reported association between smoking and Impulsivity and Externalizing may also be confounded by postnatal exposure to smoking and unmeasured maternal traits that increase both the propensity for smoking and risk of externalizing or executive functioning disorders (e.g., parental ADHD), which we were unable to control for (Thakur, Sengupta, Grizenko, Choudhry, \& Joober, 2012; Thapar et al., 2009). Although we report the strongest associations for maternal smoking in the second and third trimesters with Impulsivity and Externalizing factor scores, there are a number of potential caveats for these findings. Women who fail to quit smoking during pregnancy may smoke more per day and over a longer period. Children of women who smoked in later pregnancy may thus have a higher cumulative dose as well as a longer duration of exposure. Although the estimates for maternal first trimester smoking are not significant, they are in the same direction as the later pregnancy estimates. However, women who quit smoking before the second trimester may also have fewer problems with impulse control and addictive behaviors. Such characteristics may be either genetically or environmentally related to their children's propensity for such behaviors, and 
could confound associations between maternal smoking and childhood behavior. Additionally, the numbers of participants in each smoking category was relatively small; only 11 women reported any smoking in later pregnancy. However, the patterns of associations were similar when including all 28 women who reported any smoking during pregnancy, so the small numbers might not pose a significant threat.

\section{Strengths and Weaknesses}

A major strength of this analysis is the ability to examine multiple important characteristics and multiple neurodevelopmental outcomes while accounting for interdependencies among the covariates and the outcomes. The longitudinal, prospective nature of the original study allowed us to examine whether characteristics from the prenatal stage were associated with outcomes in later childhood. Dimension reduction enabled the simultaneous examination of a wide range of behaviors and cognitive outcomes, while taking advantage of correlational structures underlying different instruments. Accounting for the correlational structure among the factors may help clarify associations of specific characteristics with specific neurodevelopmental phenotypes, as we demonstrated in the case study of maternal smoking. Another advantage is the reduction in number of tests performed; examining associations with each subscale of each instrument would pose a larger threat from multiple testing. Finally, the principal components approach we employed presents conceptual advantages: factors that consolidate information across scales may provide a richer source of information than any single scale or composite. In etiological analyses, such factors may more closely mirror biological pathways that may be affected by prenatal and early life characteristics.

The most notable weakness is loss to follow-up; approximately $60 \%$ of the sample recruited at birth did not return for any follow-up assessment between the ages of 4-9 years. However, the distribution of the characteristics was mostly equivalent across these groups, so loss to follow-up was not influenced by any known covariates, with the possible exception of 
marital status. The loss to follow up did result in a reduced sample size, which may influence the quality of the factor analysis. Earlier recommendations for the necessary sample size for factor analysis range from at least 100 to at least 300 , although later studies have suggested that the sample size is less important as long as communalities are high and there are enough items with strong loadings on each factor (MacCallum et al., 1999). Our factor analysis had strong communalities and several items loaded highly on each factor, so the sample size of 210 for deriving the factor structure is likely adequate.

Another weakness is that although we were able to include important covariates in all models, only the models for smoking were built etiologically to examine associations with neurodevelopment. Depending on the "exposure" of interest, the associations in the general predictive models may include mediators or adjust for colliders, which may result in biased associations (Greenland, 2003; Kaufman, Maclehose, \& Kaufman, 2004).

A feature of the study that is both a strength and a limitation is that the study population was quite diverse and included both wealthy, mostly white mothers from the Upper East Side of Manhattan, and low-income, mostly minority mothers from East Harlem. Although these neighborhoods are adjacent, their socioeconomic features are widely divergent. In our analysis, these groups display little overlap with regards to socioeconomic characteristics. Regardless, the multivariate regression analyses remained similar for whites and non-whites, and the factor structure was similar for both whites and non-whites, suggesting such racial stratification may not be a significant threat.

In summary, we identified several maternal, birth, and home environment characteristics that were associated with neurodevelopmental phenotypes in linear regression analyses in a racially and socioeconomically diverse, urban cohort of mother-child pairs enrolled during pregnancy. We demonstrated that associations between smoking and correlated outcome domains may be substantially attenuated after accounting for their correlational structure. This "deep phenotyping" approach, that takes advantage of orthogonal rotation techniques, may 
more accurately represent associations with correlated neurodevelopmental domains than more traditional approaches that use instrument-specific composite scores. Phenotyping approaches may thus be useful in future etiological analyses of neurodevelopment in rich datasets with sufficient participants and measurements of multiple neurodevelopmental outcomes. 


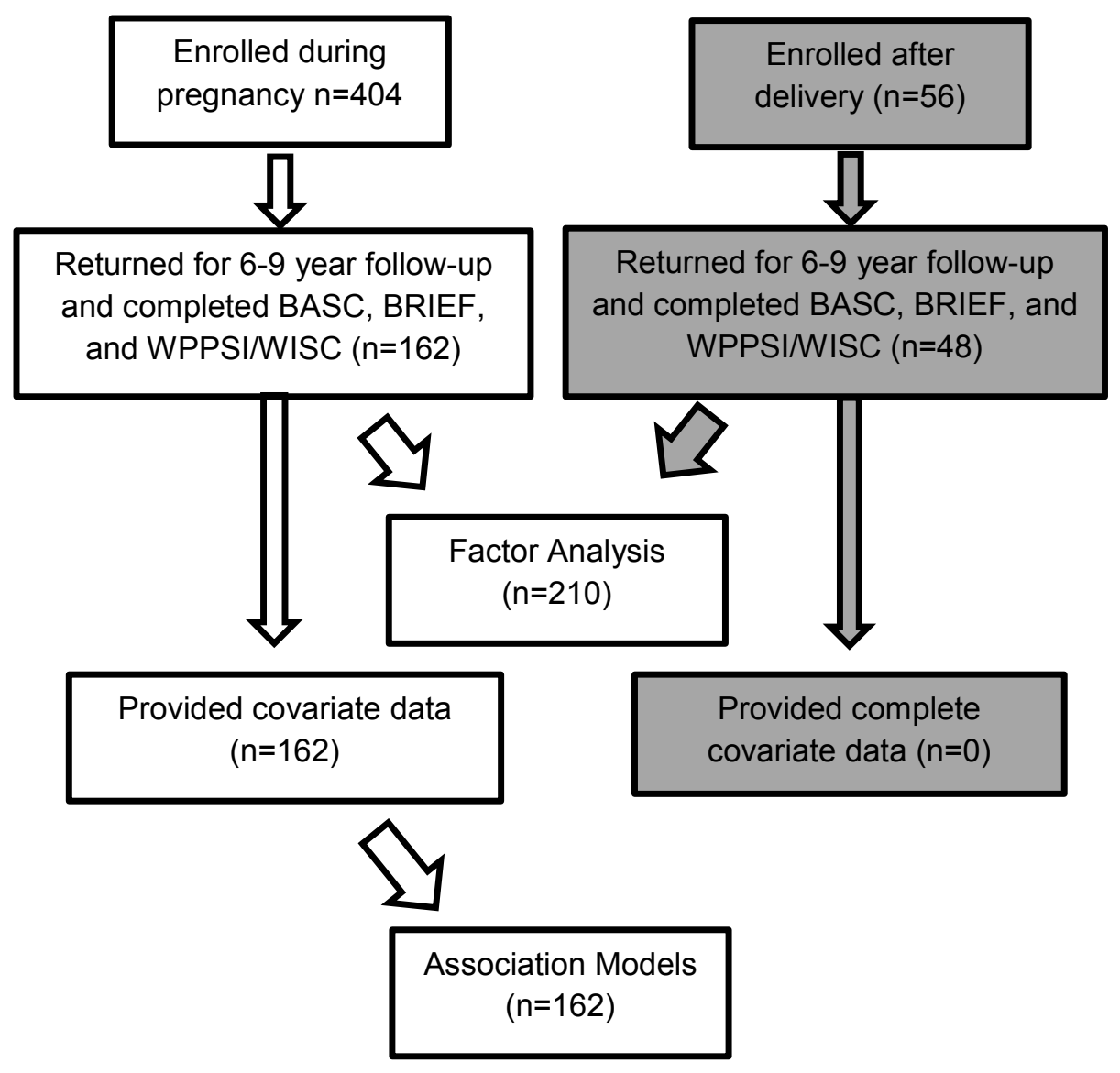

Figure 2 Origination and Follow-up of Participants Included in Factor Analysis and Regression Analyses 
Table 5 Varimax Rotated Factor Pattern Structure and Item Loadings of Childhood Neurodevelopmental Scales $(n=210)$

\begin{tabular}{|c|c|c|c|c|c|c|c|}
\hline \multicolumn{2}{|c|}{$\begin{array}{c}\text { Factor } 1 \\
\text { Impulsivity \& } \\
\text { Externalizing }\end{array}$} & \multicolumn{2}{|c|}{$\begin{array}{c}\text { Factor } 2 \\
\text { Executive Functioning }\end{array}$} & \multicolumn{2}{|c|}{$\begin{array}{l}\text { Factor } 3 \\
\text { Internalizing }\end{array}$} & \multicolumn{2}{|c|}{$\begin{array}{c}\text { Factor } 4 \\
\text { Perceptual Reasoning }\end{array}$} \\
\hline & Loadin & & Loadi & & Loadi & & Loadin \\
\hline $\begin{array}{l}\text { Scale } \\
\text { Externalizing }\end{array}$ & g & $\begin{array}{l}\text { Scale } \\
\text { Metacognition }\end{array}$ & $\mathrm{ng}$ & Scale & $\mathrm{ng}$ & $\begin{array}{l}\text { Scale } \\
\text { Perceptual }\end{array}$ & $g$ \\
\hline Problems $^{1}$ & 0.90 & Index 2 & 0.89 & Internalizing ${ }^{1}$ & 0.88 & $\begin{array}{l}\text { Reasoning } I^{3} \\
\text { Matrix }\end{array}$ & 0.97 \\
\hline Aggression $^{1}$ & 0.78 & $\begin{array}{l}\text { Planning } \\
\text { Global Exec }\end{array}$ & 0.83 & Anxiety $^{1}$ & 0.77 & Reasoning $^{3}$ & 0.76 \\
\hline Conduct $^{1}$ & 0.76 & $\begin{array}{l}\text { Composite }^{2} \\
\text { Working }\end{array}$ & 0.79 & Somatization ${ }^{1}$ & 0.67 & $\begin{array}{l}\text { Block Design }{ }^{3} \\
\text { Picture }\end{array}$ & 0.72 \\
\hline $\begin{array}{l}\text { Hyperactivity }{ }^{1} \\
\text { Behavioral Req }^{2}\end{array}$ & 0.74 & Memory $^{2}$ & 0.78 & Withdrawal $^{1}$ & 0.67 & Concepts $^{3}$ & 0.71 \\
\hline $\begin{array}{l}\text { Index } \\
\text { Behavioral }\end{array}$ & 0.69 & Monitor $^{2}$ & 0.77 & Depression $^{1}$ & 0.64 & Full Scale $I^{3}$ & 0.70 \\
\hline Symp Index ${ }^{1}$ & 0.68 & Initiate $^{2}$ & 0.77 & $\begin{array}{l}\text { Atypicality } \\
\text { Behavioral }\end{array}$ & 0.58 & Verbal IQ ${ }^{3}$ & 0.39 \\
\hline $\begin{array}{l}\text { Inhibit }^{2} \\
\text { Emotional }\end{array}$ & 0.66 & Organization ${ }^{2}$ & 0.62 & Symp Index ${ }^{1}$ & 0.53 & Vocabulary $^{3}$ & 0.31 \\
\hline Control $^{2}$ & 0.63 & Attention ${ }^{1}$ & 0.57 & $\begin{array}{l}\text { Shift }^{2} \\
\text { Emotional }\end{array}$ & 0.50 & & \\
\hline Adaptability $^{1}$ & -0.59 & $\begin{array}{l}\text { Inhibit }^{2} \\
\text { Behavioral }\end{array}$ & 0.56 & $\begin{array}{l}\text { Control }^{2} \\
\text { Behavioral }^{2}\end{array}$ & 0.44 & & \\
\hline $\begin{array}{l}\text { Shift }^{2} \\
\text { Global Exec }\end{array}$ & 0.51 & $\begin{array}{l}\text { Reg Index } \\
\text { Behavioral }\end{array}$ & 0.50 & $\begin{array}{l}\text { Reg Index } \\
\text { Global Exec }\end{array}$ & 0.38 & & \\
\hline Composite $^{2}$ & 0.49 & Symp Index ${ }^{1}$ & 0.42 & Composite $^{2}$ & 0.31 & & \\
\hline Depression $^{1}$ & 0.46 & Shift $^{2}$ & 0.42 & & & & \\
\hline Attention ${ }^{1}$ & 0.45 & $\begin{array}{l}\text { Hyperactivity } \\
\text { Emotional }\end{array}$ & 0.37 & & & & \\
\hline Monitor $^{2}$ & 0.41 & Control $^{2}$ & 0.35 & & & & \\
\hline Organization ${ }^{2}$ & 0.35 & Atypicality $^{7}$ & 0.30 & & & & \\
\hline Adaptive Skills ${ }^{1}$ & -0.33 & & & & & & \\
\hline Atypicality $^{1}$ & 0.30 & & & & & & \\
\hline Internalizing ${ }^{1}$ & 0.30 & & & & & & \\
\hline $\begin{array}{l}\text { Working } \\
\text { Memory }\end{array}$ & 0.3 & & & & & & \\
\hline $\begin{array}{l}\text { Factor } \\
\text { Structure } \\
\% \text { Variance }\end{array}$ & & & & & & & \\
\hline accounted for & 37.92 & & 13.71 & & 7.86 & & 6.33 \\
\hline Eigenvalue & 14.03 & & 5.07 & & 2.91 & & 2.34 \\
\hline
\end{tabular}

${ }^{1}$ BASC scales, ${ }^{2}$ BRIEF scales, ${ }^{3}$ WISC-IV or the WPPSI-III scales. Only items with loadings with absolute values $>0.30$ are shown here. Loadings are from a PCA with orthogonal varimax rotation (factors are uncorrelated). Positive loadings for items from the BRIEF and BASC all indicate more problems with those items, except for the BASC's Adaptive Skills, Social Skills, Leadership, and Adaptability. 
Table 5 (continued) Varimax Rotated Factor Pattern Structure and Item Loadings of Childhood Neurodevelopmental Scales $(n=210)$

\begin{tabular}{|c|c|c|c|c|c|}
\hline \multicolumn{2}{|c|}{$\begin{array}{c}\text { Factor } 5 \\
\text { Adaptability }\end{array}$} & \multicolumn{2}{|c|}{$\begin{array}{c}\text { Factor } 6 \\
\text { Processing Speed }\end{array}$} & \multicolumn{2}{|c|}{$\begin{array}{c}\text { Factor } 7 \\
\text { Verbal Intelligence }\end{array}$} \\
\hline $\begin{array}{l}\text { Scale } \\
\text { Adaptive }\end{array}$ & g & $\begin{array}{l}\text { Scale } \\
\text { Processing }\end{array}$ & Loading & Scale & Loading \\
\hline Skills Index ${ }^{1}$ & 0.89 & Speed Index ${ }^{3}$ & 0.96 & Vocabulary ${ }^{3}$ & 0.85 \\
\hline Social Skills ${ }^{1}$ & 0.85 & $\begin{array}{l}\text { Coding }^{3} \\
\text { Symbol }\end{array}$ & 0.88 & Verbal IQ ${ }^{3}$ & 0.83 \\
\hline Leadership $^{1}$ & 0.85 & Search $^{3}$ & 0.71 & Full Scale $\mathrm{IQ}^{3}$ & 0.57 \\
\hline $\begin{array}{l}\text { Adaptability }^{1} \\
\text { Attention }^{1} \\
\text { Initiate }^{1}\end{array}$ & $\begin{array}{r}0.56 \\
-0.36 \\
-0.30\end{array}$ & Full Scale $\mathrm{IQ}^{3}$ & 0.36 & $\begin{array}{l}\text { Organization } \\
\text { Symbol Search }^{3}\end{array}$ & $\begin{array}{l}0.38 \\
0.31\end{array}$ \\
\hline & 5.10 & & 4.25 & & 3.05 \\
\hline & 1.89 & & 1.57 & & 1.13 \\
\hline
\end{tabular}

${ }^{1}$ BASC scales, ${ }^{2}$ BRIEF scales, ${ }^{3}$ WISC-IV or the WPPSI-III scales. Only items with loadings with absolute values $>0.30$ are shown here. Loadings are from a PCA with orthogonal varimax rotation (factors are uncorrelated). Positive loadings for items from the BRIEF and BASC all indicate more problems with those items, except for the BASC's Adaptive Skills, Social Skills, Leadership, and Adaptability. 
Table 6 Covariate-Adjusted Linear Regression Associations Between Prenatal and Early Life Characteristics and Neurodevelopmental Factors In The Mount Sinai Children's Environmental Health Cohort $(n=162)$

\begin{tabular}{|c|c|c|c|c|c|c|c|}
\hline & $\begin{array}{c}\text { Factor } 1 \\
\text { Impulsivity } \\
\& \\
\text { Externalizi } \\
\text { ng } \\
\beta(95 \% \mathrm{Cl})\end{array}$ & $\begin{array}{c}\text { Factor } 2 \\
\text { Executive } \\
\text { Functioni } \\
\text { ng } \\
\beta(95 \% \\
\text { Cl) }\end{array}$ & $\begin{array}{c}\text { Factor } 3 \\
\text { Internali } \\
\text { zing } \\
\beta(95 \% \\
\mathrm{Cl})\end{array}$ & $\begin{array}{c}\text { Factor } 4 \\
\text { Perceptual } \\
\text { Reasoning } \\
\beta(95 \% \mathrm{Cl})\end{array}$ & $\begin{array}{c}\text { Factor } 5 \\
\text { Adaptabili } \\
\text { ty } \\
\beta(95 \% \\
\text { Cl) }\end{array}$ & $\begin{array}{c}\text { Factor } 6 \\
\text { Processin } \\
\text { g Speed } \\
\beta(95 \% \\
\text { Cl) }\end{array}$ & $\begin{array}{c}\text { Factor } 7 \\
\text { Verbal } \\
\text { Intelligen } \\
\text { ce } \\
\beta(95 \% \\
\text { Cl) }\end{array}$ \\
\hline \multicolumn{8}{|c|}{ Maternal Characteristics } \\
\hline $\begin{array}{l}\text { High School } \\
\text { or less }\end{array}$ & $\begin{array}{l}-0.11 \\
(-0.46 \\
0.24)\end{array}$ & $\begin{array}{l}0.24 \\
(-0.12 \\
0.60)\end{array}$ & $\begin{array}{l}-0.13 \\
(-0.49 \\
0.22)\end{array}$ & $\begin{array}{l}0.00 \\
(-0.33 \\
0.34)\end{array}$ & $\begin{array}{l}-0.15 \\
(-0.50 \\
0.19)\end{array}$ & $\begin{array}{l}0.25 \\
(-0.10 \\
0.60)\end{array}$ & $\begin{array}{l}-0.47 \\
(-0.78,- \\
0.17)\end{array}$ \\
\hline $\begin{array}{l}\text { Any college } \\
\text { or higher } \\
\text { Non- } \\
\text { Hispanic } \\
\text { White } \\
\text { Hispanic, }\end{array}$ & $\begin{array}{l}\text { referent } \\
-0.11 \\
(-0.55 \\
0.34)\end{array}$ & $\begin{array}{l}\text { referent } \\
-0.16 \\
(-0.62 \\
0.30)\end{array}$ & $\begin{array}{l}\text { referent } \\
-0.11 \\
(-0.57 \\
0.35)\end{array}$ & $\begin{array}{l}\text { referent } \\
0.68 \\
(0.25 \\
1.10) \\
\end{array}$ & $\begin{array}{l}\text { referent } \\
0.11 \\
(-0.33 \\
0.55)\end{array}$ & $\begin{array}{l}\text { referent } \\
-0.09 \\
(-0.54 \\
0.36)\end{array}$ & $\begin{array}{l}\text { referent } \\
0.81 \\
(0.42 \\
1.20) \\
\end{array}$ \\
\hline $\begin{array}{l}\text { Black, Other } \\
\text { Any alcohol } \\
\text { pregnancy }\end{array}$ & $\begin{array}{l}\text { referent } \\
0.02 \\
(-0.41 \\
0.45)\end{array}$ & $\begin{array}{l}\text { referent } \\
0.16 \\
(-0.28 \\
0.60)\end{array}$ & $\begin{array}{l}\text { referent } \\
0.16 \\
(-0.29 \\
0.60)\end{array}$ & $\begin{array}{l}\text { referent } \\
0.29 \\
(-0.12 \\
0.71)\end{array}$ & $\begin{array}{l}\text { referent } \\
0.00 \\
(-0.43 \\
0.43)\end{array}$ & $\begin{array}{l}\text { referent } \\
0.13 \\
(-0.31 \\
0.57)\end{array}$ & $\begin{array}{l}\text { referent } \\
0.34 \\
(-0.03 \\
0.71)\end{array}$ \\
\hline $\begin{array}{l}\text { No alcohol } \\
\text { pregnancy }\end{array}$ & referent & referent & referent & referent & referent & referent & referent \\
\hline $\begin{array}{l}\text { Canned fish } \\
\geq 1 \mathrm{x} / \text { week }\end{array}$ & $\begin{array}{l}0.04 \\
(-0.45 \\
0.53)\end{array}$ & $\begin{array}{l}-0.18 \\
(-0.69 \\
0.33)\end{array}$ & $\begin{array}{l}0.11 \\
(-0.39 \\
0.61)\end{array}$ & $\begin{array}{l}0.50 \\
(0.03 \\
0.97)\end{array}$ & $\begin{array}{l}0.28 \\
(-0.20 \\
0.77)\end{array}$ & $\begin{array}{l}-0.17 \\
(-0.66 \\
0.33)\end{array}$ & $\begin{array}{l}-0.09 \\
(-0.51 \\
0.34)\end{array}$ \\
\hline $\begin{array}{l}\text { Canned fish } \\
<1 \mathrm{x} / \text { week }\end{array}$ & referent & referent & referent & referent & referent & referent & referent \\
\hline \multicolumn{8}{|l|}{$\begin{array}{l}\text { Child and Birth } \\
\text { Characteristics }\end{array}$} \\
\hline Female & $\begin{array}{l}0.23 \\
(-0.08 \\
0.53)\end{array}$ & $\begin{array}{l}-0.15 \\
(-0.46 \\
0.17)\end{array}$ & $\begin{array}{l}-0.14 \\
(-0.46 \\
0.17)\end{array}$ & $\begin{array}{l}-0.04 \\
(-0.34 \\
0.25)\end{array}$ & $\begin{array}{l}0.54 \\
(0.24, \\
0.84)\end{array}$ & $\begin{array}{l}0.31 \\
(0.00 \\
0.62)\end{array}$ & $\begin{array}{l}-0.13 \\
(-0.39 \\
0.14)\end{array}$ \\
\hline $\begin{array}{l}\text { Male } \\
\text { Preterm } \\
\text { birth }\end{array}$ & $\begin{array}{l}\text { referent } \\
0.41 \\
(-0.18 \\
1.00)\end{array}$ & $\begin{array}{l}\text { referent } \\
-0.22 \\
(-0.82 \\
0.39)\end{array}$ & $\begin{array}{l}\text { referent } \\
-0.62 \\
(-1.23,- \\
0.02)\end{array}$ & $\begin{array}{l}\text { referent } \\
0.24 \\
(-0.32 \\
0.80)\end{array}$ & $\begin{array}{l}\text { referent } \\
0.32 \\
(-0.26 \\
0.90)\end{array}$ & $\begin{array}{l}\text { referent } \\
-0.72 \\
(-1.31,- \\
0.13)\end{array}$ & $\begin{array}{l}\text { referent } \\
0.32 \\
(-0.19 \\
0.83)\end{array}$ \\
\hline $\begin{array}{l}\text { Term birth } \\
\text { Birth Head } \\
\text { Circumferen } \\
\text { ce }(\mathrm{cm})\end{array}$ & $\begin{array}{l}\text { referent } \\
-0.03 \\
(-0.13 \\
0.08) \\
\end{array}$ & $\begin{array}{l}\text { referent } \\
-0.12 \\
(-0.22,- \\
0.01) \\
\end{array}$ & $\begin{array}{l}\text { referent } \\
0.01 \\
(-0.10 \\
0.11) \\
\end{array}$ & $\begin{array}{l}\text { referent } \\
0.10 \\
(0.00 \\
0.19) \\
\end{array}$ & $\begin{array}{l}\text { referent } \\
0.03 \\
(-0.07 \\
0.14) \\
\end{array}$ & $\begin{array}{l}\text { referent } \\
0.06 \\
(-0.05 \\
0.16) \\
\end{array}$ & $\begin{array}{l}\text { referent } \\
-0.01 \\
(-0.10 \\
0.08) \\
\end{array}$ \\
\hline \multicolumn{8}{|l|}{ HOME scores } \\
\hline $\begin{array}{l}\text { Organizatio } \\
\mathrm{n}\end{array}$ & $\begin{array}{l}-0.11 \\
(-0.32 \\
0.11)\end{array}$ & $\begin{array}{l}0.26 \\
(0.04 \\
0.49)\end{array}$ & $\begin{array}{l}0.20 \\
(-0.02 \\
0.43)\end{array}$ & $\begin{array}{l}-0.05 \\
(-0.26 \\
0.15)\end{array}$ & $\begin{array}{l}0.10 \\
(0.00 \\
0.19)\end{array}$ & $\begin{array}{l}-0.13 \\
(-0.35 \\
0.09)\end{array}$ & $\begin{array}{l}-0.01 \\
(-0.20 \\
0.17) \\
\end{array}$ \\
\hline $\begin{array}{l}\text { Learning } \\
\text { Materials }\end{array}$ & $\begin{array}{l}0.02 \\
(-0.21 \\
0.25) \\
0.06 \\
(-0.16 \\
0.27)\end{array}$ & $\begin{array}{l}0.02 \\
(-0.21 \\
0.26) \\
-0.17 \\
(-0.40 \\
0.06)\end{array}$ & $\begin{array}{l}0.01 \\
(-0.22 \\
0.25) \\
-0.07 \\
(-0.29 \\
0.15)\end{array}$ & $\begin{array}{l}0.06 \\
(-0.16 \\
0.27) \\
0.09 \\
(-0.12 \\
0.29)\end{array}$ & $\begin{array}{l}-0.05 \\
(-0.26 \\
0.15) \\
0.06 \\
(-0.16 \\
0.27)\end{array}$ & $\begin{array}{l}0.16 \\
(-0.07 \\
0.40) \\
0.09 \\
(-0.13 \\
0.31)\end{array}$ & $\begin{array}{l}0.12 \\
(-0.08 \\
0.32) \\
-0.03 \\
(-0.22 \\
0.16)\end{array}$ \\
\hline
\end{tabular}




\begin{tabular}{|c|c|c|c|c|c|c|c|}
\hline & $\begin{array}{c}\text { Factor } 1 \\
\text { Impulsivity } \\
\& \\
\text { Externalizi } \\
\text { ng } \\
\beta(95 \% \mathrm{Cl})\end{array}$ & $\begin{array}{c}\text { Factor } 2 \\
\text { Executive } \\
\text { Functioni } \\
\text { ng } \\
\beta(95 \% \\
\mathrm{Cl}) \\
\end{array}$ & $\begin{array}{c}\text { Factor } 3 \\
\text { Internali } \\
\text { zing } \\
\beta(95 \% \\
\mathrm{Cl})\end{array}$ & $\begin{array}{c}\text { Factor } 4 \\
\text { Perceptual } \\
\text { Reasoning } \\
\beta(95 \% \mathrm{Cl})\end{array}$ & $\begin{array}{c}\text { Factor } 5 \\
\text { Adaptabili } \\
\text { ty } \\
\beta(95 \% \\
\text { Cl) }\end{array}$ & $\begin{array}{c}\text { Factor } 6 \\
\text { Processin } \\
\text { g Speed } \\
\beta(95 \% \\
\mathrm{Cl})\end{array}$ & $\begin{array}{c}\text { Factor } 7 \\
\text { Verbal } \\
\text { Intelligen } \\
\text { ce } \\
\beta(95 \% \\
\mathrm{Cl}) \\
\end{array}$ \\
\hline Variety & $\begin{array}{l}-0.02 \\
(-0.25 \\
0.21)\end{array}$ & $\begin{array}{l}0.13 \\
(-0.11, \\
0.38)\end{array}$ & $\begin{array}{l}0.11 \\
(-0.13, \\
0.34)\end{array}$ & $\begin{array}{l}0.01 \\
(-0.22, \\
0.23)\end{array}$ & $\begin{array}{l}0.09 \\
(-0.12 \\
0.29)\end{array}$ & $\begin{array}{l}0.05 \\
(-0.19, \\
0.29)\end{array}$ & $\begin{array}{l}-0.07 \\
(-0.28 \\
0.13)\end{array}$ \\
\hline
\end{tabular}


Table 7 Comparison of Prenatal Smoking and Neurodevelopment Associations by Analysis Method $(n=162)$

\begin{tabular}{|c|c|c|c|}
\hline & $\begin{array}{l}\text { Any smoking during } \\
\text { pregnancy }\end{array}$ & $\begin{array}{l}\text { Smoking in First } \\
\text { Trimester Only }\end{array}$ & $\begin{array}{l}\text { Smoking in } \\
\text { Second or Third } \\
\text { Trimesters }\end{array}$ \\
\hline & $N=28$ & $\mathrm{~N}=17$ & $N=11$ \\
\hline $\begin{array}{l}\text { Impulsivity \& Externalizing } \\
\text { factor }^{1}\end{array}$ & $-0.51(-0.92,-0.10)$ & $-0.29(-0.80,0.22)$ & $-0.82(-1.42,-0.23)$ \\
\hline Externalizing Composite ${ }^{2}$ & $-0.60(-1.00,-0.21)$ & $-0.36(-0.84,0.13)$ & $-0.97(-1.55,-0.39)$ \\
\hline $\begin{array}{l}\text { Executive Functioning } \\
\text { factor }^{1}\end{array}$ & $-0.32(-0.75,0.10)$ & $-0.32(-0.85,0.21)$ & $-0.32(-0.94,0.30)$ \\
\hline Metacognition Index ${ }^{2}$ & $-0.47(-0.86,-0.09)$ & $-0.23(-0.71,0.25)$ & $-0.55(-1.12,0.03)$ \\
\hline Internalizing factor ${ }^{1}$ & $0.26(-0.16,0.68)$ & $0.32(-0.21,0.84)$ & $0.18(-0.43,0.80)$ \\
\hline Internalizing Composite ${ }^{2}$ & $0.07(-0.33,0.47)$ & $0.18(-0.32,0.68)$ & $-0.09(-0.68,0.50)$ \\
\hline $\begin{array}{l}\text { Perceptual Reasoning } \\
\text { factor }^{1}\end{array}$ & $0.06(-0.33,0.45)$ & $0.28(-0.21,0.77)$ & $-0.26(-0.83,0.31)$ \\
\hline Perceptual Reasoning IQ ${ }^{2}$ & $0.14(-0.25,0.53)$ & $0.37(-0.11,0.85)$ & $-0.19(-0.76,0.38)$ \\
\hline Adaptability factor ${ }^{1}$ & $-0.08(-0.48,0.33)$ & $0.06(-0.45,0.56)$ & $-0.27(-0.85,0.32)$ \\
\hline Adaptive Skills Composite ${ }^{2}$ & $-0.33(-0.71,0.06)$ & $-0.10(-0.58,0.37)$ & $-0.66(-1.23,-0.10)$ \\
\hline Processing Speed factor ${ }^{1}$ & $-0.16(-0.57,0.26)$ & $-0.09(-0.61,0.43)$ & $-0.26(-0.87,0.36)$ \\
\hline Processing Speed IQ ${ }^{2}$ & $-0.22(-0.66,0.23)$ & $-0.11(-0.69,0.48)$ & $-0.46(-1.07,0.15)$ \\
\hline Verbal Intelligence $^{1}$ & $0.04(-0.31,0.40)$ & $-0.12(-0.56,0.32)$ & $0.28(-0.23,0.79)$ \\
\hline Verbal IQ ${ }^{2}$ & $0.15(-0.16,0.46)$ & $0.10(-0.29,0.49)$ & $0.22(-0.24,0.68)$ \\
\hline
\end{tabular}

All models adjusted for maternal race, maternal education at follow-up, fish consumption, child sex, alcohol consumption during pregnancy, and HOME scores. Referent category for all three models is no reported smoking during pregnancy.

${ }^{1}$ Factors are rotated using Varimax (orthogonal) rotation (factors are statistically uncorrelated with each other).

${ }^{2}$ The composite/index items are the highest loading composite item for each factor. 


\section{CHAPTER V. ASSOCIATIONS BETWEEN PRENATAL EXPOSURE TO ORGANOPHOSPHORUS PESTICIDES AND CHILDHOOD NEURODEVELOPMENTAL PHENOTYPES}

\section{Introduction}

Prenatal exposure to organophosphorus pesticides (OPs), phthalates, and phenols have been associated with several aspects of risk to neurodevelopment in different populations (reviewed in (Bellinger, 2013)). However, epidemiological studies of OPs and neurodevelopment have typically considered only a single component of neurodevelopment at a time_for example, IQ or behavior. There are major conceptual advantages in jointly modeling domains of neurobehavioral development (Robinson, 2012). Accounting for interrelatedness between developmental domains is more clinically relevant, in part because several neurological functions rely on each other. For example, higher-level inhibitory control - typically considered to be a component of executive functioning -- relies on more basic processing speed capability, which is typically measured in intelligence tests (Ridderinkhof \& Van Der Molen, 1997). Additionally, deficits in one aspect of neural functioning may be compensated for by performance in other areas - for instance, declarative memory can compensate for multiple impairments across a range of disorders (Ullman \& Pullman, 2015). Finally, considering multiple aspects of neurodevelopment simultaneously allows the investigator to employ dimension reduction techniques, such as factor analysis, which enables the user to rotate factors to be linearly independent. This may account for any remaining interrelatedness among unique aspects of neurodevelopment in etiological studies.

By jointly considering behavior, cognition, and executive functioning, we may also better characterize patterns of deficits in neurodevelopment (Castellanos, Sonuga-Barke, Milham, \& Tannock, 2006; Mattison \& Mayes, 2012; Semrud-Clikeman, Walkowiak, Wilkinson, \& Butcher, 
2010; Sinzig, Morsch, Bruning, Schmidt, \& Lehmkuhl, 2008) that may provide insights into common etiological pathways. For instance, OPs can negatively influence serotoninergic processing (Aldridge, Meyer, Seidler, \& Slotkin, 2005; Aldridge, Seidler, \& Slotkin, 2004; Slotkin \& Seidler, 2005, 2008). Serotonin, in turn, can influence aggression, other problematic social behaviors, depression, and ADHD (Cadoret et al., 2003; Eley et al., 2004; Zoroğlu et al., 2002), the latter of which is characterized by impairments in executive functioning (Barkley, 1997). Animal and human studies do support that OPs may be associated with these outcomes (Bouchard, Bellinger, Wright, \& Weisskopf, 2010; Children et al., 2007; Furlong, Engel, Barr, \& Wolff, 2014; Middlemore-Risher, Buccafusco, \& Terry, 2010; Ricceri et al., 2003, 2006).

In addition, previous approaches to modeling associations between OPs and neurodevelopment have generally assumed that exposure to OPs and other chemicals is independent, and that such chemicals do not confound or alter the OP-neurodevelopment relationship. However, this modeling approach may bias estimates due to confounding by correlated xenobiotics. Chemicals may be correlated with each other due to similar sources, such as plasticizers in consumer products, insecticides for pest control, or multiple compounds found in food due to production or delivery practices or common dietary patterns (S. M. Engel \& Wolff, 2013). Epidemiological investigations of exposure mixtures are still in their infancy, and have typically focused on confounding by co-exposures within a class of compounds (e.g. within phthalates), rather than across classes of compounds. Bayesian hierarchical mixture models allow the stable estimation of effects in the presence of collinearity and a high number of covariates relative to sample size, through the use of prior assignment (Dunson, Herring, \& Engel, 2008; Herring, 2010)

In this study, we evaluate associations between OPs and behavior, executive functioning, and IQ after accounting for possible mixture effects with other co-exposures (specifically, phthalates, phenols, and pyrethroid pesticides), and implement a phenotypic approach to modeling neurodevelopment with a factor analysis. We also consider possible 
sources of heterogeneity in associations due to race, sex and genetic variants in PON1, a gene which is involved in the detoxification of OPs.

\section{Methods}

\section{Study Recruitment and Population}

The Mount Sinai Children's Environmental Health Center is a prospective cohort study of 404 mother infant-pairs from New York City. We recruited women during prenatal visits at either the Mount Sinai Diagnostic and Treatment Center, which serves a predominantly East Harlem population, or one of two private practices on the Upper East Side of Manhattan. Eligible mothers were primiparous with singleton pregnancies, and delivered at the Mount Sinai Hospital between May 1998 and July 2001 (Berkowitz et al., 2003, 2004). Exclusions have been detailed elsewhere (Berkowitz et al., 2003; S. M. Engel et al., 2007). Mothers completed questionnaires during their third trimester that assessed a variety of sociodemographic, behavioral, and medical history characteristics. We also obtained maternal spot urine samples between 25 and 40 weeks of gestation (mean= 31.2 weeks).

We invited participants to return for follow-up visits with their child at ages 1, 2, 4, 6, and 7-9 years. At follow-up visits, mothers completed questionnaires describing sociodemographic features and developmental milestones. The Home Observation for Measurement of the Environment (HOME scale) (Bradley et al., 1989) was administered in home visits at the 1 and 2 year follow-up visits. The HOME subscales include Involvement, Learning Materials,

Organization, Acceptance, Responsivity, and Variety (descriptions provided in Chapter 4).

\section{Exposure Biomarker Measurements}

The Centers for Disease Control (CDC) analyzed all maternal urine samples. Six dialkylphosphate metabolites, including three dimethylphosphate (DMP) and three diethylphosphate (DEP) metabolites, were analyzed in two batches between 2002-2003. Quality control and laboratory methods have been published previously (Barr et al., 2005; Bravo et al., 
2004). We excluded participants with very dilute urines (creatinine $<10 \mathrm{mg} / \mathrm{dL}$ ) due to the potential for inaccurate biomarker measurements (Wolff et al., 2008). After exclusion, 375 participants had at least one non-missing metabolite and 158 of these participants returned for a complete neurodevelopmental follow-up visit, had factor scores, and are included in this analysis.

Samples were also analyzed for 9 phthalate, 3 pyrethroid, and 5 phenol metabolites, using laboratory and quality control methods that have been described previously (Barr et al., 2010; Kato, Silva, Needham, \& Calafat, 2005; Ye, Kuklenyik, Needham, \& Calafat, 2005). Briefly, phthalates in urine were measured using automated sample preparation and an on-line solid-phase extraction method in conjunction with isotope dilution high-performance liquid chromatography/tandem mass spectrometry (SPE-HPLC-MS) (Kato et al., 2005). Urinary phenols were also measured using SPE-HPLC-MS (Ye et al., 2005). For the pyrethroids, an internal standard mixture of isotopically labeled 3PBA and trans-DCCA was used to spike $2 \mathrm{ml}$ of urine, which was then incubated with Beta-glucuronidase/sulfarase to liberate the conjugated metabolites. Hydrolysates were extracted with OASIS HLB mixed-mode solid-phase extraction cartridges, which were then washed with $5 \%$ methanol in a $0.1 \%$ acetic acid solution. Metabolites were eluted with methanol. HPLC/MS was used to analyze the extracts. Isotope dilution calibration was used to quantify 3PBA and trans-DCCA, while cis-DCCA was quantified using labeled trans-DCCA as an internal standard (Barr et al., 2010).

Child Behavior, Executive Functioning, Psychometric Intelligence Testing

We measured children's executive functioning and behavior at the 4, 6, and 7-9 year visits using parent report measures, and IQ at the 6 and 7-9 year visits using performancebased measures.

The Behavior Rating Inventory of Executive Functioning (BRIEF) is a parent-report assessment of children's problems with executive functioning over the past 6 months (Bodnar et 
al., 2007). Parents reported whether each behavior had been a problem on a 3 point scale, ranging from never, sometimes, or often. Validity studies report good reliability with high testretest reliability (mean $r_{\mathrm{s}}=0.81$ for parents across scales) and internal consistency (Cronbach's alphas range from $0.80-0.98$ across scales)(Gioia et al., 2000). Indices include the Behavioral Regulation Index and the Metacognition Index, both of which are age normed and combined to form the overall Global Executive Composite. Detailed descriptions of the indices and subscales are included in Table 4.

The Behavior Assessment System for Children (BASC) is a parent-report assessment of children's problem and adaptive behaviors in the home and community setting (Sandoval \& Echandia, 1994). Internal consistency reliability of this instrument is good (Cronbach's alphas average 0.80 across scales and ages), and test-retest reliabilities are also high (mean $r_{\mathrm{s}}=0.85$ for preschool, mean $r_{\mathrm{s}}=0.87$ for children ages 6-11) (Sandoval \& Echandia, 1994). Composite indices include Externalizing Behaviors, Internalizing Behaviors, Adaptive Skills, and the Behavioral Symptoms Index. Parents score individual items as happening or relevant Never, Sometimes, Often, Almost always, which are converted into scores of $0,1,2$, or 3 , and reflect increasing frequency. Scores are age-normed and reported as T-scores. Detailed descriptions of the composites and subscales are included in Table 4. The BASC and BRIEF were both completed at the 4, 6, and 7-9 year visits. We used the mean T-scores across all visits.

We administered the Wechsler Preschool and Primary Scales of Intelligence-III (WPPSIIII) at age 6 (mean age $=6.2, S D=0.2$ ), and the Wechsler Intelligence Scales-IV (WISC-IV) between the ages of $7-9$ years (mean age $=7.8, S D=0.8$ ). WISC-IV composite scores include the Verbal Comprehension score, the Perceptual Reasoning score, the Working Memory Index, and Processing Speed. Similarly, the WPPSI-III composites are corollaries of the WISC-IV composites and include Verbal Intelligence, Performance IQ (similar to Perceptual Reasoning), and Processing Speed, but not Working Memory. The WISC-IV and WPPSI-III are highly 
correlated $\left(r_{\mathrm{s}}=0.84\right.$ in our population); thus, if a child returned for both visits we used the WISCIV scores for all subtests.

\section{Statistical Methods}

We examined demographic characteristics of participants at enrollment by follow-up status. We used frequentist chi-square goodness-of-fit tests and alpha of 0.05 to assess if participants from the original birth cohort with OP biomarker data who did not return for followup differed from participants who had OP biomarker data and returned for a complete neurodevelopmental evaluation. Although we used follow-up characteristics in our association models, only characteristics at enrollment were available for participants who did not return for follow-up. Thus, we used characteristics at enrollment for these comparisons.

\section{Neurodevelopmental Factor Analysis and Outcomes}

We assessed neurodevelopmental factors by performing dimension reduction on the BRIEF, BASC, and WPPSI-III/WISC-IV instruments with a principal components analysis. Details have been described elsewhere (Furlong et al., n.d.). Briefly, we included composites and subscales in the factor analysis if they were available at both the 6 and 7-9 year visits. By using average scores across visits we were able to include participants who returned for different visits in a single factor analysis. For this analysis, we used a varimax rotation to ensure neurodevelopmental outcome factors were linearly independent. We standardized all factor scores to have a mean of 0 and a standard deviation of 1 . A beta coefficient of one can then be interpreted as an increase in one standard deviation of the factor score. Additionally, we scaled factors so that positive/adverse characteristics go in the same direction across factors in regression analyses, with positive scores indicating better outcomes and negative scores indicating more adverse outcomes. Additionally, since the WISC-IV Working Memory Index provides unique information on a performance-based metric of executive functioning, and could 
not be included in the factor analysis because it is only a component of the WISC-IV, we also included this index as an outcome in models.

In order to assess the utility of the factor analysis approach over a more traditional approach, we also conducted analyses of associations between the OPs and the individual items from the factors that displayed associations with the OPs.

\section{Coexposures}

Coexposures included phthalates, phenols, and pyrethroid pesticides. Four phthalate metabolites are metabolites of the same parent compound, Di(2-ethylhexyl) phthalate (DEHP) and were thus included as a micromolar sum of those metabolites (MEHP, MEHHP, MEOHP, and MECPP). MBzP, MCPP, MiBP and MBP were individually included. The pyrethroid metabolites 3PBA, c-DCCA, and t-DCCA displayed a low frequency of detection and were dichotomized to indicate concentrations above or below the LOD. The DCCA isomers and 3PBA were highly collinear; only five participants with detectable levels of c-DCCA or t-DCCA did not have detectable levels of 3PBA, so the DCCA metabolites were excluded from the analysis. Phenols included metabolites for BPA, BP3, triclosan, and 2,5 -DCP, and these individual metabolites were included as co-exposures. We excluded the phenol 2,4-DCP because it is a marker of the same parent compound as 2,5-DCP, but had a lower detection frequency. We standardized the natural log form of all continuous co-exposures to a mean of 0 and a standard deviation of 1.

\section{Bayesian Exposure Mixture Analyses}

In order to address potential issues of collinearity among metabolites and to stabilize models with large numbers of co-exposures and interactions, we used a Bayesian exposure mixture modeling framework. We employed hierarchical shrinkage techniques and specified priors for means and variances of beta coefficients for all variables. We assigned independent normal prior distributions with a mean of zero and a variance of $1 / \mathrm{T}^{2}$ (MacLehose, Dunson, 
Herring, \& Hoppin, 2007), where t equals 1 for all exposures and covariates (priors with mean of 0 and standard deviation of 1 ). In sensitivity analyses, prior distributions on beta coefficients for the OPs and co-exposures were specified such that individual metabolites within classes were shrunk towards a class mean; e.g., phthalate metabolites were shrunk towards a phthalate mean and each OP sum was shrunk towards the OP mean. The $\mathrm{t}$ for all metabolites within a class thus originated from the same class-specific distribution; in this analysis we used a uniform distribution from 0.001 to 5 for T (Gelman et al., 2013). The outcomes were specified with means originating from a normal distribution with a mean of 0 , and variances originating from a uniform distribution from 0 to 2. 10,000 burn-in samples were discarded and we used 50,000 iterations to arrive at the posterior estimates.

We imputed values below the limit of detection (<LOD) at each iteration of the Markov chain Monte Carlo algorithm from a truncated normal distribution with parameters defined as the mean and standard deviation of the underlying distribution, a lower bound of 0 , and an upper bound equal to the LOD (WinBUGS package djl.trunc.norm). If an OP metabolite was missing due to analytic interference, the missing value within a class was imputed based on the other non-missing values within that class, as has been described previously (S. M. Engel et al., 2007). Diethyl- and dimethyl-phosphate metabolites were then summed on a molar basis at each iteration of the MCMC algorithm to obtain total diethylphosphate ( $\Sigma \mathrm{DEPs})$ and total dimethylphosphate ( $\Sigma \mathrm{DMPs})$ biomarker concentrations.

Phthalate and phenol metabolite concentrations that were below the LOD were imputed as described above. The pyrethroid metabolite was dichotomized at the LOD in all models due to the low detection frequency in our population. All continuous co-exposures and outcomes were standardized to have a mean of 0 and a standard deviation of 1 . All continuous covariates were standardized to a mean of 0 and a standard deviation of 1 in order to facilitate the assignment of priors and comparisons of estimated effect sizes with dichotomous covariates, 
and to improve MCMC convergence (Gelman et al., 2013). Missing covariate data were imputed at each iteration of the MCMC algorithm under the assumption that covariates were missing at random. We assigned covariate estimates priors with a mean of 0 and standard deviation of 1 . Covariates with missing data included HOME scores, alcohol consumption during pregnancy, and maternal IQ. The HOME subscale scores were modeled as normally distributed random variables conditional on race, education, child sex, maternal IQ, smoking during pregnancy, canned fish consumption during pregnancy, the factor scores, marital status, and maternal age at enrollment. We modeled alcohol consumption during pregnancy using a logistic model conditional on race, education, canned fish consumption, smoking, the factor scores, marital status, maternal age at enrollment, maternal IQ, and HOME scores. Maternal IQ was modeled as a normally distributed random variable conditional on race, education, smoking during pregnancy, canned fish consumption, marital status, age at enrollment, HOME scores, and the factor scores. Models were run using WinBUGS14.

\section{Covariate Selection}

We constructed directed acyclic graphs (DAGs) for each factor, and considered the following variables for inclusion in the DAGs: maternal education at follow-up (high school or less, some college, or bachelor's degree), race/ethnicity (non-Hispanic white, Hispanic, black), maternal marital status at follow-up (single, living with a partner, married), maternal age, HOME environment (overall scores included as continuous, subscales included as ordinal categorical tertile variables due to their limited range), smoking during pregnancy (ever/never), alcohol use during pregnancy (none, light drinking $<3$ drinks on average per week during any trimester, moderate to heavy drinking of $\geq 3$ drinks on average per week during any trimester, child sex, canned fish consumption during pregnancy ( $<1$ time per week vs $\geq 1$ time per week during pregnancy), Spanish language spoken in home, OP analysis batch, and an indicator variable for 
examiner for the WISC-IV/WPPSI-III. We used the DAGs to identify and adjust for the minimally sufficient set for each factor. We adjusted for creatinine and OP analysis batch in all models

\section{Interaction Analyses}

We also assessed modification by race/ethnicity, sex, and maternal PON1 genotype status (PON1 Q192R and PON1 -108 C>T polymorphisms). The PON1 polymorphisms of -108 C>T and Q192R were dichotomized (-108: CC vs CT or TT; Q192R: QQ vs QR or RR). We considered interactions to be present if the $95 \%$ credible interval for the interaction term did not cross the null. Interactions between OPs and possible modifiers were assessed one at a time; i.e., we assessed interactions between $\Sigma$ DMPs and race separately from interactions between $\sum$ DEPs and race.

\section{Sensitivity Analyses}

In sensitivity analyses we employed a frequentist linear regression approach to assess overall associations between $\Sigma$ DEPs and $\Sigma$ DMPs and neurodevelopmental factors, with and without adjustment for co-exposures to phthalates, phenols and pyrethroids. If individual metabolite concentrations were below the limit of detection (LOD), a single random value <LOD was imputed from a log-normal distribution truncated at the LOD. Metabolites missing due to analytic interference were handled as above. Due to collinearity, inclusion of co-exposures was treated slightly differently in frequentist models. The phthalate metabolites of MiBP, MBP, and MEP are all members of a class of low molecular weight phthalates and thus were included as a molar sum, alongside DEHP and the individual metabolites MCPP and MBZP. The log forms of the variables were standardized to a mean of 0 and a standard deviation of 1 for comparability in frequentist models. We used multiple imputation to fill in missing covariate data (PROC GLM with PROC MI and PROC MIANALYZE) (10 imputations), and examined studentized residuals and leverages to identify potential outliers. We used SASV9.4 for frequentist analyses. All relevant code is included in Appendix 6. 


\section{Results}

Of the 404 mother/child pairs that participated in the original birth cohort, 162 returned for at least one complete neurodevelopmental follow-up visit when their child was between 6-9 years old. 158 of these women had prenatal OP biomarker data. Of the 158 women with complete neurodevelopmental data and OP biomarker data, 141 had complete co-exposure data and were included in the analysis. Participants included in this analysis were generally young (64\% under 25 at enrollment) and non-white (82\%). Most participants reported no alcohol consumption (83\%) and no smoking during pregnancy (84\%), and most had an educational attainment of high school or less at enrollment (73\%) (Table 8). Single marital status at enrollment was the only predictor of returning for follow-up $(p=0.03)$, and the distributions of education at enrollment, maternal age at delivery, race, alcohol, and smoking were generally similar for those included in this analysis compared to those who were not.

Individual OP metabolites varied in their frequency of detection, with dimethylthiophosphate (DMTP) displaying the highest frequency at $93.6 \%$ detects and diethyldithiophosphate (DEDP) displaying the lowest frequency at $16.7 \%$ detects (Table 9). Results were similar when including all 158 participants with OP biomarker data. Of the 158 participants with OP biomarker data, all had phthalate biomarkers, 153 had phenols, and 148 had pyrethroid data. 141 participants had a complete panel of biomarker data. Detection frequencies for the phthalates were high, ranging from $97.5 \%$ to $99.4 \%$ for the individual metabolites. Among the phenols, BP3 and 2,5-DCP were detected in every sample, while BPA was detected in $86.3 \%$ of samples and triclosan was detected in $79.7 \%$ of samples. The pyrethroid metabolite PBA had a much lower detection frequency and was only detected in $24.3 \%$ of samples. 


\section{Bayesian Exposure Mixture Modeling of Neurodevelopmental Factors}

After examining eigenvalues and parallel analysis results, we determined there was an optimal seven factor solution (Furlong et al., n.d.). The factors are 1) Impulsivity and Externalizing, 2) Executive Functioning, 3) Internalizing, 4) Perceptual Reasoning, 5) Adaptability, 6) Processing Speed, and 7) Verbal Intelligence (Table 5).

We examined studentized residuals and leverages and identified and excluded one outlier from both frequentist and Bayesian models with the highest residuals in several factor models. This participant had extremely low behavioral factor scores and exclusion of this observation changed effect estimates by more than $20 \%$.

Convergence diagnostics of the Bayesian models were excellent.

After adjustment for covariates and co-exposures, $\Sigma$ DMPs were unexpectedly

associated with better scores on the Executive Functioning factor $(\hat{\beta} 0.18,95 \% \mathrm{Cl} 0.04,0.31)$, which was also supported by nonsignificant results for the Working Memory Index $(\hat{\beta} 0.12,95 \%$ $\mathrm{Cl}-0.02,0.25)$ (Table 10). $\Sigma \mathrm{DMPs}$ were at the same time associated with more adverse Internalizing factor scores $(\hat{\beta}-0.13,95 \% \mathrm{Cl}-0.25,0.00)$. In contrast, $\Sigma$ DEPs were associated with more adverse scores on the WISC-IV's Working Memory Index $\hat{\beta}-0.17,95 \% \mathrm{Cl}-0.33$, 0.03), with no other notable associations among the individual factor scores. The magnitude of each of these associations is fairly small, representing less than a quarter of a standarddeviation change in the outcome per one standard deviation increase in exposure.

Associations of $\sum \mathrm{DMPs}$ with individual item scores within the Executive Functioning factor were generally similar, although there were no item-specific associations for the BRIEF's Organization, Shift, Behavioral Regulation Index, or Emotional Control scales, nor the BASC Behavioral Symptom Index, Hyperactivity or Atypicality scales. In contrast, the association between $\Sigma D M P s$ and items within the Internalizing factor was mostly restricted to the BASC'S 
Anxiety scale $(\hat{\beta}-0.14,95 \% \mathrm{Cl}-0.28,0.00)$, with no other associations among items in that factor (Table 11).

Heterogeneity in Associations by Race

There were no interactions between sex, race, or PON1 and the $\sum$ DMPs or $\sum$ DEPs, for any factor. However, there was an interaction between race and $\Sigma$ DMPs for the WISCIV's Working Memory Index. The positive association between LDMPs and the Working Memory Index was only present for blacks $(\hat{\beta} \quad 0.34,95 \% \mathrm{Cl} 0.15,0.53)$, but not for Hispanics $(\hat{\beta}-0.09$, $95 \% \mathrm{Cl}-0.27,0.08)$ nor whites $(\hat{\beta} \quad 0.16,95 \% \mathrm{Cl}-0.16,0.49), 95 \%$ credible interval did not cross the null).

\section{Sensitivity Analyses}

In sensitivity analyses we shrunk exposure estimates toward class means. Estimates were generally similar although all estimates were slightly closer to the null, as expected. We also examined associations using a frequentist, single exposure linear regression framework and overall found little difference in associations as compared to the Bayesian method, however effects were slightly more inflated and tended to be less precise, as expected. Directionality in associations, however, was generally conserved (Appendix 5).

\section{Discussion}

Using a Bayesian hierarchical approach, we report associations between $\sum$ DMPs and worse Internalizing factor scores. Among the items that comprise the Internalizing Factor, the inverse associations with $\sum$ DMPs appeared to be largely restricted to anxiety. Supporting associations between DMP parent pesticides and depression and anxiety, have been previously reported in murine models and one human study. Malathion induces anxiety and/or depressive behaviors in adult rats when administered in adulthood or in utero (Assini et al., 2005; Brocardo et al., 2007; Hashjin, Dizaj, Attaran, \& Koohi, 2013; N'Go et al., 2013). In humans, occupational 
exposure to malathion has also been associated with depression in adult farmers (Beard et al., 2014). Although there are no previously published findings of exposure to OPs in utero and internalizing, anxiety, or depressive symptoms in childhood, one study has reported an association between prenatal exposure to $\Sigma$ DMPs and Pervasive Developmental Disorder (PDD) at 24 months as measured by the Child Behavior Checklist (CBCL) (Children et al., 2007). The PDD designation in the CBCL includes some behaviors that are indicative of general internalizing psychopathology, such as avoiding eye contact and being unresponsive to affection, which is consistent with our findings.

We also found a relationship between $\sum$ DEPs and the Working Memory Index, which is consistent with other analyses within this cohort (S. Engel et al., 2011). however, our analytic approach enabled estimation with more precision. Several previous studies report associations between OPs and deficits in different domains of IQ, including the Working Memory Index, (Bouchard et al., 2011b; S. Engel et al., 2011; V. A. Rauh et al., 2006; V. Rauh et al., 2011), although we found inverse associations only for $\sum$ DEPs and the Working Memory Index.

Finally, we report an unexpected positive association between $\Sigma$ DMPs and the Executive Functioning factor, and supported by an elevated, but non-significant, association with the Working Memory Index. However, the association between $\Sigma$ DMPs and the Working Memory Index differed by race, with a positive association among blacks and no associations among whites or Hispanics.

In contrast to our findings, several previous studies have reported adverse associations between OPs and various measures of executive functioning, including attention, ADHD, and working memory (Bouchard et al., 2011a, 2010; Eskenazi et al., 2014; Marks et al., 2010; V. A. Rauh et al., 2006; V. Rauh et al., 2011; Yu et al., 2016), although studies examining prenatal exposure originate from only two other cohorts (Bouchard et al., 2011a; Marks et al., 2010; V. A. Rauh et al., 2006; V. Rauh et al., 2011). In the CHAMACOS cohort, Marks et al (Marks et al., 2010) reported associations between $\Sigma$ DMPs and $\Sigma$ DEPs and parent-report measures of 
attention problems at 3.5 but not 5 years, and also associations with a performance-based measure of attention, the Kiddie Connors Performance Test (K-CPT). $\Sigma$ DEPs, but not $\Sigma$ DMPs, were additionally associated with a psychometrician rating of ADHD symptoms in the same cohort of 5 year olds (Marks et al., 2010). Also in CHAMACOS, Bouchard et al (Bouchard et al., 2011b) reported strong associations between increasing prenatal levels of DMPs (but not DEPs) and worse performance on the Working Memory Index at seven years of age, along with other dimensions of IQ (Bouchard et al., 2011a). In the Columbia Center for Children's Environmental Health, Rauh et al report associations between prenatal chlorpyrifos exposure, which devolves into DEPs, and parent-report measures of ADHD problems and attention problems, at 3 years (V. A. Rauh et al., 2006). In the same cohort, Rauh et al reported associations between prenatal exposure to chlorpyrifos and more adverse performance on the Working Memory Index (V. Rauh et al., 2011). Therefore, the existing literature is limited and somewhat mixed. Our findings of an adverse association between prenatal DEP exposure and the Working Memory Index is supported by the studies from the Columbia Center for Children's Environmental Health, which are based on a population of women residing in New York City that were enrolled at the same time as our cohort. However, we also found a positive association between LDMPs and the Executive Functioning factor, which seemingly contradicts the associations reported in the CHAMACOS cohort. These inconsistencies may be attributed in part to the limitations of the DAP (dialkylphosphate; includes DMPs and DEPs) biomarkers as estimates of exposure to organophosphorus pesticides.

DAP biomarkers are non-specific, as the same metabolite is produced by multiple parent compounds, which may vary in their toxicities. The CHAMACOS cohort was recruited in an agricultural region of $\mathrm{CA}$ and over $80 \%$ of households had a farmworker that lived in the household during pregnancy. The participants in this cohort were likely exposed to a different constellation of pesticides than the urban Mount Sinai or Columbia populations. In a recent pooled analysis of these cohorts, significant heterogeneity was found in associations between 
¿DMPs and the Bayley Mental Development Index (MDI) by cohort. Associations between ¿DMPs and the MDI in CHAMACOS were substantially more deleterious than the three other included cohorts, and the authors argued that a specific agricultural pesticide used in the Salinas Valley that devolves into DMPs and was not experienced by the other urban cohorts, may partly explain this pattern (S. M. Engel et al., 2015). The exposure profile of our cohort is likely much more similar to the Columbia cohort, and thus their findings of an adverse association between chlorpyrifos and Working Memory are highly relevant. We are unable to compare our findings of improved executive functioning with increasing DMP exposure with the other cohorts, as the Columbia cohort measured only a chlorpyrifos-specific biomarker.

We did not find inverse associations between $\Sigma$ DEPs and the Executive Functioning factor, which was somewhat surprising since working memory is a component of executive functioning. Interestingly, the parent-report measure of the BRIEF (from which the majority of the Executive Functioning factor is derived) displays almost no correlation with the performancebased measures of executive functioning (the Working Memory Index) in our data $\left(r_{s}=0.09\right)$. This lack of correlation is consistently reported in the executive functioning measurement literature (reviewed in (Toplak, West, \& Stanovich, 2013)). In previous studies, parent-report and performance-based measures of executive functioning load on different factors, suggesting they measure different underlying features of executive functioning (Bodnar et al., 2007; McAuley et al., 2010). Our Executive Functioning factor, which is comprised entirely of parent-report features, may reflect a broad capacity to self-regulate and execute goal-directed behaviors in a home or community environment while coping with typical environmental distractions. In contrast, the Working Memory Index is measured by an external examiner whose goal it is to direct and keep a child on task in an environment that is relatively controlled and free of external distractions, and thus may reflect a child's working memory capacity only in this very specific context (Toplak et al., 2013). While the inconsistency between the two measures may caution interpretation of the factor's validity, in this same cohort, we previously reported an association 
between prenatal smoking and worse Executive Functioning factor scores in the expected direction (Furlong et al., n.d.). Since the smoking-executive functioning association is consistent with the literature, (Fried, Watkinson, \& Gray, 2003; Julvez et al., 2007; Piper \& Corbett, 2012) this serves as one positive control for the overall validity of this factor.

The heterogeneity in associations of DMPs with Working Memory by race suggests the presence of race-specific residual confounding in the association of DMPs. For instance, OP biomarkers (DAPs) measure metabolites of the toxic parent compounds, rather than exposure to the parent compounds themselves. The use of a urinary DAP biomarker when diet is a primary source of exposure has some limitations (Lu, Barr, Pearson, \& Waller, 2008; Wessels, Barr, \& Mendola, 2003). Participants may be exposed either to preformed metabolites on fruits and vegetables, or to the parent insecticide, which they subsequently metabolize. Preformed metabolites do not inhibit cholinesterase and are less toxic than parent compounds in this case (Duggan et al., 2003). In one study of OP exposure from fruits and vegetables, over half of samples obtained from fruits and vegetables were composed of more preformed metabolites than parent oxons or pesticides (Zhang, Driver, Li, Ross, \& Krieger, 2008). Thus, measured urinary DAP biomarker levels may not always accurately reflect extent of exposure to the parent compound. If fruit and vegetable consumption is either directly associated with improved executive functioning or is a marker of generally healthier behaviors, exposure source could be an important confounder. Little has been published on direct associations between prenatal fruit and vegetable consumption and cognitive development, although one recent study showed improved cognitive outcomes at 1 year for children of mothers who consumed high amounts of fruit and fruit juice during pregnancy (Bolduc et al., 2016), despite also being a likely vector for pesticide exposure.

Additionally, executive functioning is highly heritable (Friedman et al., 2008). Mothers with robust executive functioning may be more successful at planning and consuming a wellbalanced diet that is rich in fruits and vegetables, and the use of the DAP biomarkers may be 
less accurate at measuring toxicity in these women. Women with higher executive functioning may also be more likely to return for follow-up. Thus, for these women, DAP biomarkers might be a surrogate for higher executive functioning. Although we did have maternal IQ, the correlation between Full Scale IQ and executive functioning as measured by the BRIEF in our data and in the literature is close to zero; thus this residual confounder was truly unmeasured. Finally, women with high biomarker levels of OPs in New York City may live in close proximity to outdoor areas such as parks, which may be sprayed for mosquito control. Their children may experience more aerobic exercise and engage in more play experiences with other children, which have been associated with improvements in executive functioning (reviewed in (Diamond \& Lee, 2011)). In these instances, OP biomarkers would be confounded by early life experiences that enhance executive functioning.

The literature on OP-PON1 interactions is sparse. While some studies have also reported no interactions with PON1 and OPs on mental development at 24 months (Eskenazi et al., 2010), recently a pooled study of four different birth cohorts showed stronger associations between $\sum$ DEPs and worse mental development at 24 months among carriers of the PON1 Q allele (S. M. Engel et al., 2015). A previous study in our population reported adverse associations between $\Sigma \mathrm{DMPs}$ and perceptual reasoning among those with the PON192 QQ genotype (S. Engel et al., 2011). While the strata-specific estimates for PON1 interactions in this current study were all highly imprecise and all interaction p-values were greater than 0.05 , the estimate for $\sum$ DMPs and the Perceptual Reasoning factor remained negative among those with the QQ genotype. Although PON1 is an important component in the detoxification pathway for OP and other pesticides, this enzyme may have limited capacity to hydrolyze OP pesticides with dimethyl parent oxons (Geldmacher-von Mallinckrodt \& Diepgen, 1988; Li et al., 2000; Wolff et al., 2007), which may in part explain the general lack of interactions between DMPs and PON1 genotype. 


\section{Strengths and Limitations of Study}

Primary strengths of this study include the incorporation of mixture modeling techniques for the exposures, and the use of dimension reduction across multiple scales and subscales of behavior, executive functioning, and intelligence. The use of factor scores resulted in reduced dimensionality of the outcomes and enabled comparison of risk estimates across a wide range of outcomes in an easily interpretable format. Factor scores may capture information over and above individual item scores. Although some items in different factors were correlated with each other (e.g., the Metacognition Index from the Executive Functioning factor and the Internalizing Composite score from the Internalizing factor are correlated at $\sim 0.5$ ), the rotated factor scores are forced to be orthogonal. Thus, associations with the factor scores are independent of any relationship between OPs and the other outcomes. The same is not true when interpreting associations with the individual items that make up these factors. An attempt to assess associations with all scales and subscales of several instruments in the absence of dimension reduction would present challenges to interpretation, and raise concerns about multiple testing bias.

Primary limitations include loss to follow-up and the lack of corroborating data on parent pesticide exposure. Approximately $60 \%$ of the original cohort did not return for follow-up. However, the only characteristic that differed between those who did and did not return was marital status, which was included as a covariate in several models. Still, if other unknown covariates predicted follow-up, these could bias associations. For instance, if more health conscious mothers consume more fresh fruits and vegetables and are more likely to return for a follow-up visit, they would be expected to have higher urinary OP metabolite levels and might also have children with better behavior and intelligence scores. We lacked data on such attitudes among study participants. Finally, urinary metabolites are an imperfect surrogate of insecticide exposure. Individuals who consume high levels of fresh fruits and vegetables may show higher urinary levels of ingested preformed OP metabolites, but experience less adverse 
effects, than individuals whose primary exposure is to the more toxic parent compound, which they subsequently metabolize.

Conclusions

In this prospective study of in utero exposure to organophosphorus pesticides, we report adverse associations between LDMPs and Internalizing Factor scores, which was somewhat restricted to an item-specific association with anxiety, and between $\sum$ DEPs and the Working Memory Index. We also report an unexpected positive association between $\Sigma$ DMPs and the Executive Functioning factor. Dimension reduction across multiple scales of neurodevelopment reflects broad phenotypes of development, eases presentation of associations, and incorporates information across multiple scales to enhance precision of estimates. 
Table 8 Characteristics of Mount Sinai Children's Environmental Health Center Study Population by Follow Up Status, For Those with Complete Panel of Biomarkers

\begin{tabular}{|c|c|c|c|}
\hline & $\begin{array}{c}\text { Original } \\
\text { Birth cohort } \\
N=404\end{array}$ & $\begin{array}{l}\text { Did not } \\
\text { return for } \\
\text { Follow-up } \\
n=263\end{array}$ & $\begin{array}{c}\text { Follow-up } \\
\text { Population N (\%) } \\
\mathrm{N}=141\end{array}$ \\
\hline \multicolumn{4}{|l|}{$\begin{array}{l}\text { Maternal Marital Status at } \\
\text { Enrollment }\end{array}$} \\
\hline Married & $117(29)$ & $83(32)$ & $34(24)$ \\
\hline Living with partner & $98(24)$ & $68(26)$ & $30(21)$ \\
\hline Single/Divorced/ Widowed & $189(47)$ & $112(43)$ & $77(55)$ \\
\hline \multicolumn{4}{|l|}{ Maternal Education at Enrollment } \\
\hline High school or less & $288(72)$ & $186(71)$ & $102(73)$ \\
\hline Some college or higher & $113(28)$ & $75(29)$ & $38(27)$ \\
\hline \multicolumn{4}{|l|}{ Maternal Age at enrollment } \\
\hline$<20$ & $142(35)$ & $101(38)$ & $41(29)$ \\
\hline $20-25$ & $132(33)$ & $83(32)$ & $49(35)$ \\
\hline$>25$ & $130(32)$ & $79(30)$ & $51(36)$ \\
\hline \multicolumn{4}{|l|}{ Maternal Race } \\
\hline Black or other race & $118(29)$ & $76(29)$ & $42(30)$ \\
\hline White & $86(21)$ & $60(23)$ & $26(18)$ \\
\hline Hispanic & $200(50)$ & $127(48)$ & $73(52)$ \\
\hline \multicolumn{4}{|l|}{ Any smoking during pregnancy } \\
\hline None & 337 (83) & $218(83)$ & $119(84)$ \\
\hline Any & $67(17)$ & $45(17)$ & $22(16)$ \\
\hline \multicolumn{4}{|l|}{ Alcohol use during pregnancy } \\
\hline None & $337(85)$ & $222(86)$ & $115(83)$ \\
\hline Light & $49(12)$ & $30(12)$ & $19(14)$ \\
\hline Moderate & $10(3)$ & $6(2)$ & $4(3)$ \\
\hline \multicolumn{4}{|l|}{$\begin{array}{l}\text { Canned fish consumption during } \\
\text { pregnancy }\end{array}$} \\
\hline$<1$ times per week & $341(84)$ & $218(83)$ & $123(87)$ \\
\hline 1 or more times per week & $63(16)$ & $45(17)$ & $18(13)$ \\
\hline \multicolumn{4}{|l|}{ Child Sex } \\
\hline Male & $220(54)$ & $148(56)$ & $72(51)$ \\
\hline Female & $184(46)$ & $115(44)$ & $69(49)$ \\
\hline
\end{tabular}

†Maternal marital status at enrollment differed for those with biomarker data who returned for follow-up vs those who didn't $(p=0.03)$. No other enrollment characteristics differed by follow-up status.

Follow-up population includes those with complete biomarker data and a complete neurodevelopmental follow-up visit. Comparison population includes those without biomarker data or those who did not return for a complete follow-up visit 
Table 9 Distributions of Organophosphorus Pesticide Metabolites and Co-exposures, Among Participants with Complete Follow-Up Measurements

\begin{tabular}{|c|c|c|c|c|c|c|}
\hline & & $\mathrm{N}$ & $\begin{array}{l}\text { Values } \\
>\text { LOD } \\
\text { (\% of } \\
\text { total) }\end{array}$ & $\begin{array}{l}\text { Geometric } \\
\text { Median } \\
(G S D)\end{array}$ & $\begin{array}{l}\text { Geometric } \\
25,75 \\
\text { percentile }\end{array}$ & Min, Max \\
\hline \multicolumn{7}{|c|}{ Organophosphorus Pesticides (nm/L) } \\
\hline \multirow[t]{4}{*}{$\Sigma \mathrm{DEP}$} & Diethylthiophosphate (DETP) & 156 & $\begin{array}{l}127 \\
(81.4)\end{array}$ & $10.0(3.5)$ & $4.9,17.0$ & $0.8,122.3$ \\
\hline & $\begin{array}{l}\text { Diethyldithiophosphate } \\
\text { (DEDP) }\end{array}$ & 156 & $\begin{array}{l}26 \\
(16.7)\end{array}$ & $0.1(4.5)$ & $0.1,0.1$ & 0.0 .59 .9 \\
\hline & Diethylphosphate (DEP) & 156 & $\begin{array}{l}72 \\
(46.2)\end{array}$ & $0.2(15.1)$ & $0.2,31.5$ & $0.2,371.8$ \\
\hline & $\Sigma \mathrm{DEP}$ & 156 & $\begin{array}{l}138 \\
(88.5)^{*}\end{array}$ & $17.5(3.2)$ & $7.3,47.1$ & $2.7,422.3$ \\
\hline \multirow[t]{4}{*}{$\Sigma \mathrm{DMP}$} & $\begin{array}{l}\text { Dimethylthiophosphate } \\
\text { (DMTP) }\end{array}$ & 158 & $\begin{array}{l}146 \\
(93.6)\end{array}$ & $23.8(6.2)$ & $8.1,71.9$ & $0.2,4754.3$ \\
\hline & $\begin{array}{l}\text { Dimethyldithiophosphate } \\
\text { (DMDP) }\end{array}$ & 158 & $\begin{array}{l}47 \\
(30.1)\end{array}$ & $0.3(6.2)$ & $0.3,5.1$ & 0.3 .275 .1 \\
\hline & Dimethylphosphate (DMP) & 158 & $\begin{array}{l}102 \\
(64.6)\end{array}$ & $13.6(8.7)$ & $0.6,45.0$ & $0.6,1173.1$ \\
\hline & $\sum \mathrm{DMP}$ & 158 & $\begin{array}{l}149 \\
(94.3)^{*}\end{array}$ & $41.2(5.2)$ & $\begin{array}{l}12.2 \\
148.5\end{array}$ & $0.2,4903.2$ \\
\hline \multicolumn{7}{|c|}{ Phthalates $(\mathrm{ng} / \mathrm{mL})$} \\
\hline & $\Sigma \mathrm{DEHP}(\mu \mathrm{m} / \mathrm{L})^{*}$ & 158 & $\begin{array}{l}158 \\
(100.0)^{*}\end{array}$ & $0.3(3.6)$ & $0.1,0.5$ & $0.0,19.9$ \\
\hline & Low- MWP* $(\mu \mathrm{m} / \mathrm{L})^{*}$ & 158 & $\begin{array}{l}158 \\
(100.0)^{*}\end{array}$ & $2.5(4.4)$ & $1.0,6.5$ & $0.0,313.8$ \\
\hline & Monoethyl phthalate (MEP) & 158 & $\begin{array}{l}157 \\
(99.4)\end{array}$ & $\begin{array}{l}217.0 \\
(4.2)\end{array}$ & $\begin{array}{l}84.9 \\
532.5\end{array}$ & $6.5,29528.4$ \\
\hline & $\begin{array}{l}\text { Mono-n-butyl phthalate } \\
\text { (MBP) }\end{array}$ & 158 & $\begin{array}{l}154 \\
(97.5)\end{array}$ & $34.0(3.6)$ & $14.3,83.3$ & $0.8,4042.5$ \\
\hline & $\begin{array}{l}\text { Monoisobutyl phthalate } \\
\text { (MIBP) }\end{array}$ & 158 & $\begin{array}{l}155 \\
(98.1)\end{array}$ & $6.5(2.9)$ & $3.1,15.1$ & $0.4,65.1$ \\
\hline & $\begin{array}{l}\text { Mono(3-carboxypropyl) } \\
\text { phthalate (MCPP) }\end{array}$ & 158 & $\begin{array}{l}154 \\
(97.5)\end{array}$ & $3.1(2.8)$ & $1.7,6.4$ & $0.3,129.3$ \\
\hline & $\begin{array}{l}\text { Monobenzyl phthalate } \\
\text { (MBZP) }\end{array}$ & 158 & $\begin{array}{l}157 \\
(99.4)\end{array}$ & $15.8(3.9)$ & $6.3,34.1$ & $0.6,481.3$ \\
\hline \multicolumn{7}{|c|}{ Phenols (ng/mL) } \\
\hline & Biphenyl-A (BPA) & 153 & $\begin{array}{l}132 \\
(86.3)\end{array}$ & $1.5(2.4)$ & $0.9,2.5$ & $0.4,35.2$ \\
\hline & Benzophenone-3 (BP3) & 153 & $\begin{array}{l}153 \\
(100.0)\end{array}$ & $6.5(7.4)$ & $2.7,23.7$ & $0.2,9290.0$ \\
\hline & 2,5-Dichlorphenol (2,5-DCP) & 153 & $\begin{array}{l}153 \\
(100.0)\end{array}$ & $56.3(5.5)$ & $\begin{array}{l}22.4 \\
174.0\end{array}$ & 2.8. 8510.0 \\
\hline & Triclosan & 153 & $\begin{array}{l}122 \\
(79.7)\end{array}$ & $20.0(5.2)$ & $7.2,82.8$ & $2.3,1790.0$ \\
\hline \multicolumn{7}{|c|}{ Pyrethroids (binary for above or below the LOD) } \\
\hline
\end{tabular}




\begin{tabular}{|lll}
\hline 3-PBA & $148 \quad 36$
\end{tabular}

${ }^{*}$ Values $>$ LOD for sum values indicate number of observations where at least one metabolite in the sum is above the LOD

Low molecular weight phthalates include MEP, MBP, MIBP

Values presented are after imputing values below the LOD from a normal distribution truncated at the LOD

141 participants had a complete panel of biomarker data 
Table 10 Overall Associations between OPs and Neurodevelopmental Factor Scores $(n=141)$

\begin{tabular}{|c|c|c|}
\hline & $\begin{array}{c}\sum \text { DMPs } \hat{\beta}(95 \% \\
\mathrm{Cl})^{¥}\end{array}$ & $\sum$ DEPs $\underset{¥}{\widehat{\beta}}(95 \% \mathrm{Cl})$ \\
\hline Impulsivity \& Externalizing Factor & $-0.02(-0.16,0.11)$ & $0.08(-0.06,0.22)$ \\
\hline Executive Functioning Factor & $0.18(0.04,0.31)$ & $-0.05(-0.21,0.11)$ \\
\hline Internalizing Factor & $-0.13(-0.25,0.00)$ & $-0.01(-0.15,0.12)$ \\
\hline Perceptual Reasoning Factor & $0.00(-0.14,0.13)$ & $-0.02(-0.17,0.14)$ \\
\hline Adaptability Factor & $-0.02(-0.14,0.12)$ & $-0.08(-0.23,0.07)$ \\
\hline Processing Speed Factor & $-0.01(-0.14,0.13)$ & $0.03(-0.12,0.19)$ \\
\hline Verbal Intelligence Factor & $0.07(-0.04,0.19)$ & $-0.03(-0.17,0.09)$ \\
\hline Working Memory Index & $0.12(-0.02,0.25)$ & $-0.17(-0.33,-0.03)$ \\
\hline
\end{tabular}

FTese Bayesian beta coefficients and 95\% credible intervals are from models that include both $\Sigma$ DAP measures ( $\Sigma D E P s$ and $\Sigma D M P s)$, the molar sum of DEHP and the individual phthalate congeners of MEP, MIBP, MBP, MCPP, MBZP, as well as the individual phenol metabolites BPA, BP3, DCP25, and triclosan, and the pyrethroid metabolite 3PBA

In Tables 10 and 11, scores have been scaled so that positive scores indicate more positive outcomes and negative scores indicate more adverse outcomes HOME scores, maternal IQ, maternal education at follow-up, maternal marital status at follow-up, maternal age, maternal race/ethnicity, maternal smoking during pregnancy, maternal alcohol consumption during pregnancy, maternal canned fish consumption during pregnancy, and alcohol were included in a DAG used to derive minimally sufficient sets for each factor 


\section{Table 11 Associations between DMPs and Individual Factor Items from Executive Functioning and Internalizing Factors $(n=141)$}

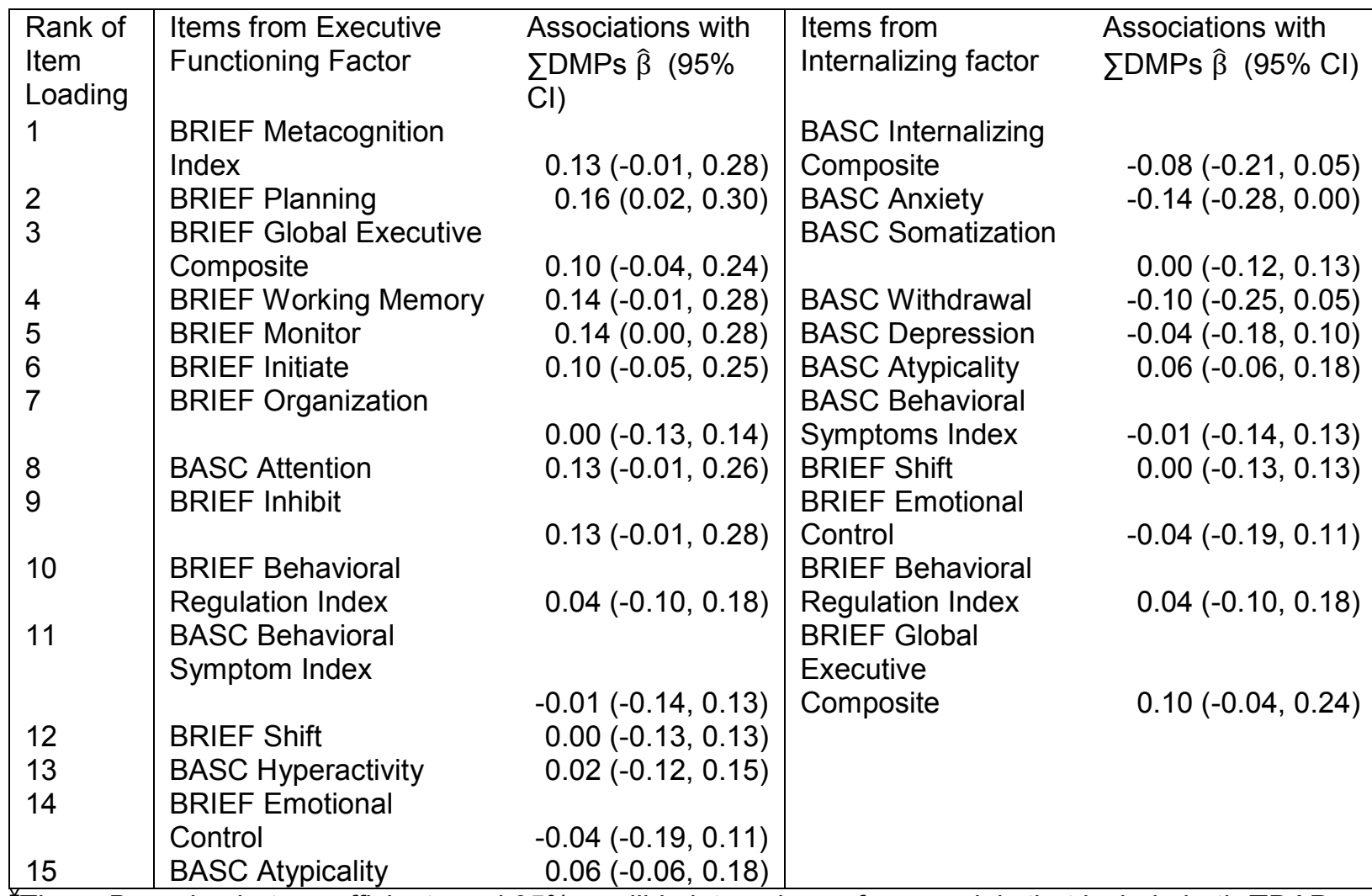

FThese Bayesian beta coefficients and $95 \%$ credible intervals are from models that include both $\sum$ DAP measures ( $\Sigma D E P s$ and $\Sigma D M P s$ ), the molar sum of DEHP and the individual phthalate congeners of MEP, MIBP, MBP, MCPP, MBZP, as well as the individual phenol metabolites BPA, BP3, DCP25, and triclosan, and the pyrethroid metabolite 3PBA

In Tables 10 and 11, scores have been scaled so that positive scores indicate more positive outcomes and negative scores indicate more adverse outcomes

HOME scores, maternal IQ, maternal education at follow-up, maternal marital status at follow-up, maternal age, maternal race/ethnicity, maternal smoking during pregnancy, maternal alcohol consumption during pregnancy, maternal canned fish consumption during pregnancy, and alcohol were included in a DAG used to derive minimally sufficient sets for each factor 


\section{CHAPTER VI. CONCLUSIONS}

\section{Summary of Findings}

Previous approaches to etiological studies of organophosphorus pesticides (OPs) and neurodevelopment have generally been performed under the assumptions that neurological traits are independent and that confounding by other xenobiotics is negligible. In this dissertation, we estimated associations between OPs and neurodevelopment while addressing both of these concerns.

Factor analysis is a dimension reduction method which uses underlying correlational structures to derive factors, and allows the user to rotate unique factors to be orthogonal. We applied this method to a set of composite scales and subscales originating from both parentreport measures of behavior and executive functioning, and performance-based measures of intelligence. We determined there was a seven factor solution, including 1) Impulsivity \& Externalizing, 2) Executive Functioning, 3) Internalizing, 4) Perceptual Reasoning, 5) Adaptability, 6) Processing Speed, and 7) Verbal Intelligence. Items from the parent-report measures of behavior and executive functioning tended to load together on factors, while items from the examiner-measured scales of intelligence loaded separately from the behavior and EF measures. The factors also generally aligned with the composite indices from the instruments.

To assess the utility of using factor scores over instrument-specific composite scores, we compared previously published well-characterized associations between smoking and neurodevelopment against estimates in our data using 1) instrument-specific composite scores, and 2) orthogonal varimax rotated factor scores. In prior literature, smoking has been consistently associated with impulsivity and externalizing behaviors, internalizing behaviors, and deficits in executive functioning, and less consistently associated with adaptive behaviors (Brion 
et al., 2010; Daseking et al., 2015; Indredavik et al., 2007; Piper \& Corbett, 2012; Stene-Larsen, Borge, \& Vollrath, 2009; Williams et al., 1998). With the exception of internalizing behaviors, we replicated this data in our population with the instrument-specific composite scores. However, after adopting the orthogonal varimax rotation, which imposes orthogonality on the factors, only the association between smoking and Impulsivity/Externalizing remained. This suggests that the association between smoking and the other characteristics may, at least in part, be driven by associations between smoking and impulsivity/externalizing. We also reported associations between canned fish consumption, maternal education, preterm birth, and home environmental organization scores and neurodevelopment, all of which were consistent with the literature.

We also assessed associations between prenatal exposure to OPs and neurodevelopmental factor scores and the Working Memory Index, while controlling for the coexposures of phthalates, phenols, and pyrethroid pesticides in a Bayesian framework. We reported associations between $\Sigma$ DMPs and worse Internalizing scores, but better Executive Functioning scores. We also reported associations between $\sum$ DEPs and worse performance on the Working Memory Index. There is some prior support for the associations between OPs and internalizing behaviors and working memory (Beard et al., 2014; V. Rauh et al., 2011). However, prior research on associations between prenatal exposure to $\Sigma$ DMPs and parent-reported executive functioning is limited to one other study that only used parent reports of attention or ADHD behaviors, and not other markers of executive functioning. In that study, ¿DMPs were associated with worse attention and parent-reported ADHD (Marks et al., 2010), which conflicts with our findings. However, the $\Sigma$ DMP pesticide source in the Marks population, which was primarily agricultural workers, is likely different than the $\Sigma$ DMP pesticide source in our population, which is composed of urban city-dwellers. Thus the exposures might not be comparable across the two studies. This possibility is one unfortunate drawback to working with the DAP biomarkers (discussed in more detail below). 
Finally, we initially hypothesized that OPs might be associated with ADHD, and that factor analysis might reveal an ADHD phenotype. However, no single factor seemed to capture the range of behaviors observed in ADHD. Rather, in our general population sample, we derived several factors that are implicated in ADHD, including Impulsivity and Externalizing, and Executive Functioning. OPs did not appear associated with Impulsivity and Externalizing, and DMPs were actually positively associated with the Executive Functioning factor, two hallmark deficits of ADHD, while DEPs were associated with worse Working Memory Index scores. As discussed previously, residual confounding may explain some of the association with executive functioning. Regardless, we did not observe the hypothesized association between OPs and an ADHD phenotype.

\section{Strengths and Limitations}

\section{Strengths}

There were several strengths to this dissertation. We used a longitudinal, multiethnic cohort that included both mostly white mothers from a wealthy neighborhood in Manhattan, as well as mostly minority mothers from the low-income neighborhood of East Harlem. Although the two groups display widely divergent socioeconomic features and may have different sources of parent pesticide exposure for their OP metabolite measurements, there was minimal interaction by race in both studies, and the factor structure was consistent by race. Thus, our findings generally apply across the scope of socioeconomic strata represented in an urban population of New York City.

We were able to adopt a novel framework for analyzing etiological associations with neurodevelopmental phenotypes that accounted for the complex correlational structure of neurobehavior. Dimension reduction enabled simultaneous examination of a wide range of neurobehavioral outcomes in an easily comparable format that collapsed over thirty separate outcomes into seven distinct factors. This method also allowed the assessment of etiological 
associations with individual neurodevelopmental phenotypes that were independent of associations with other neurodevelopmental phenotypes. Phenotyping may also capture information over and above information captured in instrument-specific composite scores alone. Estimates of associations between OPs and factor scores appeared to be stronger and slightly more precise than the estimates between OPs and most of the individual items that loaded highly on the relevant factor. Additionally, as the factor loadings of items decreased, the associations between OPs and the items attenuated to the null. This highlights another advantage of using factor analysis; if we were to examine the individual items from an instrument, OPs might appear associated with some items but not others in a somewhat haphazard fashion. Performing a factor analysis, however, allows the user to order items according to the strength of their factor loading, which implements an interpretable structure on the items.

Finally, we incorporated a mixture modeling approach to assess associations between OPs and neurodevelopment in a Bayesian framework, which stabilizes estimates from models with high numbers of variables and high collinearity among the covariates.

\section{Limitations}

Primary limitations include loss to follow-up, lack of corroborating data on parent pesticide exposure, use of a spot-urine sample in pregnancy to assess DAPs, unmeasured covariates, reporting bias, and lack of multi-method measurements of behavior and executive functioning.

Approximately $60 \%$ of the sample from the original cohort did not return for at least one complete neurodevelopmental follow-up visit between the ages of 6-9. This is worrisome for two primary reasons: 1) this resulted in smaller sample sizes, and 2) this loss-to-follow up presents a possibility for bias. Although the limited sample size did not prevent us from generating a reliable factor structure or from observing associations between smoking or OPs and 
neurodevelopment, there may have been repercussions for estimating interaction effects. In Chapter V, we only reported one interaction, between race and DMPs on Working Memory. Other interactions, particularly with PON1 or child sex, may have become apparent with a larger sample size and more participants per strata. Finally, the loss to follow-up may have resulted in biased estimates, if women who returned for follow-up were different than those who did not. Although the only measured characteristic that differed between the two groups was marital status, other unmeasured characteristics may confound relationships. This may have played a role particularly in estimating the relationship between DMPs and executive functioning: mothers with stronger executive functioning capacity may be more likely to eat a diet rich in fruits and vegetables during pregnancy (and thus have higher OP biomarkers while experiencing less toxicity), have children with better executive functioning, and be more likely to return for followup. Such a concern might be alleviated by controlling for maternal measures of executive functioning, or improved dietary exposure ascertainment methods; however these assessments were unfortunately not collected in this study.

Other measures of maternal psychiatric functioning or personality might have been important for other estimates. Controlling for maternal anxiety might increase precision of the DMP-Internalizing association, since maternal anxiety during pregnancy is associated with childhood anxiety (Van den Bergh, Mulder, Mennes, \& Glover, 2005). Additionally, controlling for personality, addictive tendencies, and general psychiatric functioning of the mother might address concerns about possible confounding of the smoking-impulsivity/externalizing relationship, since women who are unable to quit smoking during pregnancy might struggle with higher levels of impulsivity or addiction than women who are able to quit, and such traits might be heritable in nature. Again, these measures were not collected in this study.

Another limitation of this study is the reliance on self-reported cigarette use to assess smoking during pregnancy. Alternate measures, such as cotinine levels throughout pregnancy, may have reduced exposure misclassification by identifying mothers who smoked but did not 
report smoking during pregnancy. We also did not include information on environmental tobacco smoke exposure during pregnancy or childhood. While most studies report on smoking during the prenatal window of exposure, children of mothers who smoke during pregnancy are most likely also exposed to tobacco smoke throughout infancy and childhood. The brain grows rapidly and undergoes dramatic neural differentiation and pruning during the first two years of life, thus the idea that early-life programming may take place after pregnancy is highly plausible.

OP measurements during early childhood might have similarly provided interesting results. One cohort has observed associations between OP exposure during early childhood and neurodevelopment (Marks et al., 2010), but there is little supporting evidence for this in other cohorts due to the lack of biomarkers collected during childhood. Additionally, OP measurements throughout pregnancy could have helped describe a possible window of susceptibility, and possibly an effect for duration of exposure. Since the half-lives of most OPs range from hours to days, these measurements only reflect very recent exposure. Correlation coefficients of repeat measures of OPs in pregnancy range from 0.23 to 0.56 , while the intraclass correlation coefficient of OPs is approximately 0.40 (Egeghy et al., 2011; Whyatt et al., 2009). While there are competing hypotheses regarding the "most susceptible" window of exposure, it is perhaps equally plausible that exposures at different time periods have different effects, since the brain is continually developing throughout pregnancy. Alternatively, consistent exposure throughout pregnancy might be substantially worse than a single high exposure during any given trimester. Unfortunately, the results from using a single spot urine sample in the third trimester preclude assessing any of these possibilities.

Use of DAP biomarkers in general have other limitations. Up to $86 \%$ of samples on fruits and vegetables may be pre-formed metabolites rather than parent compounds (Zhang et al., 2008). Thus, exposure to direct parent compounds and exposure to preformed metabolites confer different toxicity levels that are not represented by DAP biomarkers. Although these concerns might be somewhat alleviated by measurements of maternal dietary intake during 
pregnancy, such information would still not shed light on the amount of toxic oxons a participant was exposed to via diet. This information would only come from sampling the actual produce that was consumed. Another limitation of DAP biomarkers is the non-specificity of the metabolites - six OP metabolites represent $\sim 30$ registered pesticides (Wessels et al., 2003). These parent pesticides vary in their toxicities, and DAP biomarkers may represent different pesticides for different populations. These biomarkers may thus be more useful for populations experiencing a consistent exposure to a single OP pesticide, or to multiple OP pesticides with similar toxicities. Single spot urines of DAP biomarkers also do not capture differences in individual excretion patterns or DAP metabolism, which may be important variables in estimating the individual toxicity of OP exposures. Despite these limitations, DAP biomarkers remain one of the preferred measurements of OP exposure due to their high detection frequencies relative to OP pesticide-specific biomarkers, which typically have low detection rates.

Finally, our study is somewhat limited by the lack of performance-based measurements of executive functioning in Aim 1. Although parent-report measures of executive functioning are not correlated with performance-based measures in either our population or in several previous studies, it is unclear what the disparity between the two measurements represents. Researchers have speculated that the two methods measure different dimensions of neurobehavior, or at least different dimensions of executive functioning. We did incorporate the performance-based measure of the Working Memory Index in our second aim, and found somewhat comparable associations between DMPs and the Executive Functioning factor and between DMPs and the Working Memory Index, although the latter was relatively imprecise. However, we also found associations between DEPs and worse scores on the Working Memory Index, but not on the Executive Functioning factor. These results are in seeming conflict in multiple ways; first, that DMPs and DEPs seem to have an opposite effect on executive functioning, and second, that DMPs were at least suggestively associated with two measures of executive functioning while 
DEPs were associated with just one. This conflicting pattern of associations might be easier to interpret if the meaning of the two measurements were clearer, and if more precise information was available regarding parent pesticide source and fruit and vegetable intake.

\section{Public Health Significance}

Organophosphorus pesticides have been banned from most residential uses (excluding roach bait stations and fire ant mound treatments) since 2001 , although they are still used widely in agriculture. Earlier this year (2016), the EPA proposed to revoke all food residue tolerances for the OP insecticide chlorpyrifos, with an anticipated release of the final rule in December of 2016. This would essentially result in the elimination of exposure to chlorpyrifos (which devolves into DEPs) for the vast majority of the American public. However, this ruling would not ban chlorpyrifos from all use; golf courses, turf, green houses, and non-structural wood treatments such as utility poles and fence posts, would not be affected by the proposed rule. The findings of this dissertation are timely, as we report an association between prenatal exposure to DEPs, of which chlorpyrifos is one parent pesticide, and Working Memory deficits. Although we cannot definitively identify the parent pesticide of DEPs in our cohort as chlorpyrifos, this result does support findings from a different birth cohort that reported associations between a specific chlorpyrifos metabolite and deficits on the Working Memory Index. However, we also report associations that show a relationship between DMPs and more adverse internalizing behaviors, particularly anxiety. Such research may contribute to policy decisions regarding OPs other than just chlorpyrifos.

Our findings of other modifiable characteristics that contribute to neurodevelopment may also be useful for advocating for healthy pregnancies. If the reported associations are causal, promoting higher education, encouraging mothers to eat low-mercury fish multiple times a week, preventing preterm birth, teaching mothers how to increase the predictability and organization of a child's environment, and offering assistance to quit smoking during pregnancy may have 
reverberating consequences on multiple dimensions of neurobehavior throughout childhood and possibly throughout life. The magnitude of the smoking and impulsivity and externalizing association was the highest of any of the modifiable risk factors and may result in the greatest gains, although this characteristic may simultaneously be the hardest to change due to the addictive nature of tobacco, and be the most subject to possible confounding by maternal traits. Of course, if the reported association between smoking and neurobehavior suffers from confounding by maternal traits, the magnitude of possible benefits will shrink. However, randomized trials of smoking cessation programs during pregnancy have resulted in both increasing the number of women who quit smoking relative to a control group, and reductions in preterm births and infants born with low birthweight (Chamberlain et al., 2013). These results already support the deployment of smoking cessation programs for pregnant women, and our results add another dimension to this argument.

Nationwide, the economic costs of neurodevelopmental deficits have been quantified, particularly in regards to early childhood lead exposure. Even small deficits, such as the loss of 0.67 IQ points on a population scale, translates to over $\$ 40$ billion lost in economic productivity over the course of a generation (Landrigan, Schechter, Lipton, Fahs, \& Schwartz, 2002). 0.67 IQ points is roughly 0.05 standard deviations. Several of our estimated beta coefficients, which can be interpreted as changes in standard deviations, were substantially larger than this for the IQ factors. While the economic costs of behavioral problems have yet to be quantified in such a manner, anxiety disorders were estimated to cost the US more than $\$ 42$ billion per year in the 1990s (Greenberg et al., 1999). Even a small 10\% reduction in anxiety disorders would translate to savings of $\$ 4$ billion. Thus, preventing these costs by addressing modifiable environmental exposures, behaviors, and parenting techniques could have compound public health and economic benefits. 


\section{Directions for Future Research}

Future research of prenatal and early life determinants of childhood neurodevelopment would ideally incorporate parent/self, and teacher-reports of behavior and executive functioning, as well as performance-based measures of executive functioning. Such approaches may help to elucidate differences attributed to measurement method and reporter.

Additionally, future research of OPs may be better served by incorporating nonbiomarker measurements of insecticide use, such as personal reports of fruit and vegetable consumption, personal pesticide use and pesticide exposure, or external reports such as state geographical records of pesticide applications. This information might enhance the accuracy of DAP biomarkers as a measurement of OP exposure.

Some of the best support for findings in epidemiologic research involves the replication of findings in other populations. Only three U.S. birth cohorts have reported associations between prenatal exposure to OPs and adverse childhood outcomes. However, creating cohorts and measuring biomarkers is an expensive endeavor, particularly during an era of limited $\mathrm{NIH}$ funding. Alternative approaches to assessing prenatal exposure to pesticides and childhood neurodevelopment might be more fruitful; for example, linking pesticide use data with childhood academic achievement or medical records might be one inexpensive way of assessing at least some of these associations in other populations.

Finally, findings of associations between prenatal exposure to OPs and neurological outcomes has thus far been limited to childhood neurodevelopment. Assessing associations with outcomes throughout the life-course might provide a better assessment of the true burden that OPs place on society. 


\section{Conclusions}

We reported several associations between prenatal and early life characteristics and neurodevelopment. Modifiable characteristics included maternal smoking during pregnancy, maternal canned fish consumption during pregnancy, organization of the home environment, and maternal education, while affected neurodevelopmental domains included measures of behavior, executive functioning, and IQ. We also reported associations between DMPs and worse Internalizing factor scores, between DMPs and better Executive Functioning factor scores, and between DEPs and more adverse Working Memory Index scores, after accounting for the complex correlational structure of several neurodevelopmental outcomes. 


\section{APPENDIX 1 DSM V CRITERIA FOR ADHD}

Three types of ADHD may occur: If enough symptoms are present in both Inattention and HyperactivityImpulsivity, this is Combined Presentation. If enough symptoms are present in Inattention but not Hyperactivity-Impulsivity, this is Predominantly Inattentive. If enough symptoms are present in Hyperactivity-Impulsivity, but not Inattention, this is Predominantly Hyperactive-Impulsive.

Inattention

Six or more symptoms of inattention for children up to age 16, five or more for adolescents 17 and older and adults, symptoms of inattention have been present for at least 6 months, and are inappropriate for developmental level

- Often fails to give close attention to details or makes careless mistakes in schoolwork, at work, or with other activities.

- Often has trouble holding attention on tasks or play activities.

- Often does not seem to listen when spoken to directly.

- Often does not follow through on instructions and fails to finish schoolwork, chores, or duties in the workplace (e.g., loses focus, side-tracked).

- Often has trouble organizing tasks and activities.

- Often avoids, dislikes, or is reluctant to do tasks that require mental effort over a long period of time (such as schoolwork or homework).

- Often loses things necessary for tasks and activities (e.g. school materials, pencils, books, tools, wallets, keys, paperwork, eyeglasses, mobile telephones).

- Is often easily distracted

- Is often forgetful in daily activities.

Hyperactivity and Impulsivity:

Six or more symptoms of hyperactivity-impulsivity for children up to age 16, or five or more for adolescents 17 and older and adults; symptoms of hyperactivity-impulsivity have been present for at least 6 months to an extent that is disruptive and inappropriate for the person's developmental level:

- Often fidgets with or taps hands or feet, or squirms in seat.

- Often leaves seat in situations when remaining seated is expected.

- Often runs about or climbs in situations where it is not appropriate (adolescents or adults may be limited to feeling restless).

- Often unable to play or take part in leisure activities quietly.

- Is often "on the go" acting as if "driven by a motor".

- Often talks excessively.

- Often blurts out an answer before a question has been completed.

- Often has trouble waiting his/her turn.

- Often interrupts or intrudes on others (e.g., butts into conversations or games)

In addition, the following conditions must be met

- Several inattentive or hyperactive-impulsive symptoms were present before age 12 years.

- Several symptoms are present in two or more setting, (e.g., at home, school or work; with friends or relatives; in other activities).

- There is clear evidence that the symptoms interfere with, or reduce the quality of, social, school, or work functioning.

- The symptoms do not happen only during the course of schizophrenia or another psychotic disorder. The symptoms are not better explained by another mental disorder (e.g. Mood Disorder, Anxiety Disorder, Dissociative Disorder, or a Personality Disorder). 


\section{APPENDIX 2 PROMAX ROTATED FACTOR PATTERN STRUCTURE AND ITEM LOADINGS OF CHILDHOOD NEURODEVELOPMENTAL SCALES ( $\mathrm{N}=\mathbf{2 1 0})$}

\begin{tabular}{|c|c|c|c|c|c|c|c|}
\hline \multicolumn{2}{|c|}{$\begin{array}{c}\text { Factor } 1 \\
\text { Impulsivity \& } \\
\text { Externalizing }\end{array}$} & \multicolumn{2}{|c|}{$\begin{array}{c}\text { Factor } 2 \\
\text { Executive Functioning }\end{array}$} & \multicolumn{2}{|c|}{$\begin{array}{c}\text { Factor } 3 \\
\text { Internalizing }\end{array}$} & \multicolumn{2}{|c|}{$\begin{array}{c}\text { Factor } 4 \\
\text { Perceptual Reasoning }\end{array}$} \\
\hline Scale & Loading & Scale & Loading & Scale & Loading & $\begin{array}{l}\text { Scale } \\
\text { Perceptual }\end{array}$ & Loading \\
\hline Externalizing & & Metacognition & & Internalizing & & Reasoning & \\
\hline Problems & 0.97 & Index & 0.96 & Problems & 0.89 & $\begin{array}{l}\text { IQ } \\
\text { Picture }\end{array}$ & 0.98 \\
\hline Conduct & 0.89 & $\begin{array}{l}\text { Planning } \\
\text { Working }\end{array}$ & 0.94 & Anxiety & 0.81 & $\begin{array}{l}\text { Concepts } \\
\text { Matrix }\end{array}$ & 0.78 \\
\hline Aggression & 0.85 & Memory & 0.81 & Withdrawal & 0.77 & $\begin{array}{l}\text { Reasoning } \\
\text { Block }\end{array}$ & 0.74 \\
\hline Hyperactivity & 0.75 & Initiate & 0.81 & Somatization & 0.69 & Design & 0.71 \\
\hline $\begin{array}{l}\text { Inhibit } \\
\text { Behavioral } \\
\text { Regulation }\end{array}$ & 0.62 & $\begin{array}{l}\text { Monitor } \\
\text { Global } \\
\text { Executive }\end{array}$ & 0.80 & Depression & 0.59 & Full Scale IQ & 0.50 \\
\hline Index & 0.61 & Composite & 0.75 & $\begin{array}{l}\text { Atypicality } \\
\text { Behavioral } \\
\text { Symptoms }\end{array}$ & 0.54 & & \\
\hline $\begin{array}{l}\text { Adaptability } \\
\text { Behavioral } \\
\text { Symptoms }\end{array}$ & -0.59 & Organization & 0.66 & Index & 0.40 & & \\
\hline $\begin{array}{l}\text { Index } \\
\text { Emotional }\end{array}$ & 0.58 & Attention & 0.49 & $\begin{array}{l}\text { Shift } \\
\text { Emotional }\end{array}$ & 0.38 & & \\
\hline Control & 0.57 & $\begin{array}{l}\text { Inhibit } \\
\text { Behavioral } \\
\text { Regulation }\end{array}$ & 0.46 & Control & 0.31 & & \\
\hline Shift & 0.40 & Index & 0.32 & & & & \\
\hline $\begin{array}{l}\text { Depression } \\
\text { Attention }\end{array}$ & $\begin{array}{l}0.34 \\
0.32\end{array}$ & & & & & & \\
\hline Attention & 0.32 & & & & & & \\
\hline $\begin{array}{l}\text { Factor } \\
\text { Structure } \\
\% \text { Variance } \\
\text { accounted for }\end{array}$ & 37.92 & & 13.71 & & 7.86 & & 6.33 \\
\hline Eigenvalue & 14.03 & & 5.07 & & 2.91 & & 2.34 \\
\hline
\end{tabular}

${ }^{1}$ BASC scales, ${ }^{2}$ BRIEF scales, ${ }^{3}$ WISC-IV or the WPPSI-III scales. Only items with loadings with absolute values $>0.30$ are shown here. Loadings are from a PCA with oblique promax rotation (factors are allowed to correlate) 
continued

\begin{tabular}{|c|c|c|c|c|c|}
\hline \multicolumn{2}{|c|}{$\begin{array}{c}\text { Factor } 5 \\
\text { Adaptability }\end{array}$} & \multicolumn{2}{|c|}{$\begin{array}{c}\text { Factor } 6 \\
\text { Processing Speed }\end{array}$} & \multicolumn{2}{|c|}{$\begin{array}{c}\text { Factor } 7 \\
\text { Verbal Intelligence }\end{array}$} \\
\hline Scale & Loading & $\begin{array}{l}\text { Scale } \\
\text { Processing } \\
\text { Speed }\end{array}$ & Loading & Scale & Loading \\
\hline Skills Index & 0.89 & Index ${ }^{3}$ & 0.96 & Vocabulary $^{3}$ & 0.85 \\
\hline Leadership & 0.85 & $\begin{array}{l}\text { Coding }^{3} \\
\text { Symbol }\end{array}$ & 0.88 & $\begin{array}{l}\text { Verbal } I^{3} \\
\text { Full Scale }\end{array}$ & 0.83 \\
\hline Social Skills & 0.85 & $\begin{array}{l}\text { Search } \\
\text { Full Scale }\end{array}$ & 0.71 & $\begin{array}{l}1 \mathrm{O}^{3} \\
\text { Organization }\end{array}$ & 0.57 \\
\hline Adaptability & 0.56 & $1 Q^{3}$ & 0.36 & $\begin{array}{l}\text { Symbol } \\
\text { Search }^{3}\end{array}$ & 0.38 \\
\hline & 5.10 & & 4.25 & & 3.05 \\
\hline & 1.89 & & 1.57 & & 1.13 \\
\hline
\end{tabular}




\section{APPENDIX 3 ASSOCIATIONS BETWEEN SMOKING AND NEURODEVELOPMENTAL OUTCOMES, BY OUTCOME MEASUREMENT METHOD}

Comparison of Prenatal Smoking and Neurodevelopment Associations by Analysis Method ( $n=162)$

\begin{tabular}{|c|c|c|c|}
\hline & $\begin{array}{l}\text { Any smoking during } \\
\text { pregnancy }\end{array}$ & $\begin{array}{l}\text { Smoking in First } \\
\text { Trimester Only }\end{array}$ & $\begin{array}{l}\text { Smoking in Second or } \\
\text { Third Trimesters }\end{array}$ \\
\hline Impulsivity \& Externalizing factor, & & & \\
\hline $\begin{array}{l}\text { Varimax } \\
\text { Impulsivity \& Externalizing factor, }\end{array}$ & $-0.51(-0.92,-0.10)$ & $-0.29(-0.80,0.22)$ & $-0.82(-1.42,-0.23)$ \\
\hline Promax $^{1}$ & $-0.51(-0.92,-0.10)$ & $-0.27(-0.79,0.24)$ & $-0.84(-1.45,-0.24)$ \\
\hline Externalizing Composite $^{3}$ & $-0.60(-1.00,-0.21)$ & $-0.36(-0.84,0.13)$ & $-0.97(-1.55,-0.39)$ \\
\hline Executive Functioning factor, & & & \\
\hline Varimax ${ }^{2}$ & $-0.32(-0.75,0.10)$ & $-0.32(-0.85,0.21)$ & $-0.32(-0.94,0.30)$ \\
\hline Executive Functioning factor, & & & \\
\hline Promax & $-0.43(-0.85,-0.02)$ & $-0.30(-0.82,0.22)$ & $-0.63(-1.24,-0.02)$ \\
\hline Metacognition Index ${ }^{3}$ & $-0.47(-0.86,-0.09)$ & $-0.23(-0.71,0.25)$ & $-0.55(-1.12,0.03)$ \\
\hline Internalizing factor, Varimax ${ }^{2}$ & $0.26(-0.16,0.68)$ & $0.32(-0.21,0.84)$ & $0.18(-0.43,0.80)$ \\
\hline Internalizing factor, Promax ${ }^{1}$ & $-0.03(-0.45,0.39)$ & $0.11(-0.41,0.63)$ & $-0.24(-0.85,0.38)$ \\
\hline Internalizing Composite ${ }^{3}$ & $0.07(-0.33,0.47)$ & $0.18(-0.32,0.68)$ & $-0.09(-0.68,0.50)$ \\
\hline $\begin{array}{l}\text { Perceptual Reasoning factor, } \\
\text { Varimax }\end{array}$ & $0.06(-0.33,0.45)$ & $0.28(-0.21,0.77)$ & $-0.26(-0.83,0.31)$ \\
\hline Perceptual Reasoning factor, & & & \\
\hline Promax $^{1}$ & $0.06(-0.31,0.42)$ & $0.25(-0.21,0.71)$ & $-0.22(-0.76,0.31)$ \\
\hline Perceptual Reasoning $1 \mathrm{Q}^{3}$ & $0.14(-0.25,0.53)$ & $0.37(-0.11,0.85)$ & $-0.19(-0.76,0.38)$ \\
\hline Adaptability factor, Varimax ${ }^{2}$ & $-0.08(-0.48,0.33)$ & $0.06(-0.45,0.56)$ & $-0.27(-0.85,0.32)$ \\
\hline Adaptability factor, Promax ${ }^{1}$ & $-0.27(-0.67,0.14)$ & $-0.10(-0.60,0.41)$ & $-0.51(-1.10,0.08)$ \\
\hline Adaptive Skills Composite 3 & $-0.33(-0.71,0.06)$ & $-0.10(-0.58,0.37)$ & $-0.66(-1.23,-0.10)$ \\
\hline Processing Speed factor, Varimax ${ }^{2}$ & $-0.16(-0.57,0.26)$ & $-0.09(-0.61,0.43)$ & $-0.26(-0.87,0.36)$ \\
\hline Processing Speed factor, Promax ${ }^{1}$ & $-0.19(-0.60,0.23)$ & $-0.10(-0.62,0.42)$ & $-0.32(-0.93,0.29)$ \\
\hline Processing Speed $1 Q^{3}$ & $-0.22(-0.66,0.23)$ & $-0.11(-0.69,0.48)$ & $-0.46(-1.07,0.15)$ \\
\hline Verbal Intelligence, Varimax ${ }^{2}$ & $0.04(-0.31,0.40)$ & $-0.12(-0.56,0.32)$ & $0.28(-0.23,0.79)$ \\
\hline Verbal Intelligence, Promax ${ }^{1}$ & $0.11(-0.21,0.42)$ & $0.07(-0.32,0.46)$ & $0.17(-0.29,0.63)$ \\
\hline Verbal $1 Q^{3}$ & $0.15(-0.16,0.46)$ & $0.10(-0.29,0.49)$ & $0.22(-0.24,0.68)$ \\
\hline
\end{tabular}

All models adjusted for maternal race, maternal education at follow-up, alcohol consumption during pregnancy, and HOME scores

${ }^{1}$ Promax (oblique) rotation allows factors to correlate

${ }^{2}$ Varimax (orthogonal) rotation factors are statistically uncorrelated with each other.

${ }^{3}$ The composite/index items are the highest loading composite item for each factor. 


\section{APPENDIX 4 BIVARIATE ASSOCIATIONS BETWEEN EARLY LIFE CHARACTERISTICS AND NEURODEVELOPMENTAL FACTORS ${ }^{1}$ IN THE MOUNT SINAI CHILDREN'S ENVIRONMENTAL HEALTH CENTER}

\begin{tabular}{|c|c|c|c|c|c|c|c|c|}
\hline & $\mathrm{N}$ & $\begin{array}{c}\text { Factor } 1 \\
\text { Impulsivity } \\
\text { \&Externalizing } \\
\beta(95 \% \mathrm{Cl})\end{array}$ & $\begin{array}{c}\text { Factor } 2 \\
\text { Executive } \\
\text { Functioning } \\
\beta(95 \% \mathrm{Cl})\end{array}$ & $\begin{array}{c}\text { Factor } 3 \\
\text { Internalizing } \\
\beta(95 \% \mathrm{Cl})\end{array}$ & $\begin{array}{c}\text { Factor } 4 \\
\text { Perceptual } \\
\text { Reasoning } \\
\beta(95 \% \mathrm{Cl})\end{array}$ & $\begin{array}{c}\text { Factor } 5 \\
\text { Adaptability } \\
\beta(95 \% \mathrm{Cl})\end{array}$ & $\begin{array}{c}\text { Factor } 6 \\
\text { Processing } \\
\quad \text { Speed } \\
\beta(95 \% \mathrm{Cl})\end{array}$ & $\begin{array}{c}\text { Factor } 7 \\
\text { Verbal } \\
\text { Intelligence } \\
\beta(95 \% \mathrm{Cl})\end{array}$ \\
\hline \multicolumn{9}{|c|}{ Mothers Marital Status at Follow up } \\
\hline Married & 61 & referent & referent & referent & referent & referent & referent & referent \\
\hline $\begin{array}{l}\text { Living with } \\
\text { Partner }\end{array}$ & 39 & $\begin{array}{l}0.12(-0.28 \\
0.51)\end{array}$ & $\begin{array}{l}0.20(-0.20 \\
0.61)\end{array}$ & $\begin{array}{l}0.11(-0.30 \\
0.51)\end{array}$ & $\begin{array}{l}-0.29(-0.69 \\
0.10)\end{array}$ & $\begin{array}{l}-0.25(-0.64 \\
0.14)\end{array}$ & $\begin{array}{l}0.20(-0.20 \\
0.59)\end{array}$ & $\begin{array}{l}-0.48(-0.87,- \\
0.08)\end{array}$ \\
\hline $\begin{array}{l}\text { Single/Divorced/ } \\
\text { Widowed }\end{array}$ & & $\begin{array}{l}-0.27(-0.58 \\
0.05)\end{array}$ & $\begin{array}{l}0.07(-0.25 \\
0.39)\end{array}$ & $\begin{array}{l}-0.02(-0.34 \\
0.30)\end{array}$ & $\begin{array}{l}-0.41(-0.73,- \\
0.10)\end{array}$ & $\begin{array}{l}-0.46(-0.76,- \\
0.15)\end{array}$ & $\begin{array}{l}0.09(-0.23 \\
0.40)\end{array}$ & $\begin{array}{l}-0.33(-0.65,- \\
0.02)\end{array}$ \\
\hline Pr>chisq & & 0.07 & 0.61 & 0.80 & 0.04 & 0.02 & 0.62 & 0.04 \\
\hline \multicolumn{9}{|l|}{ Maternal IQ } \\
\hline $\mathrm{IQ}<100$ & 91 & referent & referent & referent & referent & referent & referent & referent \\
\hline$I Q \geq 100$ & 45 & $\begin{array}{l}0.12(-0.24 \\
0.48)\end{array}$ & $\begin{array}{l}-0.49(-0.85,- \\
0.13)\end{array}$ & $\begin{array}{l}-0.04(-0.38 \\
0.30)\end{array}$ & $\begin{array}{l}0.54(0.19 \\
0.89)\end{array}$ & $\begin{array}{l}0.18(-0.18 \\
0.53)\end{array}$ & $\begin{array}{l}0.06(-0.28 \\
0.39)\end{array}$ & $\begin{array}{l}0.80(0.51 \\
1.10)\end{array}$ \\
\hline Pr> chisq & & 0.50 & 0.01 & 0.82 & $<0.01$ & 0.32 & 0.74 & $<0.01$ \\
\hline \multicolumn{9}{|c|}{ Maternal Education at Follow Up } \\
\hline $\begin{array}{l}\text { High school or } \\
\text { less }\end{array}$ & 84 & referent & referent & referent & referent & referent & referent & referent \\
\hline Some college & 81 & $\begin{array}{l}-0.06(-0.36 \\
0.24)\end{array}$ & $\begin{array}{l}-0.26(-0.56 \\
0.04)\end{array}$ & $\begin{array}{l}0.15(-0.16 \\
0.45)\end{array}$ & $\begin{array}{l}0.04(-0.25 \\
0.33)\end{array}$ & $\begin{array}{l}0.07(-0.23 \\
0.37)\end{array}$ & $\begin{array}{l}-0.15(-0.46 \\
0.15)\end{array}$ & $\begin{array}{l}0.55(0.28 \\
0.82)\end{array}$ \\
\hline $\begin{array}{l}\text { Bachelor's } \\
\text { degree }\end{array}$ & 45 & $\begin{array}{l}-0.16(-0.52 \\
0.21)\end{array}$ & $\begin{array}{l}-0.33(-0.69 \\
0.03)\end{array}$ & $\begin{array}{l}0.18(-0.18 \\
0.54)\end{array}$ & $\begin{array}{l}0.75(0.40 \\
1.09)\end{array}$ & $\begin{array}{l}0.46(0.11, \\
0.82)\end{array}$ & $\begin{array}{l}0.05(-0.31 \\
0.41)\end{array}$ & $\begin{array}{l}1.18(0.85 \\
1.50)\end{array}$ \\
\hline Pr> chisq & & 0.70 & 0.11 & 0.51 & $<0.01$ & 0.03 & 0.47 & $<0.01$ \\
\hline \multicolumn{9}{|c|}{ Maternal Age at Delivery } \\
\hline$<20$ & 98 & referent & referent & referent & referent & referent & referent & referent \\
\hline $20-25$ & 55 & $\begin{array}{l}-0.11(-0.44, \\
0.22)\end{array}$ & $\begin{array}{l}0.01(-0.32 \\
0.34)\end{array}$ & $\begin{array}{l}0.13(-0.20 \\
0.46)\end{array}$ & $\begin{array}{l}0.09(-0.22 \\
0.40)\end{array}$ & $\begin{array}{l}0.06(-0.26 \\
0.38)\end{array}$ & $\begin{array}{l}-0.22(-054 \\
0.11)\end{array}$ & $\begin{array}{l}0.28(-0.04 \\
0.59)\end{array}$ \\
\hline$>25$ & 57 & $\begin{array}{l}-0.18(-0.50 \\
0.15)\end{array}$ & $\begin{array}{l}-0.31(-0.63 \\
0.02)\end{array}$ & $\begin{array}{l}-0.05(-0.38 \\
0.27)\end{array}$ & $\begin{array}{l}0.70(0.39 \\
1.01)\end{array}$ & $\begin{array}{l}0.50(0.18 \\
0.82)\end{array}$ & $\begin{array}{l}-0.16(-0.48 \\
0.17)\end{array}$ & $\begin{array}{l}0.74(0.43, \\
1.05)\end{array}$ \\
\hline Pr $>$ chisq & & 0.54 & 0.13 & 0.61 & $<0.01$ & $<0.01$ & 0.38 & $<0.01$ \\
\hline \multicolumn{9}{|l|}{ Maternal Race } \\
\hline $\begin{array}{l}\text { Black or other } \\
\text { race }\end{array}$ & 57 & referent & referent & referent & referent & referent & referent & referent \\
\hline White & 31 & $\begin{array}{l}0.01(-0.43 \\
0.44)\end{array}$ & $\begin{array}{l}-0.33(-0.76, \\
0.10)\end{array}$ & $\begin{array}{l}0.07(-0.37 \\
0.50)\end{array}$ & $\begin{array}{l}1.14(0.74, \\
1.55)\end{array}$ & $\begin{array}{l}0.30(-0.13 \\
0.74)\end{array}$ & $\begin{array}{l}-0.01(-0.45 \\
0.43)\end{array}$ & $\begin{array}{l}1.05(0.65, \\
1.44)\end{array}$ \\
\hline Hispanic & 122 & $\begin{array}{l}0.22(-0.09 \\
0.53)\end{array}$ & $\begin{array}{l}-0.28(-0.59 \\
0.03)\end{array}$ & $\begin{array}{l}-0.04(-0.35 \\
0.28)\end{array}$ & $\begin{array}{l}0.14(-0.15 \\
0.43)\end{array}$ & $\begin{array}{l}-0.00(-0.31 \\
0.31)\end{array}$ & $\begin{array}{l}-0.00(-0.31 \\
0.31)\end{array}$ & $\begin{array}{l}-0.25(-0.53 \\
0.03)\end{array}$ \\
\hline Pr> chisq & & 0.29 & 0.16 & 0.87 & $<0.01$ & 0.29 & 0.99 & $<0.01$ \\
\hline \multicolumn{9}{|c|}{ Maternal Smoking During Pregnancy } \\
\hline None & 134 & referent & referent & referent & referent & referent & referent & referent \\
\hline Any & 28 & $\begin{array}{l}-0.53(-0.91,- \\
0.15)\end{array}$ & $\begin{array}{l}-0.31(-0.73 \\
0.10)\end{array}$ & $\begin{array}{l}0.26(-0.14 \\
0.66)\end{array}$ & $\begin{array}{l}0.02(-0.38 \\
0.43)\end{array}$ & $\begin{array}{l}-0.18(-0.59 \\
0.23)\end{array}$ & $\begin{array}{l}-0.23(-0.62 \\
0.17)\end{array}$ & $\begin{array}{l}-0.06(-0.44 \\
0.33)\end{array}$ \\
\hline
\end{tabular}




\begin{tabular}{|c|c|c|c|c|c|c|c|c|}
\hline & $\mathrm{N}$ & $\begin{array}{c}\text { Factor } 1 \\
\text { Impulsivity } \\
\text { \&Externalizing } \\
\beta(95 \% \mathrm{Cl})\end{array}$ & $\begin{array}{c}\text { Factor } 2 \\
\text { Executive } \\
\text { Functioning } \\
\beta(95 \% \mathrm{Cl})\end{array}$ & $\begin{array}{c}\text { Factor } 3 \\
\text { Internalizing } \\
\beta(95 \% \mathrm{Cl})\end{array}$ & $\begin{array}{c}\text { Factor } 4 \\
\text { Perceptual } \\
\text { Reasoning } \\
\beta(95 \% \mathrm{Cl})\end{array}$ & $\begin{array}{c}\text { Factor } 5 \\
\text { Adaptability } \\
\beta(95 \% \mathrm{Cl})\end{array}$ & $\begin{array}{c}\text { Factor } 6 \\
\text { Processing } \\
\text { Speed } \\
\beta(95 \% \mathrm{Cl})\end{array}$ & $\begin{array}{c}\text { Factor } 7 \\
\text { Verbal } \\
\text { Intelligence } \\
\beta(95 \% \mathrm{Cl})\end{array}$ \\
\hline Pr> chisq & & 0.01 & 0.14 & 0.20 & 0.91 & 0.39 & 0.26 & 0.77 \\
\hline \multicolumn{9}{|c|}{$\begin{array}{l}\text { Maternal Alcohol Use During } \\
\text { Pregnancy }\end{array}$} \\
\hline None & 132 & referent & referent & referent & referent & referent & referent & referent \\
\hline Any & 29 & $\begin{array}{l}0.08(-0.32, \\
0.48)\end{array}$ & $\begin{array}{l}-0.07(-0.50 \\
0.35)\end{array}$ & $\begin{array}{l}0.11(-0.30 \\
0.52)\end{array}$ & $\begin{array}{l}0.68(0.28, \\
1.09)\end{array}$ & $\begin{array}{l}0.07(-0.35 \\
0.48)\end{array}$ & $\begin{array}{l}-0.01(-0.41 \\
0.39)\end{array}$ & $\begin{array}{l}0.74(0.36, \\
1.12)\end{array}$ \\
\hline $\mathrm{Pr}>$ chisq & & 0.68 & 0.73 & 0.61 & $<0.01$ & 0.74 & 0.96 & $<0.01$ \\
\hline \multicolumn{9}{|c|}{$\begin{array}{l}\text { Maternal Canned Fish Consumption During } \\
\text { Pregnancy }\end{array}$} \\
\hline $\begin{array}{l}<1 \text { times per } \\
\text { week }\end{array}$ & 145 & referent & referent & referent & referent & referent & referent & referent \\
\hline $\begin{array}{l}1 \text { or more times } \\
\text { per week }\end{array}$ & 22 & $\begin{array}{l}-0.16(-0.59 \\
0.27)\end{array}$ & $\begin{array}{l}-0.27(-0.73 \\
0.18)\end{array}$ & $\begin{array}{l}0.24(-0.20 \\
0.68)\end{array}$ & $\begin{array}{l}0.83(0.40 \\
1.26)\end{array}$ & $\begin{array}{l}0.36(-0.09 \\
0.80)\end{array}$ & $\begin{array}{l}-0.17(-0.61 \\
0.27)\end{array}$ & $\begin{array}{l}0.32(-0.10 \\
0.74)\end{array}$ \\
\hline Pr>Chisq & & 0.47 & 0.24 & 0.29 & $<0.01$ & 0.12 & 0.45 & 0.14 \\
\hline \multicolumn{9}{|l|}{ Child Sex } \\
\hline Male & 100 & referent & referent & referent & referent & referent & referent & referent \\
\hline Female & 105 & $\begin{array}{l}0.35(0.08 \\
0.62)\end{array}$ & $\begin{array}{l}-0.11(-0.39 \\
0.16)\end{array}$ & $\begin{array}{l}-0.28(-0.55 \\
0.00)\end{array}$ & $\begin{array}{l}-0.27(-0.54 \\
0.00)\end{array}$ & $\begin{array}{l}0.42(0.15 \\
0.68)\end{array}$ & $\begin{array}{l}0.40(0.13 \\
0.67)\end{array}$ & $\begin{array}{l}-0.12(-0.39 \\
0.16)\end{array}$ \\
\hline Pr> chisq & & 0.01 & 0.41 & 0.05 & 0.05 & $<0.01$ & $<0.01$ & 0.40 \\
\hline \multicolumn{9}{|l|}{ Gestational Age } \\
\hline Preterm & 60 & $\begin{array}{l}0.28(-0.02 \\
0.57)\end{array}$ & $\begin{array}{l}-0.13(-0.43 \\
0.17)\end{array}$ & $\begin{array}{l}-0.34(-0.64,- \\
0.05)\end{array}$ & $\begin{array}{l}-0.19(-0.49 \\
0.10)\end{array}$ & $\begin{array}{l}-0.13(-0.43 \\
0.17)\end{array}$ & $\begin{array}{l}-0.15(-0.45 \\
0.14)\end{array}$ & $\begin{array}{l}-0.46(-0.75,- \\
0.16)\end{array}$ \\
\hline Pr> chisq & & 0.07 & 0.38 & 0.02 & 0.20 & 0.39 & 0.31 & $<0.01$ \\
\hline \multicolumn{9}{|c|}{ Head Circumference } \\
\hline $\begin{array}{l}\text { Centimeters, } \\
\text { continuous }\end{array}$ & 162 & $\begin{array}{l}-0.04(-0.14 \\
0.06)\end{array}$ & $\begin{array}{l}-0.12(-0.22,- \\
0.02)\end{array}$ & $\begin{array}{l}0.04(-0.06 \\
0.14)\end{array}$ & $\begin{array}{l}0.15(0.05 \\
0.24)\end{array}$ & $\begin{array}{l}0.00(-0.10 \\
0.10)\end{array}$ & $\begin{array}{l}0.07(-0.03 \\
0.17)\end{array}$ & $\begin{array}{l}0.06(-0.03 \\
0.16)\end{array}$ \\
\hline Pr> chisq & & 0.43 & 0.02 & 0.47 & $<0.01$ & 0.99 & 0.17 & 0.19 \\
\hline \multicolumn{9}{|l|}{ Birth weight } \\
\hline $\begin{array}{l}<\text { median }(< \\
3270 \mathrm{~g})\end{array}$ & 76 & referent & referent & referent & referent & referent & referent & referent \\
\hline $\begin{array}{l}\geq \text { Median ( } \geq \\
3270 \mathrm{~g})\end{array}$ & 86 & $\begin{array}{l}-0.25(-0.55 \\
0.04)\end{array}$ & $\begin{array}{l}-0.10(-0.41 \\
0.22)\end{array}$ & $\begin{array}{l}0.08(-0.23 \\
0.39)\end{array}$ & $\begin{array}{l}0.21(-0.09 \\
0.52)\end{array}$ & $\begin{array}{l}0.20(-0.11 \\
0.50)\end{array}$ & $\begin{array}{l}0.19(-0.11 \\
0.49)\end{array}$ & $\begin{array}{l}-0.12(-0.41 \\
0.17)\end{array}$ \\
\hline $\mathrm{Pr}>$ chisq & & 0.10 & 0.54 & 0.61 & 0.17 & 0.21 & 0.21 & 0.41 \\
\hline \multicolumn{9}{|l|}{ Birth Length } \\
\hline $\begin{array}{l}<\text { median }(<51 \\
\mathrm{cm})\end{array}$ & 75 & referent & referent & referent & referent & referent & referent & referent \\
\hline $\begin{array}{l}\geq \text { median }(\geq 51 \\
\mathrm{cm})\end{array}$ & 85 & $\begin{array}{l}-0.22(-0.52 \\
0.08)\end{array}$ & $\begin{array}{l}-0.17(-0.49 \\
0.14)\end{array}$ & $\begin{array}{l}0.33(0.02 \\
0.63)\end{array}$ & $\begin{array}{l}0.17(-0.14 \\
0.48)\end{array}$ & $\begin{array}{l}0.05(-0.27 \\
0.36)\end{array}$ & $\begin{array}{l}-0.02(-0.32 \\
0.29)\end{array}$ & $\begin{array}{l}-0.14(-0.44 \\
0.15)\end{array}$ \\
\hline Pr> chisq & & 0.15 & 0.29 & 0.03 & 0.29 & 0.77 & 0.90 & 0.34 \\
\hline
\end{tabular}




\begin{tabular}{|c|c|c|c|c|c|c|c|}
\hline & $\begin{array}{cc}N \quad \text { Factor } 1 \\
\text { Impulsivity } \\
\text { \&Externalizing } \\
\beta(95 \% \mathrm{Cl})\end{array}$ & $\begin{array}{c}\text { Factor } 2 \\
\text { Executive } \\
\text { Functioning } \\
\beta(95 \% \mathrm{Cl})\end{array}$ & $\begin{array}{c}\text { Factor } 3 \\
\text { Internalizing } \\
\beta(95 \% \mathrm{Cl})\end{array}$ & $\begin{array}{c}\text { Factor } 4 \\
\text { Perceptual } \\
\text { Reasoning } \\
\beta(95 \% \mathrm{Cl})\end{array}$ & $\begin{array}{c}\text { Factor } 5 \\
\text { Adaptability } \\
\beta(95 \% \mathrm{Cl})\end{array}$ & $\begin{array}{c}\text { Factor } 6 \\
\text { Processing } \\
\quad \text { Speed } \\
\beta(95 \% \mathrm{Cl})\end{array}$ & $\begin{array}{c}\text { Factor } 7 \\
\text { Verbal } \\
\text { Intelligence } \\
\beta(95 \% \mathrm{Cl})\end{array}$ \\
\hline \multicolumn{8}{|c|}{ HOME Observation for Measurement of the Environment Scores } \\
\hline $\begin{array}{l}\text { Overall Score } \\
\text { Continuous }\end{array}$ & $\begin{aligned} & 0.00(-0.02 \\
156 & 0.03)\end{aligned}$ & $\begin{array}{l}0.01(-0.01 \\
0.04)\end{array}$ & $\begin{array}{l}0.01(-0.02 \\
0.04)\end{array}$ & $\begin{array}{l}0.01(-0.02 \\
0.04)\end{array}$ & $\begin{array}{l}0.04(0.01 \\
0.06)\end{array}$ & $\begin{array}{l}0.02(0.00, \\
0.05)\end{array}$ & $\begin{array}{l}0.01(-0.01 \\
0.04)\end{array}$ \\
\hline Pr> chisq & 0.94 & 0.37 & 0.48 & 0.44 & $<0.01$ & 0.07 & 0.33 \\
\hline \multicolumn{8}{|l|}{ Responsivity } \\
\hline $\begin{array}{l}\text { Ordinal } \\
\text { categorical }\end{array}$ & $\begin{aligned} & 0.04(-0.14 \\
156 & 0.22)\end{aligned}$ & $\begin{array}{l}-0.01(-0.20 \\
0.18)\end{array}$ & $\begin{array}{l}0.12(-0.07 \\
0.30)\end{array}$ & $\begin{array}{l}0.05(-0.14 \\
0.23)\end{array}$ & $\begin{array}{l}0.13(-0.05 \\
0.31)\end{array}$ & $\begin{array}{l}0.01(-0.17 \\
0.19)\end{array}$ & $\begin{array}{l}0.15(-0.03 \\
0.32)\end{array}$ \\
\hline Pr> chisq & 0.64 & 0.89 & 0.21 & 0.63 & 0.15 & 0.88 & 0.09 \\
\hline \multicolumn{8}{|l|}{ Involvement } \\
\hline $\begin{array}{l}\text { Ordinal } \\
\text { categorical }\end{array}$ & $\begin{aligned} & 0.03(-0.16 \\
156 & 0.23)\end{aligned}$ & $\begin{array}{l}-0.04(-0.25 \\
0.16)\end{array}$ & $\begin{array}{l}0.04(-0.16 \\
0.24)\end{array}$ & $\begin{array}{l}0.22(0.02, \\
0.42)\end{array}$ & $\begin{array}{l}0.23(0.04 \\
0.42)\end{array}$ & $\begin{array}{l}0.09(-0.10 \\
0.29)\end{array}$ & $\begin{array}{l}0.13(-0.06, \\
0.32)\end{array}$ \\
\hline Pr> chisq & 0.76 & 0.68 & 0.70 & 0.03 & 0.02 & 0.36 & 0.19 \\
\hline \multicolumn{8}{|l|}{ Organization } \\
\hline $\begin{array}{l}\text { Ordinal } \\
\text { categorical }\end{array}$ & $\begin{array}{l}-0.11(-0.29 \\
1560.07)\end{array}$ & $\begin{array}{l}0.28(0.09, \\
0.47)\end{array}$ & $\begin{array}{l}0.21(0.02, \\
0.39)\end{array}$ & $\begin{array}{l}0.02(-0.17 \\
0.20)\end{array}$ & $\begin{array}{l}0.28(0.10 \\
0.46)\end{array}$ & $\begin{array}{l}-0.01(-0.19 \\
0.17)\end{array}$ & $\begin{array}{l}0.00(-0.18 \\
0.18)\end{array}$ \\
\hline Pr> chisq & 0.24 & $<0.01$ & 0.03 & 0.86 & $<0.01$ & 0.94 & 0.99 \\
\hline \multicolumn{8}{|l|}{$\begin{array}{l}\text { Learning } \\
\text { materials }\end{array}$} \\
\hline $\begin{array}{l}\text { Ordinal } \\
\text { categorical }\end{array}$ & $\begin{aligned} & 0.01(-0.18 \\
156 & 0.20)\end{aligned}$ & $\begin{array}{l}0.06(-0.14 \\
0.26)\end{array}$ & $\begin{array}{l}0.11(-0.08, \\
0.31)\end{array}$ & $\begin{array}{l}0.16(-0.03, \\
0.36)\end{array}$ & $\begin{array}{l}0.06(-0.13, \\
0.26)\end{array}$ & $\begin{array}{l}0.16(-0.03, \\
0.35)\end{array}$ & $\begin{array}{l}0.22(0.04 \\
0.41)\end{array}$ \\
\hline Pr> chisq & 0.89 & 0.54 & 0.26 & 0.10 & 0.52 & 0.10 & 0.02 \\
\hline \multicolumn{8}{|l|}{ Acceptance } \\
\hline $\begin{array}{l}\text { Ordinal } \\
\text { categorical }\end{array}$ & $\begin{array}{ll} & 0.07(-0.13 \\
156 & 0.26)\end{array}$ & $\begin{array}{l}-0.03(-0.24 \\
0.17)\end{array}$ & $\begin{array}{l}0.01(-0.19 \\
0.21)\end{array}$ & $\begin{array}{l}0.03(-0.17 \\
0.23)\end{array}$ & $\begin{array}{l}0.18(-0.02 \\
0.37)\end{array}$ & $\begin{array}{l}0.10(-0.10 \\
0.29)\end{array}$ & $\begin{array}{l}0.11(-0.08 \\
0.30)\end{array}$ \\
\hline Pr>chisq & 0.51 & 0.77 & 0.95 & 0.79 & 0.08 & 0.32 & 0.25 \\
\hline \multicolumn{8}{|l|}{ Variety } \\
\hline $\begin{array}{l}\text { Ordinal } \\
\text { categorical }\end{array}$ & $\begin{array}{ll} & 0.00(-0.20 \\
156 & 0.19)\end{array}$ & $\begin{array}{l}0.21(0.00 \\
0.42)\end{array}$ & $\begin{array}{l}0.16(-0.04 \\
0.36)\end{array}$ & $\begin{array}{l}0.13(-0.08, \\
0.33)\end{array}$ & $\begin{array}{l}0.22(0.03 \\
0.42)\end{array}$ & $\begin{array}{l}0.09(-0.11 \\
0.29)\end{array}$ & $\begin{array}{l}0.10(-0.09 \\
0.30)\end{array}$ \\
\hline Pr>chisq & 0.97 & 0.05 & 0.12 & 0.22 & 0.03 & 0.37 & 0.31 \\
\hline
\end{tabular}

${ }^{1}$ Higher factor scores indicate better outcomes (e.g., less impulsivity/internalizing, lower levels of anxiety/internalizing behaviors, higher verbal intelligence)

Characteristics presented for all participants who were included in the factor analysis, where data is available 


\section{APPENDIX 5 FREQUENTIST ASSOCIATIONS BETWEEN OPS AND NEURODEVELOPMENTAL FACTOR SCORES, WITH AND WITHOUT ADJUSTMENT FOR CO-EXPOSURES}

\begin{tabular}{|c|c|c|c|c|}
\hline & \multicolumn{2}{|c|}{$\sum \mathrm{DMPs}(\beta, 95 \% \mathrm{Cl})$} & \multicolumn{2}{|c|}{$\sum \operatorname{DEPs}(\beta, 95 \% \mathrm{Cl})$} \\
\hline & $\begin{array}{l}\text { No co-exposure } \\
\text { adjustment } \\
(n=157)\end{array}$ & $\begin{array}{l}\text { Co-exposure } \\
\text { adjustment }^{+} \\
(n=141)\end{array}$ & $\begin{array}{c}\text { No co-exposure } \\
\text { adjustment }(n=156)\end{array}$ & $\begin{array}{c}\text { Co-exposure } \\
\text { adjustment }(n=141)\end{array}$ \\
\hline Impulsivity \& & $0.03(-0.11,0.17)$ & $-0.02(-0.18,0.14)$ & $0.07(-0.10,0.23)$ & $0.11(-0.08,0.30)$ \\
\hline Externalizing & & & & \\
\hline Executive Functioning & $0.18(0.03,0.33)$ & $0.18(0.01,0.36)$ & $-0.02(-0.20,0.15)$ & $-0.11(-0.31,0.10)$ \\
\hline Internalizing & $-0.16(-0.30,-0.02)$ & $-0.16(-0.31,0.00)$ & $-0.08(-0.23,0.08)$ & $-0.02(-0.20,0.15)$ \\
\hline Perceptual Reasoning & $0.05(-0.09,0.19)$ & $-0.01(-0.17,0.16)$ & $-0.01(-0.17,0.15)$ & $0.00(-0.19,0.20)$ \\
\hline Adaptability & $-0.03(-0.18,0.12)$ & $-0.01(-0.18,0.15)$ & $-0.10(-0.26,0.07)$ & $-0.11(-0.30,0.08)$ \\
\hline Processing Speed & $-0.01(-0.16,0.13)$ & $0.01(-0.16,0.18)$ & $0.04(-0.13,0.21)$ & $-0.02(-0.22,0.18)$ \\
\hline Verbal Intelligence & $0.06(-0.06,0.19)$ & $0.07(-0.07,0.21)$ & $-0.04(-0.18,0.11)$ & $-0.04(-0.21,0.13)$ \\
\hline Working Memory Index & $0.07(-0.08,0.23)$ & $0.16(0.00,0.33)$ & $-0.16(-0.33,0.02)$ & $-0.23(-0.42,-0.04)$ \\
\hline
\end{tabular}

* The frequentist beta coefficients and $95 \%$ confidence intervals are from models that include one OP at a time (DEPs alone, DMPs alone - "no co-exposure adjustment"), and also models that include both DAP measures ( $\sum$ DEPs and $\sum$ DMPs), along with the molar sum of DEHP, the molar sum of low-molecular weight phthalates (MIBP, MEP, MBP) and the individual phthalate congeners of MCPP, MBZP, as well as the individual phenol metabolites BPA, BP3, DCP25, and triclosan, and the pyrethroid metabolite 3PBA ("co-exposure adjustment"). Factor scores have been scaled so that positive scores indicate more positive outcomes and negative scores indicate more adverse outcomes HOME scores, maternal IQ, maternal education at follow-up, maternal marital status at follow-up, maternal age, maternal race/ethnicity, maternal smoking during pregnancy, maternal alcohol consumption during pregnancy, maternal canned fish consumption during pregnancy, and alcohol were included in a DAG used to derive minimally sufficient sets for each facto 


\title{
APPENDIX 6 CODE USED IN DISSERTATION
}

\author{
\#\#SAS CODE FOR CREATING FACTORS \\ ${ }^{* *}$ VARIMAX \\ proc factor data=standardize nfactors $=7$ rotate=varimax score outstat=pca 7 fuzz $=0.3$ simple msa ; \\ var block_scale code_scale matreason_scale picconcep_scale vocab_scale symsearch_scale \\ PIQ_PRI PSQ_PSI VIQ_VCI fsiq br_bri_mean br_emotional_mean br_inhibit_mean br_initiate_mean \\ br_memory_mean br_mi_mean br_monitor_mean \\ br_organization_mean br_plan_mean br_shift_mean br_gec_mean \\ adp_t_mean0123 agg_t_mean 0123 anx_t_mean0123 att_t_mean0123 hyp_t_mean0123 \\ wth_t_mean 0123 intr_t mean0123 ext t \\ dep_t_mean0123 som_t_mean0123 aty_t_mean0123 soc_t_mean0123 adpt_t_mean0123 \\ led_t mean0123 con_t_mean0123; \\ RUN; \\ proc score data=standardize score=pca_7 out=pca_7scores; \\ var block_scale code_scale matreason_scale picconcep_scale vocab_scale symsearch_scale \\ PIQ_PRI PSQ_PSI VIQ_VCI fsiq br_bri_mean br_emotional_mean br_inhibit_mean br_initiate_mean \\ br_memory_mean br_mi_mean br_monitor_mean \\ br_organization_mean br_plan_mean br_shift_mean br_gec_mean \\ adp_t_mean0123 agg_t_mean 0123 anx_t_mean0123 att_t_mean0123 hyp_t_mean0123 \\ wth_t_mean0123 intr_t_mean0123 ext_t_mean0123 bsi_t_mean0123 \\ dep_t_mean0123 som_t_mean0123 aty_t_mean0123 soc_t_mean0123 adpt_t_mean0123 \\ led_t_mean0123 con_t_mean0123; \\ run; \\ *********** PROMAX ROTATED FACTORS;
}

proc factor data=standardize nfactors $=\mathbf{7}$ rotate=promax score outstat=promax fuzz=0.3 simple msa ; var block_scale code_scale matreason_scale picconcep_scale vocab_scale symsearch_scale PIQ_PRI PSQ_PSI VIQ_VCI fsiq br_bri_mean br_emotional_mean br_inhibit_mean br_initiate_mean br_memory_mean br_mi_mean br_monitor_mean

br_organization_mean br_plan_mean br_shift_mean br_gec_mean

adp_t_mean0123 agg_t_mean0123 anx_t_mean0123 att_t_mean0123 hyp_t_mean0123

wth_t_mean0123 intr_t_mean0123 ext_t_mean0123 bsi_t_mean0123

dep_t_mean0123 som_t_mean0123 aty_t_mean0123 soc_t_mean0123 adpt_t_mean0123

led_t_mean0123 con_t_mean0123;

RUN;

proc score data=standardize score=promax out=promax_scores type='pattern';

var block_scale code_scale matreason_scale picconcep_scale vocab_scale symsearch_scale

PIQ_PRI PSQ_PSI VIQ_VCI fsiq br_bri_mean br_emotional_mean br_inhibit_mean br_initiate_mean br_memory_mean br_mi_mean br_monitor_mean

br_organization_mean br_plan_mean br_shift_mean br_gec_mean

adp_t_mean0123 agg_t_mean0123 anx_t_mean0123 att_t_mean0123 hyp_t_mean0123

wth_t_mean0123 intr_t_mean0123 ext_t_mean0123 bsi_t_mean0123

dep_t_mean0123 som_t_mean0123 aty_t_mean0123 soc_t_mean0123 adpt_t_mean0123

led_t_mean0123 con_t_mean0123;

run;

\#\#WINBUGS CODE, shrinking to mean of 0 and sd of 1

dedp_nm_I_LN[i] djl.dnorm.trunc(-2.5197724, 0.37500623, -500, -0.79208)

detp_nm_I_LN[i] djl.dnorm.trunc(2.33299, $1.40313628,-500,-0.004885072)$

dep_nm_I_LN[i] djl.dnorm.trunc(0.53881184, 0.1827317,-500, -0.085870945) 
dmp_nm_I_LN[i] djl.dnorm.trunc $(2.61715,0.42574248,-500,0.115065123)$

dmtp_nm_I_LN[i] djl.dnorm.trunc(3.2244, 0.39832273,-500, -0.004885072)

dmdp_nm_I_LN[i] $\sim$ djl.dnorm.trunc $(0.71069863,0.50155078,-500,-0.273969564)$

\#\#calculating DAPS sum

\#\#ALL IN NANOMOLES

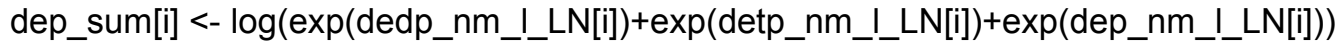

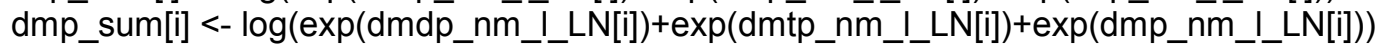

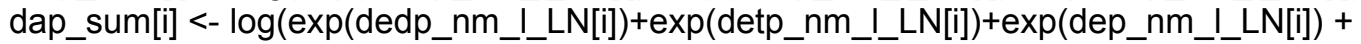

$\exp (\mathrm{dmdp}$ nm_I_LN[i])+exp(dmtp_nm_I_LN[i])+exp(dmp_nm_I_LN[i]))

dedp_out[i] <-dedp_nm_I_LN[i]

detp_out[i] <-detp_nm___LN[i]

dep_out[i] <-dep_nm_I_LN[i]

dmdp_out[i] <-dmdp_nm_I_LN[i]

dmtp_out[i] <-dmtp_nm_I_LN[i]

dmp_out[i]<-dmp_nm_I_LN[i]

dep_sum_out[i] <- dep_sum[i]

dmp_sum_out[i] <-dmp_sum[i]

\#means,sds of daps -- exported the DAPS with the values below LOD imputed, incl metabolites \# and summary measures, then merged with variables that had actual measured value, took means and got:

\#dep $-5.0416379,1.7373222$

\#detp -4.7876878, 1.0523500

\#dedp -7.0713732, 0.6675843

\#dmp -4.3240917, 1.4910056

\#dmdp -6.0450593, 1.2687206

\#dmtp -3.6438471, 1.5411503

\#depsum -3.8359428, 1.1403774

\#dmpsum -2.9558573, 1.3550287

\#dapsum -2.4316833, 1.1579961

\#\#calculate cratio

\# predict creatinine from variables

Increat[i] $\sim$ dnorm (mu.creat[i], tau.creat)

mu.creat[i] <- alpha.creat +

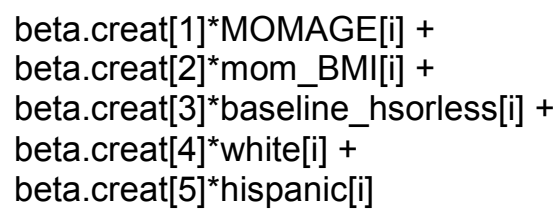

\#creatinine ratio

cratio[i] <- $(\exp ($ Increat[i] $)) /(\exp ($ mu.creat $[i]))$

\#creatinine correct, log metabolites

cratio_dep[i] <- log(exp(dep_sum[i])/cratio[i])

cratio_dmp[i] <- log(exp(dmp_sum[i])/cratio[i])

cratio_dap[i] <- log(exp(dap_sum[i]) /cratio[i]) 
cratio_dep_out[i] <- cratio_dep[i]

cratio_dmp_out[i] <-cratio_dmp[i]

cratio_dap_out[i]<- cratio_dap[i]

\#standardize cratios

\#cratio_dapz[i] <-

cratio_depz[i] <- (cratio_dep[i] - 3.042524)/ 1.15099

cratio_dmpz[i] <- (cratio_dmp[i] - 3.837248)/1.6048

\#standardize dmpsum

dmp_sumz[i] <- (dmp_sum[i] - 3.3096552) / 1.267953

dep_sumz[i] <- (dep_sum[i] - 2.49507)/1.144497

Standard_scorez[i] <- (Standard_score[i] - 93.8 )/14.3

\#\#\#OTHER VALUES

\#\#PTHALATES

mbp_log[i] djl.dnorm.trunc(3.58629, 0.788910677,-500,-0.3566749)

mbzp_log[i] djl.dnorm.trunc $(2.73489,0.48863088,-500,-0.5516476)$

mcpp_log[i] djl.dnorm.trunc(1.16315, 0.94624838,-500,-1.6094379)

mecpp_log[i] djl.dnorm.trunc(3.55535,0.47212255,-500,0.0953102)

mehhp_log[i] djl.dnorm.trunc $(2.97041,0.33366341,-500,-0.9162907)$

mehp_log[i] djl.dnorm.trunc(1.79176,0.36260978,-500,-0.1053605)

meohp_log[i] djl.dnorm.trunc $(2.85647,0.36732536,-500,0.0953102)$

mep_log[i] djl.dnorm.trunc(5.52228,0.43680145,-500,0.1151128)

mibp_log[i] djl.dnorm.trunc(1.82455,0.96914124,-500,-1.2039728)

\#other xenobiotics:

\#no non detects for dcp25 or bp3

bpa_In[i] djl.dnorm.trunc $(0.26236,0.97112055,-500,-0.9162907)$

trcs_In[i] djl.dnorm.trunc(2.37955, 0.22015845, -500, 0.80)

\#phenols and pyrethroids list:

\#bpa_In dcp24_In dcp25_In bp3_In trcs_In tcp245_In pba_bin dcca_bin

\# Calculate dehp sum

dehp_log[i] <- $\log \left(\left(\exp \left(\operatorname{mehp} \_\log [i]\right) / 278.34348\right)+(\exp (\operatorname{mehhp} \log [i]) / 294.34288)\right.$

$+(\exp ($ meohp_log[i] $) / 292.327)+(\exp ($ mecpp_log[i] $) / 308.3264))$

lophth_log[i] <- $\log \left(\left(\exp \left(m b p \_l o g[i]\right) / 222.24\right)+\left(\exp \left(\operatorname{mibp} \_\log [i]\right) / 222.26\right)+\right.$ $\exp ($ mep_log[i] $) / 94.184)$

\# Standardize metabolites to mean and SD of observed distributions

mbpz[i] $<-\left(m b p \_l o g[i]-3.58629\right) / 1.12572449$

mbzpz[i] <-(mbzp_log[i]- 2.73489$) / 1.430571429$

mcppz[i] <-(mcpp_log[i]- 1.16315)/1.028010204

mecppz[i] <-(mecpp_log[i]- 3.55535)/1.455367347

mehhpz[i] <-(mehhp_log[i]- 2.97041)/1.731193878

mehpz[i] <-(mehp_log[i]- 1.79176)/1.660658163

meohpz[i] <-(meohp_log[i] -2.85647)/1.649964286

mepz[i] $<-($ mep_log[i] - 5.52228)/1.513066327

mibpz[i] <-(mibp_log[i]- 1.82455)/1.015795918 
bpaz[i]<- (bpa_In[i]- 0.26236)/1.014760204

trcsz[i]<-(trcs_In[i] - 2.37955)/2.131239796

bp3z[i] <- $(b p 3 \ln [i]-2.4019948) / 2.0856463$

dcp25z[i] <- $($ dcp25_In[i] - 4.1312213)/1.5600545

dehpz[i] <- (dehp_log[i] - 0.0106402)/0.9915109

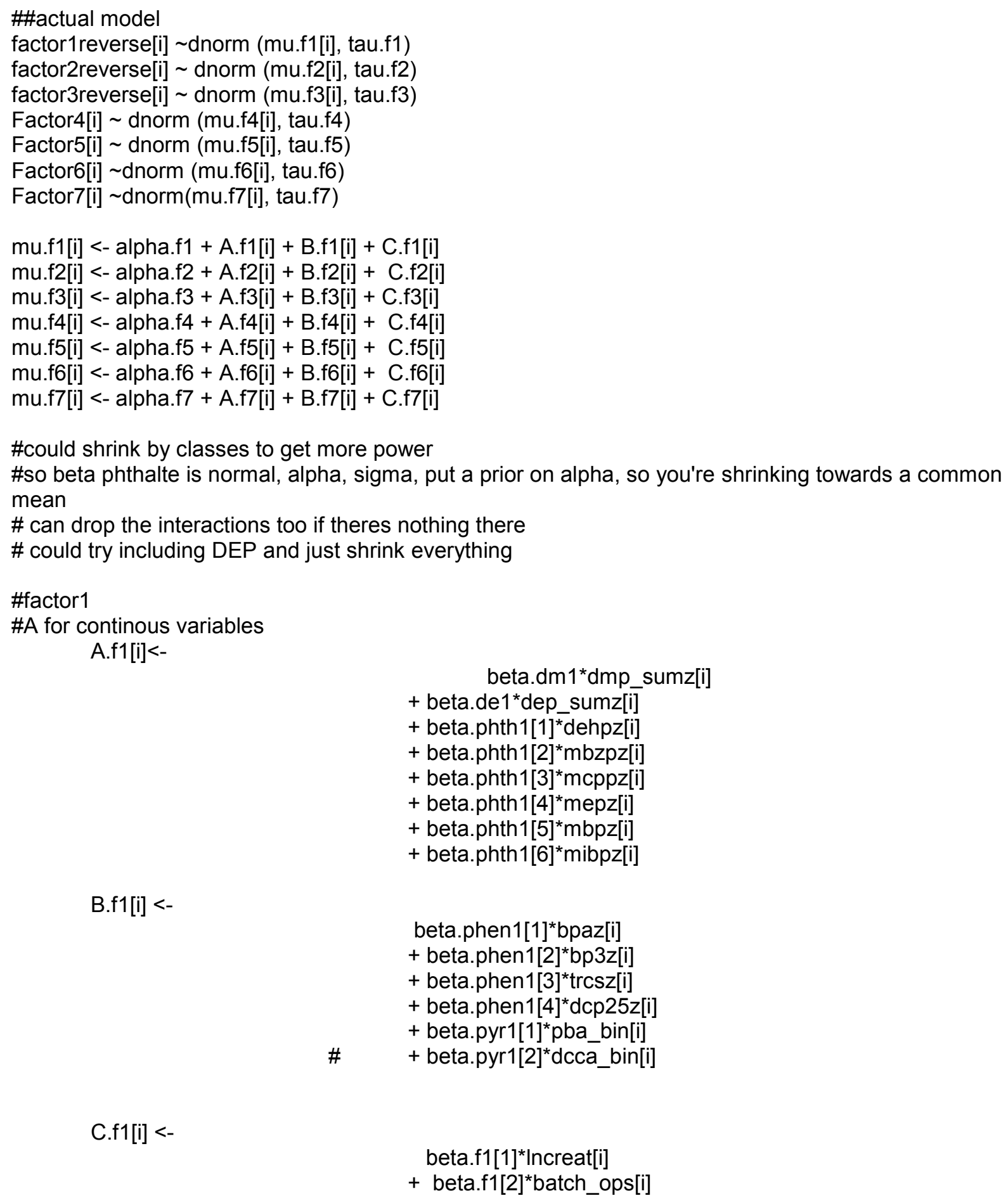

B.f1[i] <-

$$
\begin{aligned}
& \text { beta.phen } 1[1]^{*} \text { bpaz[i] } \\
& \text { + beta.phen } 1[2]^{*} b p 3 z[i] \\
& \text { + beta.phen1[3] } \operatorname{trcsz}[\mathrm{i}] \\
& \text { + beta.phen1[4] }]^{*} d c p 25 z[i] \\
& \text { + beta.pyr1[1]*pba_bin[i] } \\
& \text { \# + beta.pyr1[2] }{ }^{*} \text { dcca_bin[i] }
\end{aligned}
$$

C.f1[i] <-

$$
\text { beta.f1[1] Increat[i] }
$$$$
\text { + beta.f1[2]"batch_ops[i] }
$$ 


\author{
+ beta.f1[3] ${ }^{*}$ single_f[i] \\ +beta.f1[4] ${ }^{\star}$ SmokePreg[i] \\ +beta.f1[5] ${ }^{*}$ Male[i] \\ +beta.f1[6]*home_yr1yr2[i] \\ +beta.f1[7]*spanish[i] \\ +beta.f1[8]*hispanic[i] \\ +beta.f1[9]*married_f[i]
}

\#factor2

A.f2[i] $<-$

$$
\begin{aligned}
& \text { beta.dm2*dmp_sumz[i] } \\
& \text { +beta.de2*dep_sumz[i] } \\
& \text { + beta.phth2[1] }{ }^{\star} \text { dehpz[i] } \\
& \text { + beta.phth2[2] }{ }^{*} \mathrm{mbzpz}[\mathrm{i}] \\
& \text { + beta.phth2[3] }{ }^{*} \mathrm{mcppz}[\mathrm{i}] \\
& \text { + beta.phth2[4] }{ }^{*} \operatorname{mepz}[\mathrm{i}] \\
& \text { + beta.phth2[5] }{ }^{*} \mathrm{mbpz}[\mathrm{i}] \\
& \text { + beta.phth2[6] }{ }^{\star} \text { mibpz[i] }
\end{aligned}
$$

B.f2[i] <-

C.f2[i] <-

$$
\begin{aligned}
& \text { beta.phen2[1]*bpaz[i] } \\
& \text { + beta.phen2[2]*bp3z[i] } \\
& \text { + beta.phen2[3] }]^{\star} \operatorname{trcsz}[\mathrm{i}] \\
& \text { + beta.phen2[4] }{ }^{*} \mathrm{dcp} 25 z[i] \\
& \text { + beta.pyr2[1] }]^{*} \text { pba_bin[i] } \\
& \text { \# + beta.pyr2[2] }{ }^{*} \text { dcca_bin[i] }
\end{aligned}
$$

beta.f2[1]* Increat[i]

+ beta.f2[2]*batch_ops[i]

+ beta.f2[3] ${ }^{*}$ organization_yr1yr2_3[i]

+beta.f2[4]*involvement_yr1yr2_3[i]

+beta.f2[5] ${ }^{*}$ MOMAGE[i]

+beta.f2[6] ${ }^{*}$ Standard_scorez[i]

+beta.f2[7] ${ }^{*}$ carer_bach[i]

+beta.f2[8] ${ }^{*}$ white[i]

+beta.f2[9]*hispanic[i]

+beta.f2[10] ${ }^{*}$ SmokePreg[i]

+beta.f2[11] Male[i]

+beta.f2[12] ${ }^{*}$ carer_hs[i]

\#factor3

A. $f 3[i]<-$

$$
\begin{aligned}
& \text { beta.dm3*dmp_sumz[i] } \\
& \text { + beta.de }{ }^{*} \text { dep_sumz[i] } \\
& \text { + beta.phth3[1] }{ }^{*} \operatorname{dehpz}[i] \\
& \text { + beta.phth3[2] }{ }^{*} \mathrm{mbzpz}[\mathrm{i}] \\
& \text { + beta.phth3[3] }{ }^{*} \mathrm{mcppz}[\mathrm{i}] \\
& \text { + beta.phth3[4] }{ }^{*} \operatorname{mepz}[i] \\
& \text { + beta.phth3[5] }{ }^{*} \mathrm{mbpz}[\mathrm{i}] \\
& \text { + beta.phth3[6] }{ }^{*} \operatorname{mibpz}[i]
\end{aligned}
$$


B.f3[i] <-

C.f3[i] <-

beta.phen3[1]*bpaz[i]

+ beta.phen3[2]*bp3z[i]

+ beta.phen3[3]*trcsz[i]

+ beta.phen3[4] $]^{*} d c p 25 z[i]$

+ beta.pyr3[1]*pba_bin[i]

+ beta.pyr3[2]*dcca_bin[i]

beta.f3[1]*Increat[i]

+ beta.f3[2]*batch_ops[i]

+ beta.f3[3]*organization_yr1yr2_3[i]

+beta.f3[4] $]^{*}$ carer_hs[i]

\#factor4

A.f4[i] $<-$

$$
\begin{aligned}
& \text { beta.dm4*dmp_sumz[i] } \\
& \text { + beta.de } 4^{*} \text { dep_sumz[i] } \\
& \text { + beta.phth4[1] }{ }^{*} \text { dehpz[i] } \\
& \text { + beta.phth4[2] }{ }^{*} \mathrm{mbzpz}[\mathrm{i}] \\
& \text { + beta.phth4[3] }{ }^{*} \mathrm{mcppz}[\mathrm{i}] \\
& \text { + beta.phth4[4] }{ }^{*} \operatorname{mepz}[i] \\
& \text { + beta.phth4[5] }{ }^{*} \mathrm{mbpz}[\mathrm{i}] \\
& \text { + beta.phth4[6] }{ }^{*} \text { mibpz[i] }
\end{aligned}
$$

B.f4[i] <-

C.f4[i]<-

beta.phen4[1] $]^{*}$ bpaz[i]

+ beta.phen4[2]*bp3z[i]

+ beta.phen4[3] $]^{*} \operatorname{trcsz}[i]$

+ beta.phen4[4] $]^{*} \mathrm{dcp} 25 z[\mathrm{i}]$

+ beta.pyr4[1]pba_bin[i]

\# + beta.pyr4[2] $]^{*}$ dcca_bin[i]

beta.f4[1] $]^{*}$ Increat[i]

+ beta.f4[2]*batch_ops[i]

+beta. $f 4[3]^{*}$ white[i]

+beta. $f 4[4]^{*}$ single_f[i]

+beta.f4[5]* MOMĀGE[i]

+beta.f4[6] $]^{\star}$ Standard_scorez[i]

+beta.f4[7] ${ }^{*}$ organization_yr1yr2_3[i]

+beta.f4[8] ${ }^{*}$ carer_bach[i]

+beta.f4[9]*involvement_yr1yr2_3[i]

+beta.f4[10]*alcohol_cat1 [i]

+beta.f4[11]*spanish[i]

+beta.f4[12] ${ }^{*}$ examiner2[i]

+beta.f4[13] ${ }^{*}$ examiner3[i]

+beta. $f 4[14]^{*}$ examiner $4[i]$

+beta. $f 4[15]^{*}$ examiner5[i]

+beta. $f 4[16]^{*}$ canfish 1 xweek[i]

\#factor5

A.f5[i] $<-$

$$
\begin{aligned}
& \text { beta.dm5 } 5^{*} \mathrm{dmp} \text { _sumz[i] } \\
& \text { + beta.de } 5^{*} \text { dep_sumz[i] } \\
& \text { + beta.phth5[1] }]^{*} \operatorname{dehpz}[i] \\
& \text { + beta.phth5[2] }{ }^{*} \mathrm{mbzpz}[\mathrm{i}]
\end{aligned}
$$




$$
\begin{aligned}
& \text { + beta.phth5[3] }{ }^{*} \mathrm{mcppz}[\mathrm{i}] \\
& \text { + beta.phth5[4] }{ }^{*} \operatorname{mepz}[i] \\
& \text { + beta.phth5[5] }{ }^{*} \mathrm{mbpz}[\mathrm{i}] \\
& \text { + beta.phth5[6] }{ }^{*} \operatorname{mibpz}[i]
\end{aligned}
$$

B.f5[i] <-

C.f5[i] <-

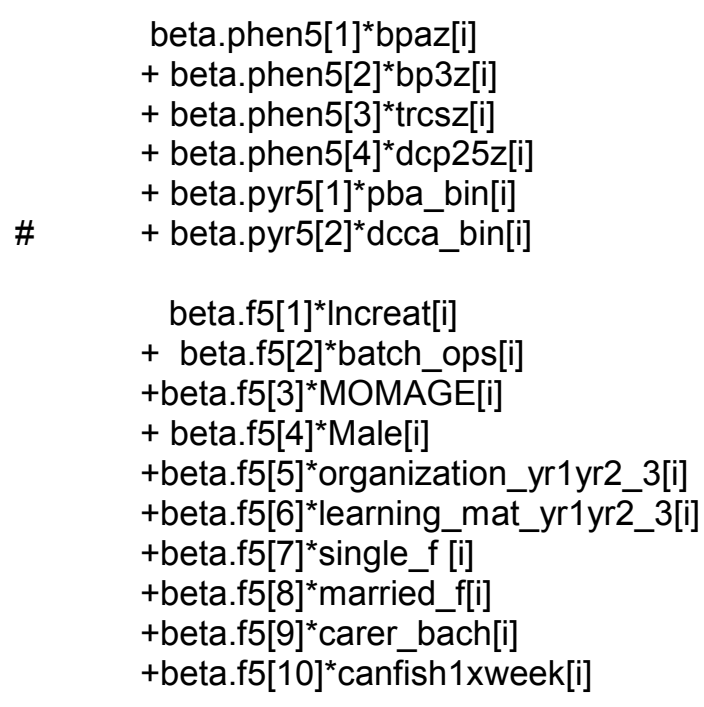

\#factor6

A.f6[i] $<-$

$$
\begin{aligned}
& \text { beta.dm6*dmp_sumz[i] } \\
& \text { +beta.de6 }{ }^{*} \text { dep_sumz[i] } \\
& \text { + beta.phth6[1] }]^{*} \operatorname{dehpz}[i] \\
& \text { + beta.phth6[2] }{ }^{*} \mathrm{mbzpz}[\mathrm{i}] \\
& \text { + beta.phth6[3] }{ }^{*} \mathrm{mcppz}[\mathrm{i}] \\
& \text { + beta.phth6[4] }{ }^{*} \operatorname{mepz}[\mathrm{i}] \\
& \text { + beta.phth6[5] }{ }^{*} \mathrm{mbpz}[\mathrm{i}] \\
& \text { + beta.phth6[6] }{ }^{*} \operatorname{mibpz}[i]
\end{aligned}
$$

B.f6[i] <-

C.f6[i] <-

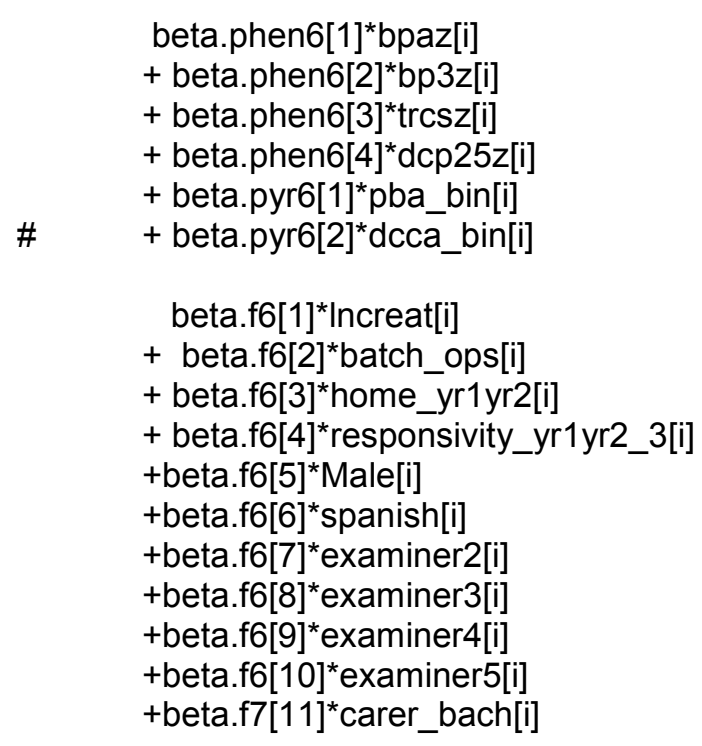


\#factor7

A.f7[i] $<-$

$$
\begin{aligned}
& \text { beta.dm7*dmp_sumz[i] } \\
& \text { + beta.de } 7^{*} \text { dep_sumz[i] } \\
& \text { + beta.phth7[1] }{ }^{*} \operatorname{dehpz}[\mathrm{i}] \\
& \text { + beta.phth7[2] }{ }^{*} \mathrm{mbzpz}[\mathrm{i}] \\
& \text { + beta.phth7[3] }{ }^{*} \mathrm{mcppz}[\mathrm{i}] \\
& \text { + beta.phth7[4] }{ }^{*} \operatorname{mepz}[\mathrm{i}] \\
& \text { + beta.phth7[5] }{ }^{*} \mathrm{mbpz}[\mathrm{i}] \\
& \text { + beta.phth7[6] }{ }^{*} \operatorname{mibpz}[\mathrm{i}]
\end{aligned}
$$

B.f7[i] <-

beta.phen7[1] $]^{*}$ ppaz[i]

+ beta.phen7[2]*bp3z[i]

+ beta.phen7[3]*trcsz[i]

+ beta.phen7[4] $]^{*} \mathrm{dcp} 25 z[\mathrm{i}]$

+ beta.pyr7[1] $]^{*}$ pba_bin[i]

C.f7[i] <-

+ beta.pyr7[2]*dcca_bin[i]

$$
\begin{aligned}
& \text { beta.f7[1]*Increat[i] } \\
& \text { + beta.f7[2]*batch_ops[i] } \\
& \text { + beta.f7[3]*MOMAGE[i] } \\
& \text { +beta.f7[4]*single_f[i] } \\
& \text { +beta.f7[5]*learning_mat_yr1yr2_3[i] } \\
& \text { +beta.f7[6] }{ }^{*} \text { carer_hs[i] } \\
& + \text { beta.f7[7] }{ }^{*} \text { Standard_score[i] } \\
& \text { +beta.f7[8]*married_f[i] } \\
& \text { +beta.f7[9] }{ }^{*} \text { carer_bach[i] } \\
& \text { +beta.f7[10]*spanish[i] } \\
& \text { +beta.f7[11] }{ }^{*} \text { white[i] } \\
& \text { +beta.f7[12] }{ }^{*} \text { examiner4[i] } \\
& \text { + beta.f7[13]*alcohol_cat1[i] } \\
& \text { +beta.f7[14] }{ }^{*} \text { canfish } 1 \text { xweek[i] } \\
& \text { +beta.f7[15] }{ }^{*} \text { examiner5[i] } \\
& \text { +beta.f7[16] }{ }^{*} \text { examiner2[i] } \\
& \text { +beta.f7[17] }{ }^{*} \text { examiner3[i] }
\end{aligned}
$$

\# missing variables: home scores, alcohol_cat1, stnadard_score

home_yr1yr2[i] dnorm (homemu[i], tau.home)

homemu[i] <- alpha.home + A.home[i] +B.home[i]

A.home $[i]<-$ beta.home $[1]^{*}$ canfish1xweek $[i]+$ beta.home[2] ${ }^{*}$ white[i] + beta.home[3] $]^{*}$ carer_bach[i] + beta.home[4] ${ }^{\star}$ factor 1 reverse[i]+ beta.home[5] factor2reverse[i]+

B.home $[i]<-$ beta.home[6] ${ }^{\star}$ factor3reverse[i]

beta.home[7] ${ }^{*}$ Male[i] + beta.home[8] ${ }^{*}$ SmokePreg[i] + beta.home[9] ${ }^{*}$ Standard_scorez[i] + 


\begin{abstract}
beta.home[10] ${ }^{*}$ Factor4[i]+ beta.home[11] ${ }^{\star}$ Factor5[i]+ beta.home[12]*Factor6[i] + beta.home[13] ${ }^{\star}$ Factor7[i] + beta.home[14]*married_f[i] + beta.home[15]*hispanic[i] + beta.home[16]*MOMAGE[i]
\end{abstract}

organization_yr1yr2_3[i] dnorm (orgmu[i], tau.org) orgmu[i] <- alpha.org + A.org[i] +B.org[i]

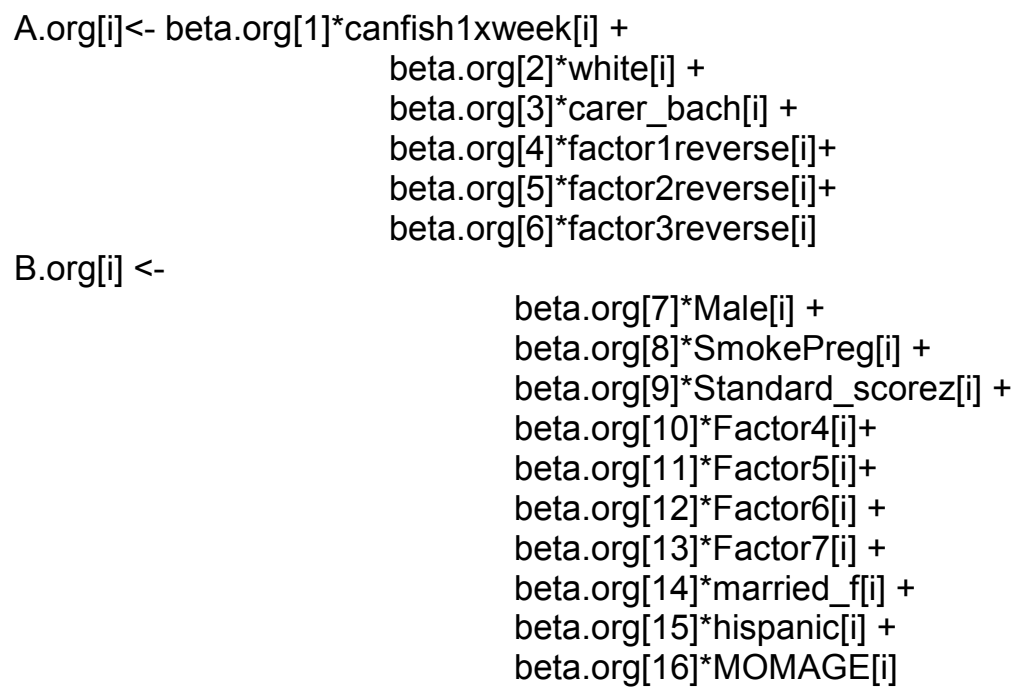

learning_mat_yr1yr2_3[i] dnorm (Immu[i], tau.Im)

Immu[i] <- alpha. Im + A. $\operatorname{Im}[i]+B \cdot \operatorname{Im}[i]$

A. $\operatorname{Im}[i]<-$ beta. $\operatorname{Im}[1]^{*}$ canfish1xweek[i] +

beta. $\operatorname{Im}[2]^{*}$ white[i] +

beta.Im[3] ${ }^{*}$ carer_bach $[i]+$

beta. $\operatorname{Im}[4]^{*}$ factor 1 reverse[i]+

beta. $\operatorname{lm}[5]^{\star}$ factor2reverse[i]+

B. $\operatorname{lm}[i]<-$

beta. $\operatorname{Im}[6]^{\star}$ factor3reverse[i]

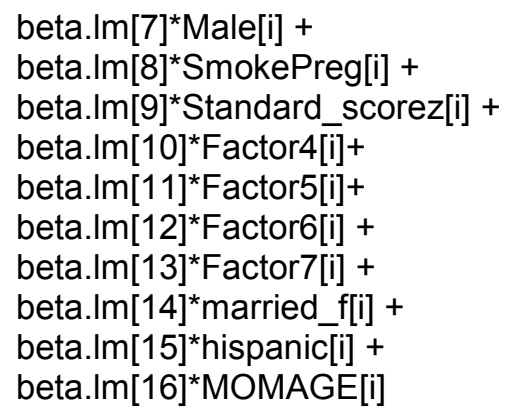

variety_yr1yr2_3[i] dnorm (vmu[i], tau.v)

vmu[i] <- alpha.v + A.v[i] +B.v [i]

A.v $[\mathrm{i}]<-$ beta.v $[1]^{*}$ canfish1xweek[i] +

beta.v $[2]^{*}$ white $[i]+$

beta.v [3] $]^{*}$ carer_bach[i] +

beta.v [4] ${ }^{\star}$ factor1 reverse[i]+

beta.v [5] ${ }^{\star}$ factor2reverse[i]+ 
B.v [i] <-

beta.v [6] ${ }^{\star}$ factor3reverse[i]

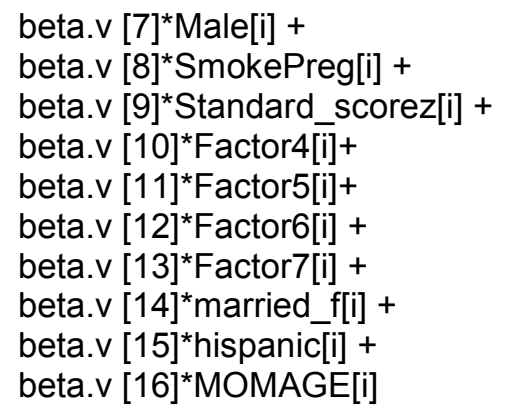

responsivity_yr1yr2_3[i] dnorm (rmu[i], tau.r)

$r m u[i]<-$ alpha.r + A.r[i] +B.r [i]

A.r $[i]<-$ beta.r[1] $]^{*}$ canfish1xweek[i] +

beta.r [2] ${ }^{*}$ white[i] +

beta.r [3] ${ }^{*}$ carer_bach[i] +

beta.r [4] ${ }^{\star}$ factor1reverse[i]+

beta.r [5] ${ }^{\star}$ factor2reverse[i]+

B.r $[i]<-$

beta.r [6] ${ }^{\star}$ factor3reverse[i]

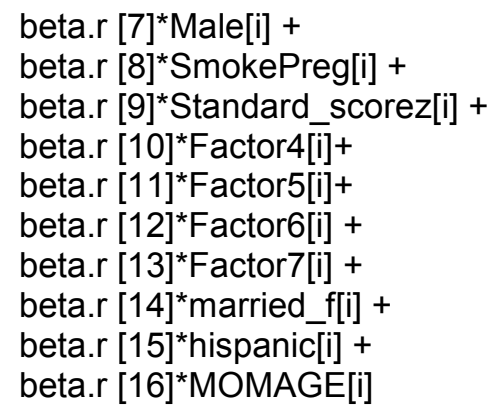

involvement_yr1yr2_3[i] dnorm (invmu[i], tau.inv)

invmu[i] <- alpha.inv + A.inv[i] +B.inv [i]

A.inv[i]<- beta.inv[1] ${ }^{*}$ canfish1xweek[i] +

$$
\begin{aligned}
& \text { beta.inv [2] }{ }^{*} \text { white [i] + } \\
& \text { beta.inv [3] }{ }^{*} \text { carer_bach[i] + } \\
& \text { beta.inv [4] }{ }^{\star} \text { factor } 1 \text { reverse[i]+ } \\
& \text { beta.inv [5] }{ }^{\star} \text { factor2reverse[i]+ }
\end{aligned}
$$

B.inv [i] <-

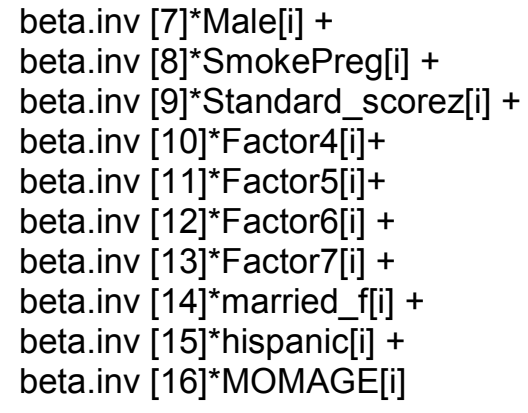

alcohol_cat1[i] dbern(p.alc[i])

logit(p.alc[i] $)<-$ alpha.alc + A.alc[i] + B.alc[i] 


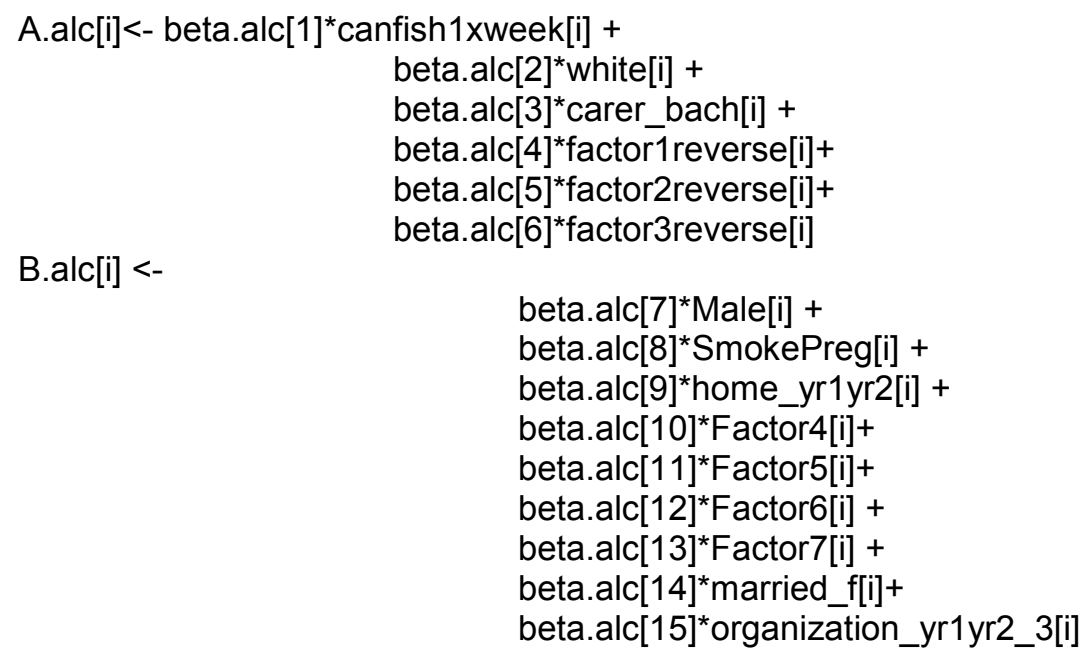

Standard_score[i] dnorm (iqmu[i], tau.iq)

iqmu[i] <- alpha.iq + A.iq[i] +B.iq [i]

A.iq [i]<- beta.iq[1] ${ }^{*}$ canfish1xweek[i] +

beta.iq [2] ${ }^{*}$ white[i] +

beta.iq [3] ${ }^{*}$ carer_bach[i] +

beta.iq [4] ${ }^{\star}$ factor 1 reverse[i]+

beta.iq [5] ${ }^{\star}$ factor2reverse[i]+

B.iq [i] $<-$

beta.iq [6] ${ }^{\star}$ factor3reverse[i]

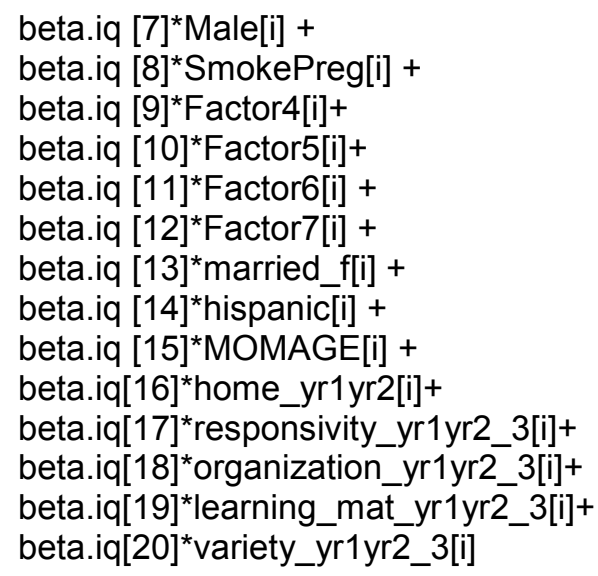

mom_BMI[i] dnorm (bmimu[i], tau.bmi)

bmimu[i] <- alpha.bmi + A.bmi[i] +B.bmi [i]

A.bmi [i]<- beta.bmi[1] ${ }^{*}$ canfish1xweek[i] +

beta.bmi [2] ${ }^{*}$ white[i] +

beta.bmi [3] ${ }^{*}$ carer_bach[i] +

beta.bmi [4]*factor1 1 reverse[i]+

beta.bmi [5] ${ }^{\star}$ factor2reverse[i]+

B.bmi $[i]<-$

beta.bmi [6] ${ }^{\star}$ factor3reverse[i]

beta.bmi [7]*Male[i] +

beta.bmi [8] ${ }^{*}$ SmokePreg[i] +

beta.bmi [9]*Factor4[i]+ 


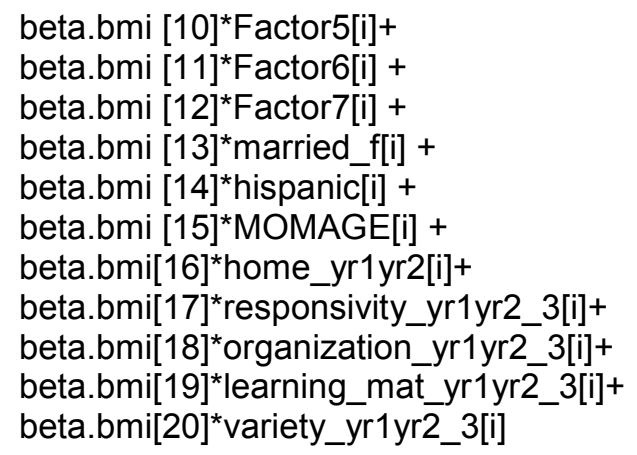




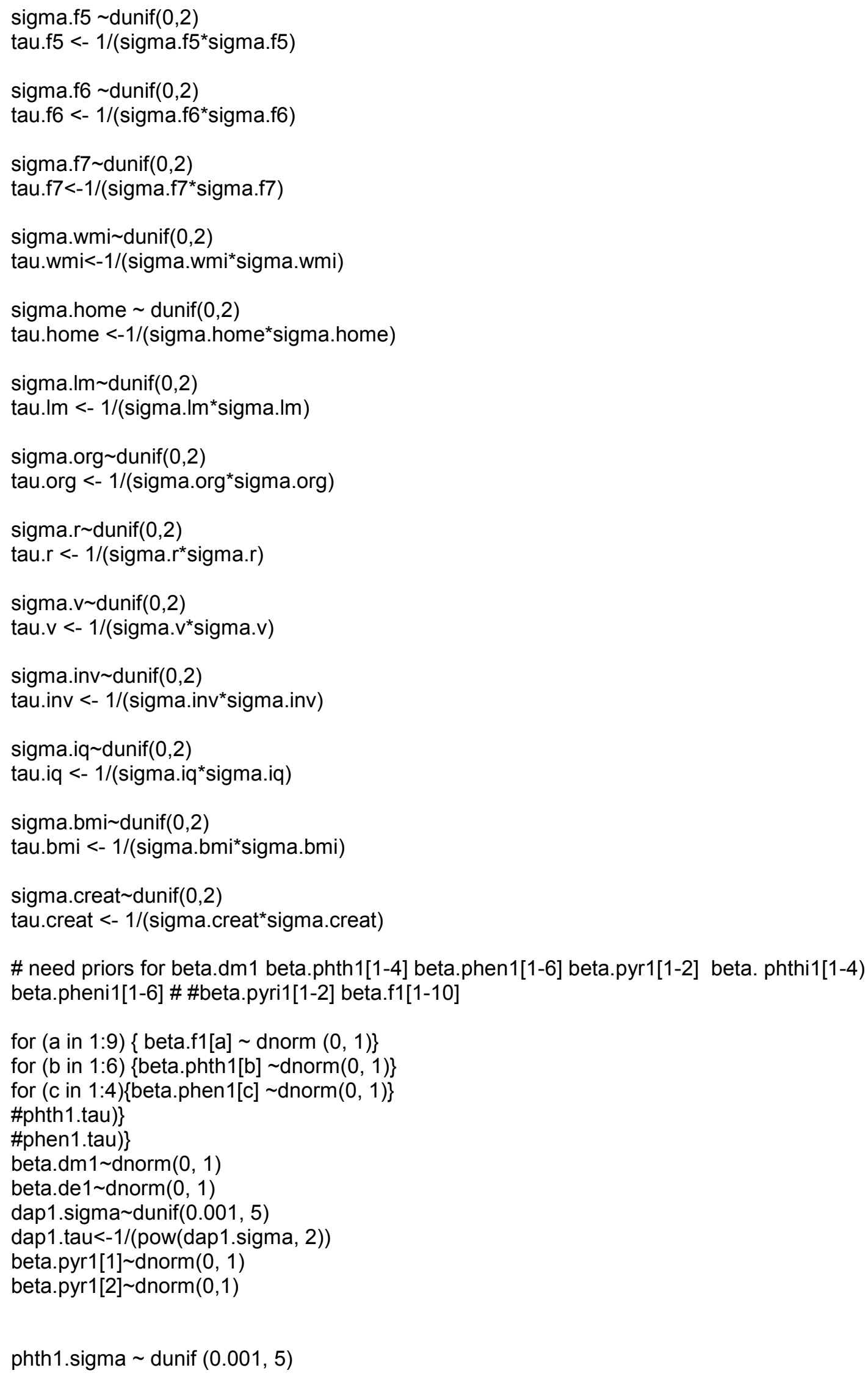


phth1.tau<-1/(pow(phth1.sigma, 2))

phen1.sigma dunif $(0.001,5)$

phen1.tau<-1/(pow(phen1.sigma,2))

for $(\mathrm{f}$ in 1:12) $\{$ beta.f2[f] dnorm $(0,1)\}$

for (g in 1:6) \{beta.phth2[g] $\operatorname{dnorm}(0,1)\}$

\#phth2.tau)\}

for (i in 1:4)\{beta.phen2[i] dnorm $(0,1)\}$

\#phen2.tau)\}

beta.dm2 dnorm $(0,1)$

beta.de2 dnorm $(0,1)$

dap2.sigma dunif $(0.001,5)$

dap2.tau<-1/(pow(dap2.sigma, 2))

beta.pyr2[1] dnorm $(0,1)$

beta.pyr2[2] dnorm $(0,1)$

phth2.sigma dunif $(0.001,5)$

phth2.tau<-1/(pow(phth2.sigma, 2))

phen2.sigma dunif $(0.001,5)$

phen2.tau<-1/(pow(phen2.sigma,2))

for $(\mathrm{k}$ in 1:4) \{ beta.f3[k] $\sim$ dnorm $(0,1)\}$

for (I in 1:6) \{beta.phth3[I] $\sim \operatorname{dnorm}(0,1)\}$

for $(\mathrm{n}$ in 1:4)\{beta.phen3[n] $\sim \operatorname{dnorm}(0,1)\}$ beta.dm3 dnorm $(0,1)$

beta.de3 dnorm $(0,1)$

beta.pyr3[1] dnorm $(0,1)$

beta.pyr3[2] dnorm $(0,1)$

dap3.sigma $\sim \operatorname{dunif}(0.001,5)$

dap3.tau<-1/(pow(dap3.sigma,2))

phth3.sigma $\sim$ dunif $(0.001,5)$

phth3.tau<-1/(pow(phth3.sigma, 2))

phen3.sigma dunif $(0.001,5)$

phen3.tau<-1/(pow(phen3.sigma,2))

for $(p$ in 1:16) $\{$ beta.f4[p] dnorm $(0,1)\}$

for (q in 1:6) \{beta.phth4[q] dnorm $(0,1)\}$

\#phth4.tau)\}

for (s in 1:4)\{beta.phen4[s] dnorm $(0,1)\}$

\# phen4.tau)\}

beta. $\mathrm{dm} 4 \sim \operatorname{dnorm}(0,1)$

beta.de4 dnorm $(0,1)$

beta.pyr4[1] dnorm $(0,1)$

beta.pyr4[2] dnorm $(0,1)$

dap4.sigma $\sim \operatorname{dunif}(0.001,5)$

dap4.tau<-1/(pow(dap4.sigma,2))

phth4.sigma $\sim$ dunif $(0.001,5)$

phth4.tau<-1/(pow(phth4.sigma, 2))

phen4.sigma dunif $(0.001,5)$

phen4.tau<-1/(pow (phen4.sigma,2)) 


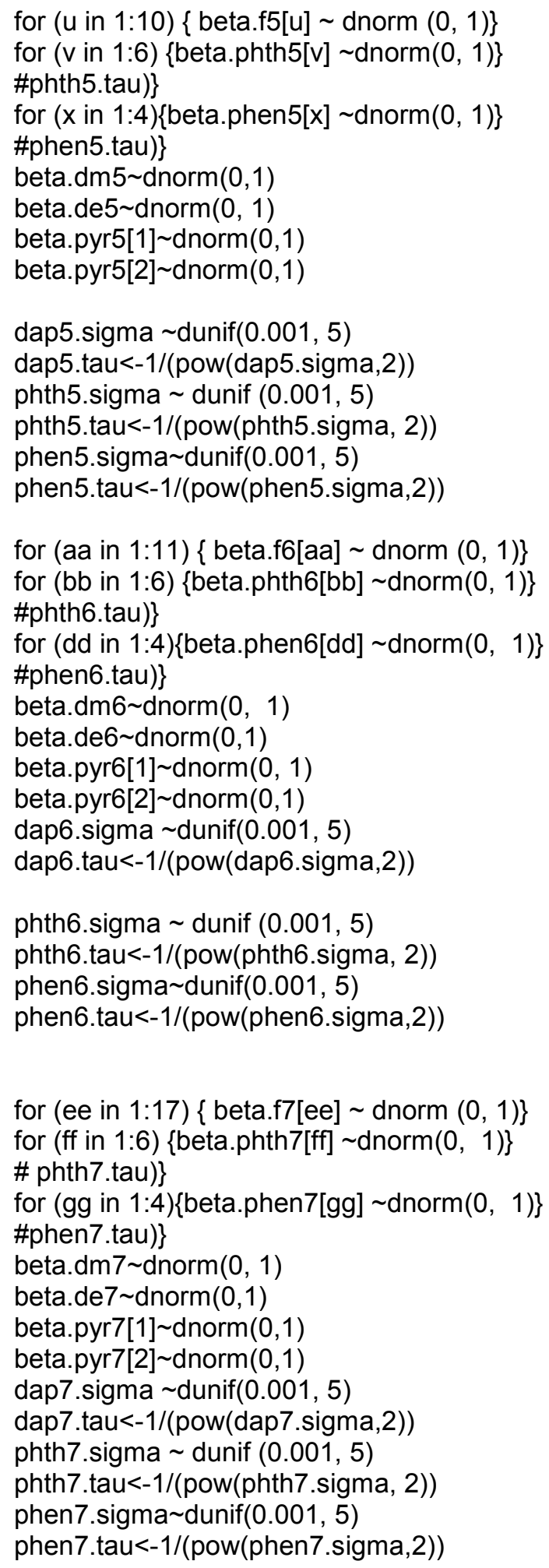

for (hh in 1:16) \{beta.org[hh] dnorm $(0,1)\}$ 
for (jj in 1:15) \{beta.alc[j] dnorm $(0,1)\}$

for $(\mathrm{kk}$ in 1:16) \{beta.Im[kk] dnorm $(0,1)\}$

for $(\mathrm{mm}$ in 1:16) \{beta.v[mm] $\sim \operatorname{dnorm}(0,1)\}$

for $(\mathrm{nn}$ in 1:16) \{beta.r[nn] dnorm $(0,1)\}$

for (oo in 1:16) \{beta.home[oo] dnorm $(0,1)\}$

for (pp in 1:16) \{beta.inv[pp] $\sim \operatorname{dnorm}(0,1)\}$

for (ii in 1:20) \{beta.iq[ii] dnorm $(0,1)\}$

for (uu in 1:5) \{beta.creat[uu] dnorm $(0,1)\}$

for $(v v$ in 1:20) \{beta.bmi[vv] dnorm $(0,1)\}$

\} 


\section{REFERENCES}

Aldridge, J. E., Meyer, A., Seidler, F. J., \& Slotkin, T. A. (2005). Alterations in central nervous system serotonergic and dopaminergic synaptic activity in adulthood after prenatal or neonatal chlorpyrifos exposure. Environmental Health Perspectives, 113(8), 1027-1031. http://doi.org/10.1289/ehp.7968

Aldridge, J. E., Seidler, F. J., \& Slotkin, T. A. (2004). Developmental exposure to chlorpyrifos elicits sex-selective alterations of serotonergic synaptic function in adulthood: Critical periods and regional selectivity for effects on the serotinin transporter, receptor subtypes, and cell signaling. Environmental Health Perspectives, 112(2), 148-155.

http://doi.org/10.1289/ehp.6713

Assini, F. L., Zanette, K. D., Brocardo, P. S., Pandolfo, P., Rodrigues, A. L. S., \& Takahashi, R. N. (2005). Behavioral effects and ChE measures after acute and repeated administration of malathion in rats. Environmental Toxicology and Pharmacology, 20(3), 443-9. http://doi.org/10.1016/j.etap.2005.05.007

Barkley, R. (1997). Behavioral Inhibition, Sustained Attention, and Executive Functions: Constructing a Unifying Theory of ADHD. Psychological Bulletin, 121(1), 65-94. http://doi.org/10.1037/0033-2909.121.1.65

Barr, D. B., Allen, R., Olsson, A. O., Bravo, R., Caltabiano, L. M., Montesano, A., ... Needham, L. L. (2005). Concentrations of selective metabolites of organophosphorus pesticides in the United States population. Environmental Research, 99(3), 314-326. http://doi.org/10.1016/j.envres.2005.03.012

Barr, D. B., Olsson, A. O., Wong, L.-Y., Udunka, S., Baker, S. E., Whitehead, R. D., ... Needham, L. L. (2010). Urinary concentrations of metabolites of pyrethroid insecticides in the general U.S. population: National health and nutrition examination survey 1999-2002. Environmental Health Perspectives, 118(6), 742-8.

Baynam, G., Walters, M., Claes, P., Kung, S., LeSouef, P., Dawkins, H., ... Goldblatt, J. (2015). Phenotyping: targeting genotype's rich cousin for diagnosis. Journal of Paediatrics and Child Health, 51(4), 381-6. http://doi.org/10.1111/jpc.12705

Beard, J. D., Umbach, D. M., Hoppin, J. A., Richards, M., Alavanja, M. C. R., Blair, A., ... Kamel, F. (2014). Pesticide Exposure and Depression among Male Private Pesticide Applicators in the Agricultural Health Study. Environmental Health Perspectives, 122(9), 984-91. http://doi.org/10.1289/ehp.1307450

Bellinger, D. C. (2013). Prenatal Exposures to Environmental Chemicals and Children's Neurodevelopment: An Update. Safety and Health at Work, 4(1), 1-11. http://doi.org/10.5491/SHAW.2013.4.1.1

Berkowitz, G. S., Obel, J., Deych, E., Lapinski, R., Godbold, J., Liu, Z., ... Wolff, M. S. (2003). Exposure to indoor pesticides during pregnancy in a multiethnic, urban cohort. Environmental Health Perspectives, 111(1), 79. 
Berkowitz, G. S., Wetmur, J. G., Birman-Deych, E., Obel, J., Lapinski, R. H., Godbold, J. H., ... Wolff, M. S. (2004). In utero pesticide exposure, maternal paraoxonase activity, and head circumference. Environmental Health Perspectives, 112(3), 388.

Bhutta, A. T., Cleves, M. A., Casey, P. H., Cradock, M. M., \& Anand, K. J. S. (2002). Cognitive and behavioral outcomes of school-aged children who were born preterm. The Journal of the American Medical Association, 288(6), 728-737. http://doi.org/10.1001/jama.288.6.728

Bodnar, L. E., Prahme, M. C., Cutting, L. E., Denckla, M. B., \& Mahone, E. M. (2007). Construct validity of parent ratings of inhibitory control. Child Neuropsychology: A Journal On Normal and Abnormal Development In Childhood and Adolescence, 13(4), 345-362. http://doi.org/10.1080/09297040600899867

Bolduc, F. V., Lau, A., Rosenfelt, C. S., Langer, S., Wang, N., Smithson, L., ... Mandhane, P. J. (2016). Cognitive Enhancement in Infants Associated with Increased Maternal Fruit Intake During Pregnancy: Results from a Birth Cohort Study with Validation in an Animal Model. EBioMedicine. http://doi.org/10.1016/j.ebiom.2016.04.025

Bouchard, M. F., Bellinger, D. C., Wright, R. O., \& Weisskopf, M. G. (2010). Attentiondeficit/hyperactivity disorder and urinary metabolites of organophosphate pesticides. Pediatrics, 125(6), e1270-e1277.

Bouchard, M. F., Chevrier, J., Harley, K. G., Kogut, K., Vedar, M., Calderon, N., ... Eskenazi, B. (2011a). Prenatal Exposure to Organophosphate Pesticides and IQ in 7-Year Old Children. Environmental Health Perspectives, 1003185(April), 1189. http://doi.org/10.1289/ehp.1003185

Bouchard, M. F., Chevrier, J., Harley, K. G., Kogut, K., Vedar, M., Calderon, N., ... Eskenazi, B. (2011b). Prenatal exposure to organophosphate pesticides and IQ in 7-year-old children. Environmental Health Perspectives, 119(8), 1189-95. http://doi.org/10.1289/ehp.1003185

Bradley, R. H., Caldwell, B. M., Rock, S. L., \& Ramey, C. T. (1989). Home environment and cognitive development in the first 3 years of life: A collaborative study involving six sites and three ethnic groups in North America. Developmental Psychology, 25(2), 217-235. http://doi.org/10.1037/0012-1649.25.2.217

Bravo, R., Caltabiano, L. M., Weerasekera, G., Whitehead, R. D., Fernandez, C., Needham, L. L., ... Barr, D. B. (2004). Measurement of dialkyl phosphate metabolites of organophosphorus pesticides in human urine using lyophilization with gas chromatography-tandem mass spectrometry and isotope dilution quantification. Journal of Exposure Science and Environmental Epidemiology, 14(3), 249-259.

Brion, M.-J., Victora, C., Matijasevich, A., Horta, B., Anselmi, L., Steer, C., ... Davey Smith, G. (2010). Maternal smoking and child psychological problems: disentangling causal and noncausal effects. Pediatrics, 126(1), e57-65. http://doi.org/10.1542/peds.2009-2754

Brocardo, P. S., Assini, F., Franco, J. L., Pandolfo, P., Müller, Y. M. R., Takahashi, R. N., ... Rodrigues, A. L. S. (2007). Zinc attenuates malathion-induced depressant-like behavior and confers neuroprotection in the rat brain. Toxicological Sciences: An Official Journal of the Society of Toxicology, 97(1), 140-8. http://doi.org/10.1093/toxsci/kfm024 
Cadoret, R. J., Langbehn, D., Caspers, K., Troughton, E. P., Yucuis, R., Sandhu, H. K., \& Philibert, R. (2003). Associations of the serotonin transporter promoter polymorphism with aggressivity, attention deficit, and conduct disorder in an adoptee population. Comprehensive Psychiatry, 44(2), 88-101.

Caldwell, B. M., \& Bradley, R. H. (1984). Administration manual: home observation for measurement of the environment. Little Rock: Department of Psychology, University of Arkansas.

Calkins, M. E., Merikangas, K. R., Moore, T. M., Burstein, M., Behr, M. A., Satterthwaite, T. D., ... Gur, R. E. (2015). The Philadelphia Neurodevelopmental Cohort: constructing a deep phenotyping collaborative. Journal of Child Psychology and Psychiatry, and Allied Disciplines, 56(12), 1356-69. http://doi.org/10.1111/jcpp.12416

Castellanos, F. X., Sonuga-Barke, E. J. S., Milham, M. P., \& Tannock, R. (2006). Characterizing cognition in attention-deficit hyperactivity disorder: beyond executive dysfunction. Trends in Cognitive Sciences, 10(3), 117-123.

Chamberlain, C., O’Mara-Eves, A., Oliver, S., Caird, J. R., Perlen, S. M., Eades, S. J., \& Thomas, J. (2013). Psychosocial interventions for supporting women to stop smoking in pregnancy. In C. Chamberlain (Ed.), Cochrane Database of Systematic Reviews. Chichester, UK: John Wiley \& Sons, Ltd. http://doi.org/10.1002/14651858.CD001055.pub4

Children, Y. M., Eskenazi, B., Marks, A. R., Bradman, A., Harley, K., Barr, D. B., ... Jewell, N. P. (2007). Research | Children 's Health Organophosphate Pesticide Exposure and Neurodevelopment in. Environmental Health Perspectives, 115(5), 792-798. http://doi.org/10.1289/ehp.9828

Christ, S. E., Holt, D. D., White, D. A., \& Green, L. (2007). Inhibitory control in children with autism spectrum disorder. Journal of Autism and Developmental Disorders, 37(6), 115565. http://doi.org/10.1007/s10803-006-0259-y

Cohen, J., Bellinger, D., Connor, W., Kris-Etherton, P., Lawrence, R., Savitz, D., ... Gray, G. (2005). A quantitative risk-benefit analysis of changes in population fish consumption. American Journal of Preventive Medicine, 29(4), 325-334.

Courchesne, E., Carper, R. A., \& Akshoomoff, N. A. (2003). Evidence of brain overgrowth in the first year of life in autism. JAMA, 290(3), 1-8. http://doi.org/10.1001/jama.290.3.337

Courchesne, E., Karns, C. M., Davis, H. R., Ziccardi, R., Carper, R. A., Tigue, Z. D., ... Courchesne, R. Y. (2001). Unusual brain growth patterns in early life in patients with autistic disorder: An MRI study. Neurology, 57(2), 245-254. http://doi.org/10.1212/WNL.57.2.245

Daniels, J. L., Longnecker, M. P., Rowland, A. S., Golding, J., \& ALSPAC. (2004). Fish intake during pregnancy and early cognitive development of offspring. Epidemiology, 15(4), 394402.

Daseking, M., Petermann, F., Tischler, T., \& Waldmann, H.-C. (2015). Smoking during pregnancy is a risk factor for executive function deficits in preschool-aged children. Geburtshilfe Und Frauenheilkunde, 75(1), 64-71. http://doi.org/10.1055/s-0034-1383419 
Diamond, A., \& Lee, K. (2011). Interventions shown to aid executive function development in children 4 to 12 years old. Science (New York, N.Y.), 333(6045), 959-64.

http://doi.org/10.1126/science.1204529

Duggan, A., Charnley, G., Chen, W., Chukwudebe, A., Hawk, R., Krieger, R. I., ... Yarborough, C. (2003). Di-alkyl phosphate biomonitoring data: assessing cumulative exposure to organophosphate pesticides. Regulatory Toxicology and Pharmacology, 37(3), 382-395.

Dunn, L. M., \& Dunn, L. M. (1997). PPVT-III: Peabody picture vocabulary test. Circle Pines, MN: American Guidance Service.

Dunson, D. B., Herring, A. H., \& Engel, S. M. (2008). Bayesian selection and clustering of polymorphisms in functionally related genes. Journal of the American Statistical Association, 103(482).

Dziuban, C. D., \& Shirkey, E. C. (1974). When is a correlation matrix appropriate for factor analysis? Some decision rules. Psychological Bulletin, 81(6), 358-361.

http://doi.org/10.1037/h0036316

Egeghy, P. P., Cohen Hubal, E. A., Tulve, N. S., Melnyk, L. J., Morgan, M. K., Fortmann, R. C., \& Sheldon, L. S. (2011). Review of pesticide urinary biomarker measurements from selected US EPA children's observational exposure studies. International Journal of Environmental Research and Public Health, 8(5), 1727-1754.

Eley, T. C., Sugden, K., Corsico, A., Gregory, A. M., Sham, P., McGuffin, P., ... Craig, I. W. (2004). Gene-environment interaction analysis of serotonin system markers with adolescent depression. Molecular Psychiatry, 9(10), 908-915. http://doi.org/10.1038/sj.mp.4001546

Engel, S. M., Berkowitz, G. S., Barr, D. B., Teitelbaum, S. L., Siskind, J., Meisel, S. J., ... Wolff, M. S. (2007). Prenatal organophosphate metabolite and organochlorine levels and performance on the Brazelton Neonatal Behavioral Assessment Scale in a multiethnic pregnancy cohort. American Journal of Epidemiology, 165(12), 1397-1404. http://doi.org/10.1093/aje/kwm029

Engel, S. M., Bradman, A., Wolff, M. S., Rauh, V. A., Harley, K. G., Yang, J. H., ... Eskenazi, B. (2015). Prenatal Organophosphorus Pesticide Exposure and Child Neurodevelopment at 24 Months: An Analysis of Four Birth Cohorts. Environmental Health Perspectives, (November 2014). http://doi.org/10.1289/ehp.1409474

Engel, S. M., \& Wolff, M. S. (2013). Causal inference considerations for endocrine disruptor research in children's health. Annual Review of Public Health, 34, 139.

Engel, S., Wetmur, J., Chen, J., Zhu, C., Barr, D. B., Canfield, R. L., \& Wolff, M. S. (2011). Prenatal exposure to organophosphates, paraoxonase 1, and cognitive development in childhood. Environmental Health Perspectives, 119(8), 1182-1188. http://doi.org/10.1289/ehp.1003183

EPA. (2016). Revised Human Health Risk Assessment on Chlorpyrifos. Retrieved from https://www.epa.gov/ingredients-used-pesticide-products/revised-human-health-riskassessment-chlorpyrifos 
Eskenazi, B., Huen, K., Marks, A., Harley, K. G., Bradman, A., Barr, D. B., \& Holland, N. (2010). PON1 and neurodevelopment in children from the CHAMACOS study exposed to organophosphate pesticides in utero. Environmental Health Perspectives, 118(12), 1775.

Eskenazi, B., Kogut, K., Huen, K., Harley, K. G., Bouchard, M., Bradman, A., ... Holland, N. (2014). Organophosphate pesticide exposure, PON1, and neurodevelopment in school-age children from the CHAMACOS study. Environmental Research, 134, 149-57. http://doi.org/10.1016/j.envres.2014.07.001

Field, A., Miles, J., \& Field, Z. (2012). Discovering Statistics Using R. Thousand Oaks, California: SAGE Publications Ltd.

Fried, P. A., Watkinson, B., \& Gray, R. (2003). Differential effects on cognitive functioning in 13to 16-year-olds prenatally exposed to cigarettes and marihuana. Neurotoxicology and Teratology, 25(4), 427-436. http://doi.org/10.1016/S0892-0362(03)00029-1

Friedman, N. P., Miyake, A., Young, S. E., Defries, J. C., Corley, R. P., \& Hewitt, J. K. (2008). Individual differences in executive functions are almost entirely genetic in origin. Journal of Experimental Psychology. General, 137(2), 201-25. http://doi.org/10.1037/00963445.137.2.201

Furlong, M. A., Engel, S. M., Barr, D. B., \& Wolff, M. S. (2014). Prenatal exposure to organophosphate pesticides and reciprocal social behavior in childhood. Environment International, 70, 125-131.

Furlong, M. A., Engel, S. M., Daniels, J. L., Engel, L., Goldman, B., \& Herring, A. (n.d.). Early life characteristics and neurodevelopmental phenotypes in the mount sinai children's environmental health center. In Prep.

Gale, C. R., Robinson, S. M., Godfrey, K. M., Law, C. M., Schlotz, W., \& O'Callaghan, F. J. (2008). Oily fish intake during pregnancy--association with lower hyperactivity but not with higher full-scale IQ in offspring. Journal of Child Psychology and Psychiatry, and Allied Disciplines, 49(10), 1061-8. http://doi.org/10.1111/j.1469-7610.2008.01908.x

Geldmacher-von Mallinckrodt, M., \& Diepgen, T. L. (1988). The human serum paraoxonasePolymorphism and specificity. Toxicological \& Environmental Chemistry, 18(2-3), 79-196. http://doi.org/10.1080/02772248809357310

Gelman, A., Carlin, J. B., Stern, H. S., Dunson, D. B., Vehtari, A., \& Rubin, D. (2013). Bayesian Data Analysis (3rd ed.). Boca Raton, Florida: CRC Press.

Geurts, H. M., Verté, S., Oosterlaan, J., Roeyers, H., \& Sergeant, J. A. (2004). How specific are executive functioning deficits in attention deficit hyperactivity disorder and autism? Journal of Child Psychology and Psychiatry, and Allied Disciplines, 45(4), 836-54. http://doi.org/10.1111/j.1469-7610.2004.00276.x

Gilbert, S., \& Grant-Webster, K. (1995). Neurobehavioral effects of developmental methylmercury exposure. Environmental Health Perspectives, 103(Suppl 6), 135-42. 
Gioia, G. A., Isquith, P. K., Guy, S. C., \& Kenworthy, L. (2000). BRIEF: Behavior rating inventory of executive function: Professional manual. Odessa, FL: Psychological Assessment Resources.

Greenberg, P. E., Sisitsky, T., Kessler, R. C., Finkelstein, S. N., Berndt, E. R., Davidson, J. R., ... Fyer, A. J. (1999). The economic burden of anxiety disorders in the 1990s. The Journal of Clinical Psychiatry, 60(7), 427-35. Retrieved from

http://www.ncbi.nlm.nih.gov/pubmed/10453795

Greenland, S. (2003). Quantifying biases in causal models: classical confounding vs colliderstratification bias. Epidemiology (Cambridge, Mass.), 14(3), 300-306. http://doi.org/10.1097/01.EDE.0000042804.12056.6C

Hashjin, G. S., Dizaj, F. S., Attaran, H., \& Koohi, M. K. (2013). Malathion induces anxiety in the male adult mouse. Archives of Medical Science: AMS, 9(2), 368-71. http://doi.org/10.5114/aoms.2013.33174

Hayton, J. C., Allen, D. G., \& Scarpello, V. (2004). Factor retention decisions in exploratory factor analysis: a tutorial on parallel analysis. Organizational Research Methods, 7(2), 191205. http://doi.org/10.1177/1094428104263675

Herring, A. H. (2010). Nonparametric Bayes shrinkage for assessing exposures to mixtures subject to limits of detection. Epidemiology (Cambridge, Mass.), 21(Suppl 4), S71.

Hibbeln, J. R., Davis, J. M., Steer, C., Emmett, P., Rogers, I., Williams, C., \& Golding, J. (2007). Maternal seafood consumption in pregnancy and neurodevelopmental outcomes in childhood (ALSPAC study): an observational cohort study. Lancet (London, England), 369(9561), 578-85. http://doi.org/10.1016/S0140-6736(07)60277-3

Indredavik, M. S., Brubakk, A.-M., Romundstad, P., \& Vik, T. (2007). Prenatal smoking exposure and psychiatric symptoms in adolescence. Acta Paediatrica (Oslo, Norway: 1992), 96(3), 377-82. http://doi.org/10.1111/j.1651-2227.2006.00148.x

Jensen, P. S., Martin, D., \& Cantwell, D. (1997). Comorbidity in ADHD: Implications for research, practice, and DSM-V. Journal of the American Academy of Child and Adolescent Psychiatry, 36(8), 1065-1079.

Johnson, S., \& Marlow, N. (2011). Preterm birth and childhood psychiatric disorders. Pediatric Research, 69(5 PART 2), 11R-18R. http://doi.org/10.1203/PDR.0b013e318212faa0

Johnson, W., \& Bouchard, T. J. (2005). The structure of human intelligence: It is verbal, perceptual, and image rotation (VPR), not fluid and crystallized. Intelligence, 33(4), 393416. http://doi.org/10.1016/j.intell.2004.12.002

Julvez, J., Ribas-Fitó, N., Torrent, M., Forns, M., Garcia-Esteban, R., \& Sunyer, J. (2007). Maternal smoking habits and cognitive development of children at age 4 years in a population-based birth cohort. International Journal of Epidemiology, 36(4), 825-32. http://doi.org/10.1093/ije/dym107 
Kato, K., Silva, M. J., Needham, L. L., \& Calafat, A. M. (2005). Determination of 16 phthalate metabolites in urine using automated sample preparation and on-line preconcentration/high-performance liquid chromatography/tandem mass spectrometry. Analytical Chemistry, 77(9), 2985-91. http://doi.org/10.1021/ac0481248

Kaufman, J. S., Maclehose, R. F., \& Kaufman, S. (2004). A further critique of the analytic strategy of adjusting for covariates to identify biologic mediation. Epidemiologic Perspectives \& Innovations : EP+I, 1(1), 4. http://doi.org/10.1186/1742-5573-1-4

Lainhart, J. E., Piven, J., Wzorek, M., Landa, R., Santangelo, S. L., Coon, H., \& Folstein, S. E. (1997). Macrocephaly in children and adults with autism. Journal of the American Academy of Child and Adolescent Psychiatry, 36(2), 282-90. http://doi.org/10.1097/00004583199702000-00019

Landrigan, P. J., Schechter, C. B., Lipton, J. M., Fahs, M. C., \& Schwartz, J. (2002). Environmental pollutants and disease in American children: estimates of morbidity, mortality, and costs for lead poisoning, asthma, cancer, and developmental disabilities. Environmental Health Perspectives, 110(7), 721.

Langley, K., Rice, F., van den Bree, M. B. M., \& Thapar, A. (2005). Maternal smoking during pregnancy as an environmental risk factor for attention deficit hyperactivity disorder behaviour. A review. Minerva Pediatrica, 57(6), 359-71. Retrieved from http://europepmc.org/abstract/med/16402008

Li, W.-F., Costa, L. G., Richter, R. J., Hagen, T., Shih, D., Tward, A., ... Furlong, C. (2000). Catalytic efficiency determines the in-vivo efficacy of PON1 for detoxifying organophosphorus compounds. Pharmacogenetics, 10(9), 767-779.

Linnet, K. M., Dalsgaard, S., Obel, C., Wisborg, K., Henriksen, T. B., Rodriguez, A., ... Jarvelin, M.-R. (2003). Maternal lifestyle factors in pregnancy risk of attention deficit hyperactivity disorder and associated behaviors: review of the current evidence. The American Journal of Psychiatry, 160(6), 1028-40. http://doi.org/10.1176/appi.ajp.160.6.1028

Lu, C., Barr, D. B., Pearson, M. A., \& Waller, L. A. (2008). Dietary intake and its contribution to longitudinal organophosphorus pesticide exposure in urban/suburban children. Environmental Health Perspectives, 116(4), 537-42. http://doi.org/10.1289/ehp.10912

MacCallum, R. S., Widaman, K. F., Zhang, S., \& Hong, S. (1999). Sample size in factor analysis. Psychological Methods, 4(1), 84-89.

MacLean, E. L., Hare, B., Nunn, C. L., Addessi, E., Amici, F., Anderson, R. C., ... Zhao, Y. (2014). The evolution of self-control. Proceedings of the National Academy of Sciences of the United States of America, 111(20), E2140-8. http://doi.org/10.1073/pnas.1323533111

MacLehose, R. F., Dunson, D. B., Herring, A. H., \& Hoppin, J. A. (2007). Bayesian methods for highly correlated exposure data. Epidemiology, 18(2), 199-207.

Marks, A. R., Harley, K., Bradman, A., Kogut, K., Barr, D. B., Johnson, C., ... Eskenazi, B. (2010). Organophosphate pesticide exposure and attention in young Mexican-American children: The CHAMACOS study. Environmental Health Perspectives, 118(12), 17681774. http://doi.org/10.1289/ehp.1002056 
Mattison, R. E., \& Mayes, S. D. (2012). Relationships Between Learning Disability, Executive Function, and Psychopathology in Children With ADHD. Journal of Attention Disorders, 16(2), 138-146. http://doi.org/10.1177/1087054710380188

McAuley, T., Chen, S., Goos, L., Schachar, R., \& Crosbie, J. (2010). Is the behavior rating inventory of executive function more strongly associated with measures of impairment or executive function? Journal of the International Neuropsychological Society: JINS, 16(3), 495-505. http://doi.org/10.1017/S1355617710000093

Middlemore-Risher, M. L., Buccafusco, J. J., \& Terry, A. V. (2010). Repeated exposures to lowlevel chlorpyrifos results in impairments in sustained attention and increased impulsivity in rats. Neurotoxicology and Teratology, 32(4), 415-424. http://doi.org/10.1016/j.ntt.2010.03.008

N'Go, P. K., Azzaoui, F.-Z., Ahami, A. O. T., Soro, P. R., Najimi, M., \& Chigr, F. (2013). Developmental effects of Malathion exposure on locomotor activity and anxiety-like behavior in Wistar rat. Health, 05(03), 603-611. http://doi.org/10.4236/health.2013.53A080

Oken, E., Wright, R. O., Kleinman, K. P., Bellinger, D., Amarasiriwardena, C. J., Hu, H., ... Gillman, M. W. (2005). Maternal fish consumption, hair mercury, and infant cognition in a U.S. cohort. Environmental Health Perspectives, 113(10), 1376-1380. http://doi.org/10.1289/ehp.8041

Piper, B. J., \& Corbett, S. M. (2012). Executive function profile in the offspring of women that smoked during pregnancy. Nicotine \& Tobacco Research : Official Journal of the Society for Research on Nicotine and Tobacco, 14(2), 191-9. http://doi.org/10.1093/ntr/ntr181

Qi, X., Zheng, M., Wu, C., Chang, X., Wang, G., Lu, D., \& Zhou, Z. (2011). Impact of prenatal pyrethroid exposure on neurodevelopment of one-year old infants. Journal of Hygiene Research, 40(6), 693-7. Retrieved from http://www.ncbi.nlm.nih.gov/pubmed/22279658

Rauh, V. A., Garfinkel, R., Perera, F. P., Andrews, H. F., Hoepner, L., Barr, D. B., ... Whyatt, R. W. (2006). Impact of prenatal chlorpyrifos exposure on neurodevelopment in the first 3 years of life among inner-city children. Pediatrics, 118(6), e1845-59. http://doi.org/10.1542/peds.2006-0338

Rauh, V., Arunajadai, S., Horton, M., Perera, F., Hoepner, L., Barr, D. B., \& Whyatt, R. (2011). Seven-year neurodevelopmental scores and prenatal exposure to chlorpyrifos, a common agricultural pesticide. Environmental Health Perspectives, 119(8), 1196-1201. http://doi.org/10.1289/ehp.1003160

Reynolds, C., \& Kamphaus, R. (1998). BASC: Behavior assessment system for chilren: Manual. American Guidance Service.

Ricceri, L., Markina, N., Valanzano, A., Fortuna, S., Cometa, M. F., Meneguz, A., \& Calamandrei, G. (2003). Developmental exposure to chlorpyrifos alters reactivity to environmental and social cues in adolescent mice. Toxicology and Applied Pharmacology, 191(3), 189-201. http://doi.org/10.1016/S0041-008X(03)00229-1 
Ricceri, L., Venerosi, A., Capone, F., Cometa, M. F., Lorenzini, P., Fortuna, S., \& Calamandrei, G. (2006). Developmental neurotoxicity of organophosphorous pesticides: Fetal and neonatal exposure to chlorpyrifos alters sex-specific behaviors at adulthood in mice. Toxicological Sciences, 93(1), 105-113. http://doi.org/10.1093/toxsci/kfl032

Ridderinkhof, K. R., \& Van Der Molen, M. W. (1997). Mental resources, processing speed, and inhibitory control: A developmental perspective. Biological Psychology, 45(1-3), 241-261. http://doi.org/10.1016/S0301-0511(96)05230-1

Robinson, P. N. (2012). Deep phenotyping for precision medicine. Human Mutation, 33(5), 777780. http://doi.org/10.1002/humu.22080

Sameroff, A. J., Seifer, R., Barocas, R., Zax, M., \& Greenspan, S. (1987). Intelligence quotient scores of 4-year-old children: social-environmental risk factors. Pediatrics, 79(3), 343-50. Retrieved from http://www.ncbi.nlm.nih.gov/pubmed/3822634

Sandoval, J., \& Echandia, A. (1994). Behavior assessment system for children. Journal of School Psychology, 32(4), 419-425. http://doi.org/10.1016/0022-4405(94)90037-X

Sartorius, N., Ustun, T. B., Lecrubier, Y., \& Wittchen, H.-U. (1996). Depression comorbid with anxiety: results from the WHO study on "psychological disorders in primary health care." The British Journal of Psychiatry, 168(Suppl 30), 38-43.

Semrud-Clikeman, M., Walkowiak, J., Wilkinson, A., \& Butcher, B. (2010). Executive functioning in children with Asperger syndrome, ADHD-combined type, ADHD-predominately inattentive type, and controls. Journal of Autism and Developmental Disorders, 40(8), 1017-1027.

Sinzig, J., Morsch, D., Bruning, N., Schmidt, M. H., \& Lehmkuhl, G. (2008). Inhibition, flexibility, working memory and planning in autism spectrum disorders with and without comorbid ADHD-symptoms. Child and Adolescent Psychiatry and Mental Health, 2(4), 1-12. http://doi.org/10.1186/1753-2000-2-4

Slotkin, T. A., \& Seidler, F. J. (2005). The alterations in CNS serotonergic mechanisms caused by neonatal chlorpyrifos exposure are permanent. Developmental Brain Research, 158(1), $115-119$.

Slotkin, T. A., \& Seidler, F. J. (2008). Developmental neurotoxicants target neurodifferentiation into the serotonin phenotype: Chlorpyrifos, diazinon, dieldrin and divalent nickel. Toxicology and Applied Pharmacology, 233(2), 211-219. http://doi.org/10.1016/j.taap.2008.08.020

Stene-Larsen, K., Borge, A. I. H., \& Vollrath, M. E. (2009). Maternal smoking in pregnancy and externalizing behavior in 18-month-old children: results from a population-based prospective study. Journal of the American Academy of Child and Adolescent Psychiatry, 48(3), 283-9. http://doi.org/10.1097/CHI.0b013e318195bcfb

Thakur, G. A., Sengupta, S. M., Grizenko, N., Choudhry, Z., \& Joober, R. (2012). Family-based association study of ADHD and genes increasing the risk for smoking behaviours. Archives of Disease in Childhood, 97(12), 1027-33. http://doi.org/10.1136/archdischild-2012-301882 
Thapar, A., Rice, F., Hay, D., Boivin, J., Langley, K., van den Bree, M., ... Harold, G. (2009). Prenatal smoking might not cause attention-deficit/hyperactivity disorder: Evidence from a novel design. Biological Psychiatry, 66(8), 722-7.

http://doi.org/10.1016/j.biopsych.2009.05.032

Toplak, M. E., West, R. F., \& Stanovich, K. E. (2013). Practitioner review: do performancebased measures and ratings of executive function assess the same construct? Journal of Child Psychology and Psychiatry, and Allied Disciplines, 54(2), 131-43. http://doi.org/10.1111/jcpp.12001

Ullman, M. T., \& Pullman, M. Y. (2015). A compensatory role for declarative memory in neurodevelopmental disorders. Neuroscience and Biobehavioral Reviews, 51, 205-22. http://doi.org/10.1016/j.neubiorev.2015.01.008

Van den Bergh, B. R. H., Mulder, E. J. H., Mennes, M., \& Glover, V. (2005). Antenatal maternal anxiety and stress and the neurobehavioural development of the fetus and child: links and possible mechanisms. A review. Neuroscience \& Biobehavioral Reviews, 29(2), 237-258. http://doi.org/10.1016/j.neubiorev.2004.10.007

Viel, J.-F., Warembourg, C., Le Maner-Idrissi, G., Lacroix, A., Limon, G., Rouget, F., ... Chevrier, C. (2015). Pyrethroid insecticide exposure and cognitive developmental disabilities in children: The PELAGIE mother-child cohort. Environment International, 82, 69-75. http://doi.org/10.1016/j.envint.2015.05.009

Watkins, D. J., Fortenberry, G. Z., Sánchez, B. N., Barr, D. B., Panuwet, P., Schnaas, L., ... Meeker, J. D. (2016). Urinary 3-phenoxybenzoic acid (3-PBA) levels among pregnant women in Mexico City: Distribution and relationships with child neurodevelopment. Environmental Research, 147, 307-13. http://doi.org/10.1016/j.envres.2016.02.025

Wessels, D., Barr, D. B., \& Mendola, P. (2003). Use of biomarkers to indicate exposure of children to organophosphate pesticides: implications for a longitudinal study of children's environmental health. Environmental Health Perspectives, 111(16), 1939.

Whyatt, R. M., Garfinkel, R., Hoepner, L. A., Andrews, H., Holmes, D., Williams, M. K., ... Barr, D. B. (2009). A Biomarker Validation Study of Prenatal Chlorpyrifos Exposure within an Inner-City Cohort during Pregnancy. Environmental Health Perspectives, 117(4), 559-567. http://doi.org/10.1289/ehp.0800041

Willford, J., Leech, S., \& Day, N. (2006). Moderate prenatal alcohol exposure and cognitive status of children at age 10. Alcoholism, Clinical and Experimental Research, 30(6), 10519. http://doi.org/10.1111/j.1530-0277.2006.00119.x

Williams, G. M., O’Callaghan, M., Najman, J. M., Bor, W., Andersen, F. M. J., Richards, A. D., \& BSc, C. U. (1998). Maternal Cigarette Smoking and Child Psychiatric Morbidity: A Longitudinal Study. Pediatrics, 102(1), e11-. Retrieved from http://pediatrics.aappublications.org.libproxy.lib.unc.edu/content/102/1/e11.short

Wolff, M. S., Engel, S., Berkowitz, G., Teitelbaum, S., Siskind, J., Barr, D. B., \& Wetmur, J. (2007). Prenatal pesticide and PCB exposures and birth outcomes. Pediatric Research, 61(2), 243-50. http://doi.org/10.1203/pdr.0b013e31802d77f0 
Wolff, M. S., Engel, S. M., Berkowitz, G. S., Ye, X., Silva, M. J., Zhu, C., ... Calafat, A. M. (2008). Prenatal phenol and phthalate exposures and birth outcomes. Environmental Health Perspectives, 116(8), 1092-1097. http://doi.org/10.1289/ehp.11007

Woodhouse, W., Bailey, a, Rutter, M., Bolton, P., Baird, G., \& Le Couteur, a. (1996). Head circumference in autism and other pervasive developmental disorders. Journal of Child Psychology and Psychiatry, and Allied Disciplines, 37(6), 665-71. http://doi.org/10.1111/j.1469-7610.1996.tb01458.x

Ye, X., Kuklenyik, Z., Needham, L. L., \& Calafat, A. M. (2005). Quantification of urinary conjugates of bisphenol A, 2,5-dichlorophenol, and 2-hydroxy-4-methoxybenzophenone in humans by online solid phase extraction-high performance liquid chromatography-tandem mass spectrometry. Analytical and Bioanalytical Chemistry, 383(4), 638-44. http://doi.org/10.1007/s00216-005-0019-4

Yu, C.-J., Du, J.-C., Chiou, H.-C., Chung, M.-Y., Yang, W., Chen, Y.-S., ... Chen, M.-L. (2016). Increased risk of attention-deficit/hyperactivity disorder associated with exposure to organophosphate pesticide in Taiwanese children. Andrology. http://doi.org/10.1111/andr.12183

Zhang, X., Driver, J. H., Li, Y., Ross, J. H., \& Krieger, R. I. (2008). Dialkylphosphates (DAPs) in fruits and vegetables may confound biomonitoring in organophosphorus insecticide exposure and risk assessment. Journal of Agricultural and Food Chemistry, 56(22), 1063810645. http://doi.org/10.1021/jf8018084

Zoroğlu, S. S., Erdal, M. E., Alaşehirli, B., Erdal, N., Sivasli, E., Tutkun, H., ... Herken, H. (2002). Significance of serotonin transporter gene 5-HTTLPR and variable number of tandem repeat polymorphism in attention deficit hyperactivity disorder.

Neuropsychobiology, 45(4), 176-181. 